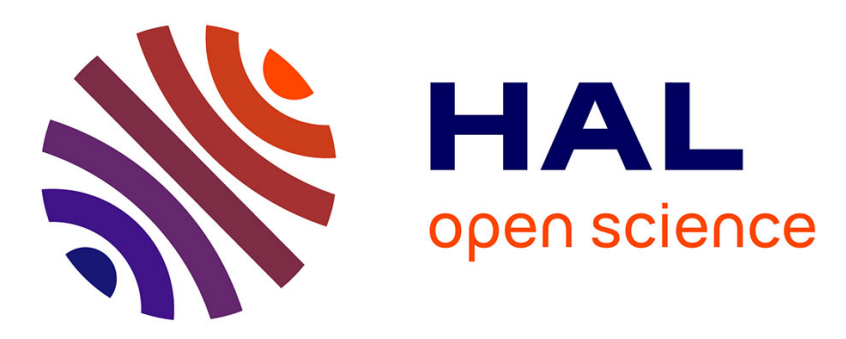

\title{
Mathematical analysis of a three-tiered model of anaerobic digestion
}

Sarra Nouaoura, Nahla Abdellatif, Radhouane Fekih-Salem, Tewfik Sari

\section{To cite this version:}

Sarra Nouaoura, Nahla Abdellatif, Radhouane Fekih-Salem, Tewfik Sari. Mathematical analysis of a three-tiered model of anaerobic digestion. SIAM Journal on Applied Mathematics, 2021, 81 ((3)), pp.1264-1286. 10.1137/20M1353897 . hal-02540350v3

\section{HAL Id: hal-02540350 \\ https://hal.science/hal-02540350v3}

Submitted on 12 Jan 2021

HAL is a multi-disciplinary open access archive for the deposit and dissemination of scientific research documents, whether they are published or not. The documents may come from teaching and research institutions in France or abroad, or from public or private research centers.
L'archive ouverte pluridisciplinaire HAL, est destinée au dépôt et à la diffusion de documents scientifiques de niveau recherche, publiés ou non, émanant des établissements d'enseignement et de recherche français ou étrangers, des laboratoires publics ou privés. 


\title{
MATHEMATICAL ANALYSIS OF A THREE-TIERED MODEL OF ANAEROBIC DIGESTION *
}

\author{
SARRA NOUAOURA ${ }^{\dagger}$, NAHLA ABDELLATIF ${ }^{\dagger \ddagger}$, RADHOUANE FEKIH-SALEM $^{\dagger} \S$, AND \\ TEWFIK SARI 9
}

\begin{abstract}
In this paper, we are interested in a mechanistic model describing the anaerobic mineralization of chlorophenol in a three-step food-web. The model is a six-dimensional system of ordinary differential equations. In our study, we take into account the phenol and the hydrogen input concentrations as well as the maintenance terms. Moreover, we consider the case of a large class of growth rates, instead of specific kinetics. In this general case, a recent study shows that the system can have up to eight steady states and their existence conditions were analytically determined. We focus here on the necessary and sufficient conditions of the local stability of the steady states, according to the four operating parameters of the process, which are the dilution rate and the chlorophenol, phenol and hydrogen input concentrations. In previous studies, this stability analysis was performed only numerically. Using the Liénard-Chipart stability criterion, we show that the positive steady state can be unstable and we give numerical evidence for a supercritical Hopf bifurcation with the appearance of a stable periodic orbit. We give two bifurcation diagrams with respect to the dilution rate, first, and then to the chlorophenol input concentration as the bifurcating parameters, showing that the system can present rich behavior including bistability, coexistence and occurrence of limit cycle.
\end{abstract}

Key words. Anaerobic digestion, Chemostat, Chlorophenol mineralization, Hopf bifurcation, Liénard-Chipart stability criterion, Limit cycle.

AMS subject classifications. 34A34, 34D20, 37N25, 92B05

1. Introduction. Anaerobic digestion is a natural process in which organic material is converted into biogas in an environment without oxygen by the action of a microbial ecosystem. It is used for the treatment of wastewater and organic solid wastes and has the advantage of producing methane and hydrogen under appropriate conditions [13]. The removed carbon dioxide can be used too as a carbon source for microalgae [12]. It is used also for several industrial or domestic purposes in biorefineries and other anaerobic technologies. For a recent review on the current state-of-the-art with respect to the theory, applications, and technologies, the reader is referred to Wade [25].

The full Anaerobic Digestion Model No.1.(ADM1) [1] is highly parameterized with a large number of state variables. Whilst suitable for dynamic simulation, analytical results on the model are impossible and only numerical investigations are available [4]. Due to the analytical intractability of the full ADM1, simpler mechanistic models of microbial interaction have been proposed in view of a better understanding of the anaerobic digestion process.

The two-tiered models, which take the form of four-dimensional mathematical models with a cascade of two biological reactions, where one substrate is consumed

\footnotetext{
* Submitted to the editors 2020-07-17.
}

Funding: This work was supported by the Euro-Mediterranean research network TREASURE (http://www.inra.fr/treasure).

${ }^{\dagger}$ University of Tunis El Manar, National Engineering School of Tunis, LAMSIN, 1002, Tunis, Tunisia (sarra.nouaoura@enit.utm.tn).

¥University of Manouba, National School of Computer Science, 2010, Manouba, Tunisia (nahla.abdellatif@ensi-uma.tn).

$\S$ University of Monastir, Higher Institute of Computer Science of Mahdia, 5111, Mahdia, Tunisia (radhouene.fekihsalem@isima.rnu.tn).

ฯ ITAP, Univ Montpellier, INRAE, Institut Agro, Montpellier, France (tewfik.sari@inrae.fr). 
by one microorganism in a chemostat to produce a product that serves as the main limiting substrate for a second microorganism, are the simplest models which encapsulate the essence of the anaerobic digestion process. Two-tiered models with commensalistic relationship including or not substrate inhibition of the second population are widely considered $[2,3,17,21]$ where the second population (the commensal population) benefits for its growth from the first population (the host population) while the host population is not affected by the growth of the commensal population. On the contrary, when the growth of the first population is affected by the growth of the second population, the system describes a syntrophic relationship [5, 7, 9, 18, 19, 24, 29]. For more details and informations on commensalism and syntrophy, the reader is referred to [19] and the references therein. Important and interesting extensions of the two-tiered models are the eight-dimensional mathematical models, which include syntrophy and inhibition $[27,28]$ and the model with five state variables studied in $[4,15]$.

In this paper, we consider a six-dimensional mathematical model, which is an extension, with generalized growth functions, of the three-tiered food-web studied by Wade et al. [26]. For a description of this food-web, where the microorganisms involved are chlorophenol and phenol degraders and hydrogenotrophic methanogen, see section 4. Note that the three-tiered food-web is not a classical anaerobic digestion process since the chlorophenol mineralization may occur under aerobic or anaerobic conditions with different microbial consortia involved. For more details on the biological significance of this food-web and its relation to the complete ADM1, the reader is invited to refer to [26]. It has been shown in [26] that this model can have up to eight steady states. Arguing that the Routh-Hurwitz theorem allowing for an explicit analysis of the stability of steady states, is intractable beyond five dimensions, as it was noticed in [14], the stability of the steady states were determined only numerically [26] using specific growth rates (see formulas (4.1)). Several operating diagrams, which are the bifurcation diagrams with respect to the four operating parameters (i.e. the dilution rate, the chlorophenol, the phenol and the hydrogen input concentrations) have been numerically constructed in [26], showing the role, and the importance of each operating parameter, in particular for the coexistence of all three species.

The model of [26] is extended in [16, 20] with general growth rates (see section 2 for the assumptions on the growth rates) and takes the form:

$$
\left\{\begin{array}{l}
\dot{x}_{0}=\left(\mu_{0}\left(s_{0}, s_{2}\right)-D-a_{0}\right) x_{0} \\
\dot{x}_{1}=\left(\mu_{1}\left(s_{1}, s_{2}\right)-D-a_{1}\right) x_{1} \\
\dot{x}_{2}=\left(\mu_{2}\left(s_{2}\right)-D-a_{2}\right) x_{2} \\
\dot{s}_{0}=D\left(s_{0}^{\text {in }}-s_{0}\right)-\mu_{0}\left(s_{0}, s_{2}\right) x_{0} \\
\dot{s}_{1}=D\left(s_{1}^{\text {in }}-s_{1}\right)+\mu_{0}\left(s_{0}, s_{2}\right) x_{0}-\mu_{1}\left(s_{1}, s_{2}\right) x_{1} \\
\dot{s}_{2}=D\left(s_{2}^{\text {in }}-s_{2}\right)-\omega \mu_{0}\left(s_{0}, s_{2}\right) x_{0}+\mu_{1}\left(s_{1}, s_{2}\right) x_{1}-\mu_{2}\left(s_{2}\right) x_{2}
\end{array}\right.
$$

where $s_{0}, s_{1}$ and $s_{2}$ are the substrate concentrations (chlorophenol, phenol and hydrogen, in the application); $x_{0}, x_{1}$ and $x_{2}$ are the biomass concentrations; $D$ is the dilution rate; $\mu_{i}$ is the specific growth rate; $s_{i}^{\text {in }}$ is the input substrate concentration in the chemostat; $\omega$ is a yield coefficient; $a_{i}$ is the maintenance (or decay) rate for $i=0,1,2$ and corresponding to chlorophenol, phenol and hydrogen, respectively. As explained in [26], the chlorophenol degrader grows on both chlorophenol and hydrogen and produces phenol. The phenol degrader consumes the phenol to form hydrogen, which inhibits its growth. The hydrogenotrophic methanogen grows on the produced hydrogen. 
The mathematical analysis of (1.1), under various assumptions, is given in $[8,16$, $20,23]$. The system (1.1) was studied in [20] in the case $s_{0}^{\text {in }}>0$ and $s_{1}^{\text {in }}=s_{2}^{\text {in }}=0$ where at most three types of steady states can exist. The necessary and sufficient conditions of existence of the steady states are analytically determined, showing their uniqueness, except for one of them, that can exist in two forms. When maintenance is neglected ( $a_{0}, a_{1}$ and $a_{2}$ are assumed to be zero), the six-dimensional mathematical model can be reduced to a three-dimensional one and the stability of steady states was analytically characterized. It has been also shown in [20] that the positive steady state can be unstable, a fact that has not been described in [26]. Numerical analysis has suggested the presence of a Hopf bifurcation emerging through the positive steady state, with the chlorophenol input concentration as the bifurcating parameter. System (1.1) was studied in [23] in the case without maintenance and persistence results were analytically proved. Using numerical estimation, it is shown in [23] that the system has a rich dynamics including Hopf, Bogdanov-Takens and Bautin bifurcations. The three-tiered model of [26] was simplified in [8] by neglecting the part of hydrogen produced by the phenol degrader $\left(\mu_{1}\left(s_{1}, s_{2}\right) x_{1}\right.$ is not considered in the model) as well as maintenance, which gives rise to a less realistic model. However, the existence and stability of steady states were analytically studied and a global analysis is performed, proving the asymptotic persistence of the three bacteria. The results of [20] were extended in [16] in the case $s_{1}^{\text {in }} \geq 0$ and $s_{2}^{\text {in }} \geq 0$. When the inflow of the three substrates is included, the system can have at most eight types of steady states. The necessary and sufficient conditions of existence of the steady states are analytically determined when maintenance is included. The necessary and sufficient conditions of stability are analytically determined only when maintenance is neglected.

Here, we focus on the analysis of the stability of all steady states of (1.1), and we analytically characterize the stability, by using the Liénard-Chipart stability criterion, in the case including maintenance, where the system cannot be reduced to a threedimensional one. We then generalize [26] by allowing a larger class of growth functions and by giving rigorous proofs for the results on the existence and stability of steady states. For this class of growth functions, we generalize [8, 16, 20, 23] by giving the necessary and sufficient conditions of stability of steady states when maintenance is included in the model.

This paper is organized as follows: in section 2, we recall the general assumptions on the growth functions and the steady states of model (1.1). We give in section 3 the necessary and sufficient conditions of existence and stability of the steady states. Next, in section 4, we give an application of our theoretical results to the threetiered model considered in [26]. We dedicate section 5 to discuss our results. In Appendix A, we define some auxiliary functions used for the description of the steady states with their conditions of existence and stability. The Liénard-Chipart stability criterion and all the proofs are reported in Appendices B and C, respectively. In Appendix D, the description of the bifurcation diagram according to the dilution rate is supported by numerical experimentation. The bifurcation diagram according to the chlorophenol input concentration is determined in Appendix $\mathrm{E}$ and it is supported by numerical experimentation in Appendix F. Details and complements on the threetiered model considered in [26] are given in Appendix G. In Appendix H, we illustrate some numerical simulations and some tables are given in Appendix I.

2. Assumptions and steady states. We consider model (1.1). Following [16, 20], we assume that the growth functions are continuously differentiable $\left(\mathcal{C}^{1}\right)$ and satisfy the following conditions: 
(H1) For all $s_{0}>0$ and $s_{2}>0,0<\mu_{0}\left(s_{0}, s_{2}\right)<+\infty, \mu_{0}\left(0, s_{2}\right)=0, \mu_{0}\left(s_{0}, 0\right)=0$.

(H2) For all $s_{1}>0$ and $s_{2} \geq 0,0<\mu_{1}\left(s_{1}, s_{2}\right)<+\infty, \mu_{1}\left(0, s_{2}\right)=0$.

(H3) For all $s_{2}>0,0<\mu_{2}\left(s_{2}\right)<+\infty, \mu_{2}(0)=0$.

(H4) For all $s_{0}>0$ and $s_{2}>0, \frac{\partial \mu_{0}}{\partial s_{0}}\left(s_{0}, s_{2}\right)>0, \frac{\partial \mu_{0}}{\partial s_{2}}\left(s_{0}, s_{2}\right)>0$.

(H5) For all $s_{1}>0$ and $s_{2}>0, \frac{\partial \mu_{1}}{\partial s_{1}}\left(s_{1}, s_{2}\right)>0, \quad \frac{\partial \mu_{1}}{\partial s_{2}}\left(s_{1}, s_{2}\right)<0$.

(H6) For all $s_{2}>0, \mu_{2}^{\prime}\left(s_{2}\right)>0$.

(H7) The function $s_{2} \mapsto \mu_{0}\left(+\infty, s_{2}\right)$ is monotonically increasing and the function $s_{2} \mapsto \mu_{1}\left(+\infty, s_{2}\right)$ is monotonically decreasing.

Let $\Psi$ the function defined in Table 8 of Appendix A. Then, we assume that:

(H8) When $\omega<1$, the function $\Psi$ has a unique minimum $\bar{s}_{2}=\bar{s}_{2}(D)$ on the interval $\left(s_{2}^{0}, s_{2}^{1}\right)$, such that $\frac{\partial \Psi}{\partial s_{2}}\left(s_{2}, D\right)<0$ on $\left(s_{2}^{0}, \bar{s}_{2}\right)$ and $\frac{\partial \Psi}{\partial s_{2}}\left(s_{2}, D\right)>0$ on $\left(\bar{s}_{2}, s_{2}^{1}\right)$.

All other auxiliary functions needed in the afterward results are provided in Appendix A. Under Hypotheses (H1) to (H6), system (1.1) can have at most eight types of steady states whose components are given in Table 1, see Theorem 1 in [16]. Notice that a steady state exists or is said to be 'meaningful' if and only if all its components are nonnegative.

TABLE 1

Steady states of (1.1). All functions are defined in Table 8.

$s_{0}, s_{1}, s_{2}$ and $x_{0}, x_{1}, x_{2}$ components

\begin{tabular}{|c|c|}
\hline 51 & $s_{0}=s_{0}^{\text {in }}, s_{1}=s_{1}^{\text {in }}, s_{2}=s_{2}^{\text {in }}$ and $x_{0}=0, x_{1}=0, x_{2}=0$ \\
\hline $\mathrm{SS} 2$ & $s_{0}=s_{0}^{\text {in }}, s_{1}=s_{1}^{\text {in }}, s_{2}=M_{2}\left(D+a_{2}\right)$ and $x_{0}=0, x_{1}=0, x_{2}=\frac{D}{D+a_{2}}\left(s_{2}^{\text {in }}-s_{2}\right)$ \\
\hline SS3 & $\begin{array}{l}s_{1}=s_{1}^{\text {in }}+s_{0}^{\text {in }}-s_{0} \text { and } s_{2}=s_{2}^{\text {in }}-\omega\left(s_{0}^{\text {in }}-s_{0}\right), \text { where } s_{0} \text { is a solution of } \\
\psi_{0}\left(s_{0}\right)=D+a_{0} \text { and } x_{0}=\frac{D}{D+a_{0}}\left(s_{0}^{\text {in }}-s_{0}\right), x_{1}=0, x_{2}=0\end{array}$ \\
\hline $\mathrm{SS} 4$ & $\begin{array}{l}s_{0}=M_{0}\left(D+a_{0}, s_{2}\right) \text { and } s_{1}=M_{1}\left(D+a_{1}, s_{2}\right), \text { where } s_{2} \text { is a solution of } \\
\Psi\left(s_{2}, D\right)=(1-\omega) s_{0}^{\text {in }}+s_{1}^{\text {in }}+s_{2}^{\text {in }} \\
\text { and } x_{0}=\frac{D}{D+a_{0}}\left(s_{0}^{\text {in }}-s_{0}\right), x_{1}=\frac{D}{D+a_{1}}\left(s_{0}^{\text {in }}-s_{0}+s_{1}^{\text {in }}-s_{1}\right), x_{2}=0\end{array}$ \\
\hline SS5 & $\begin{array}{l}s_{0}=\varphi_{0}(D), s_{1}=s_{1}^{\text {in }}+s_{0}^{\text {in }}-s_{0}, s_{2}=M_{2}\left(D+a_{2}\right) \\
\text { and } x_{0}=\frac{D}{D+a_{0}}\left(s_{0}^{\text {in }}-s_{0}\right), x_{1}=0, x_{2}=\frac{D}{D+a_{2}}\left(s_{2}^{\text {in }}-s_{2}-\omega\left(s_{0}^{\text {in }}-s_{0}\right)\right)\end{array}$ \\
\hline SS6 & $\begin{array}{l}s_{0}=\varphi_{0}(D), s_{1}=\varphi_{1}(D), s_{2}=M_{2}\left(D+a_{2}\right) \text { and } x_{0}=\frac{D}{D+a_{0}}\left(s_{0}^{\text {in }}-s_{0}\right), \\
x_{1}=\frac{D}{D+a_{1}}\left(s_{0}^{\text {in }}-s_{0}+s_{1}^{\text {in }}-s_{1}\right), x_{2}=\frac{D}{D+a_{2}}\left((1-\omega)\left(s_{0}^{\text {in }}-s_{0}\right)+s_{1}^{\text {in }}-s_{1}+s_{2}^{\text {in }}-s_{2}\right)\end{array}$ \\
\hline SS7 & $\begin{array}{l}s_{0}=s_{0}^{\text {in }} \text { and } s_{2}=s_{2}^{\text {in }}+s_{1}^{\text {in }}-s_{1}, \text { where } s_{1} \text { is a solution of } \psi_{1}\left(s_{1}\right)=D+a_{1} \\
\text { and } x_{0}=0, x_{1}=\frac{D}{D+a_{1}}\left(s_{1}^{\text {in }}-s_{1}\right), x_{2}=0\end{array}$ \\
\hline 8 & $\begin{array}{l}s_{0}=s_{0}^{\text {in }}, s_{1}=\varphi_{1}(D), s_{2}=M_{2}\left(D+a_{2}\right) \\
\text { and } x_{0}=0, x_{1}=\frac{D}{D+a_{1}}\left(s_{1}^{\text {in }}-s_{1}\right), x_{2}=\frac{D}{D+a_{2}}\left(s_{1}^{\text {in }}-s_{1}+s_{2}^{\text {in }}\right.\end{array}$ \\
\hline
\end{tabular}

3. Mathematical analysis. In this section, the necessary and sufficient conditions of existence and stability of all steady states are given in Table 3. Any reference to steady state stability should be considered as local exponential stability, that is to say, the real parts of the eigenvalues of the Jacobian matrix are negative. We need the following notations:

$$
\begin{aligned}
& E=\frac{\partial \mu_{0}}{\partial s_{0}}\left(s_{0}, s_{2}\right), \quad F=\frac{\partial \mu_{0}}{\partial s_{2}}\left(s_{0}, s_{2}\right), \quad G=\frac{\partial \mu_{1}}{\partial s_{1}}\left(s_{1}, s_{2}\right), \quad H=-\frac{\partial \mu_{1}}{\partial s_{2}}\left(s_{1}, s_{2}\right), \\
& I=\mu_{2}^{\prime}\left(s_{2}\right), \quad J=\mu_{0}\left(s_{0}, s_{2}\right), \quad K=\mu_{1}\left(s_{1}, s_{2}\right), \quad L=\mu_{2}\left(s_{2}\right) .
\end{aligned}
$$

We have used the opposite sign of the partial derivative $H=-\partial \mu_{1} / \partial s_{2}$, such that all constants involved in the computation become positive. Using the Liénard-Chipart stability criterion, the asymptotic stability of SS6 requires definitions and notations that are given in Table 2. Now, we can state our main result. 
TABLE 2

Liénard-Chipart coefficients for SS6. The functions $E, F, G, H, I, J, K$ and $L$, defined by (3.1), are evaluated at the components of SS6 given in Table 1. Notice that they are depending on the operating parameter D.

$$
\begin{aligned}
c_{1}= & 3 D+(E+F w) x_{0}+(G+H) x_{1}+I x_{2} \\
c_{2}= & 3 D^{2}+(2 D+J)(E+\omega F) x_{0}+(2 D+K)(G+H) x_{1}+E I x_{0} x_{2}+G I x_{1} x_{2} \\
& +(2 D+L) I x_{2}+(E(G+H)-(1-\omega) F G) x_{0} x_{1} \\
c_{3}= & D^{3}+D(D+2 J)(E+\omega F) x_{0}+D(D+2 K)(G+H) x_{1}+D(D+2 L) I x_{2} \\
& +E I(D+J+L) x_{0} x_{2}+G I(D+K+L) x_{1} x_{2}+E G I x_{0} x_{1} x_{2}+(E(G+H) \\
& -(1-\omega) F G)(D+J+K) x_{0} x_{1} \\
c_{4}= & D^{2}(E+\omega F) J x_{0}+D^{2}(G+H) K x_{1}+D^{2} I L x_{2}+E I(D J+D L+J L) x_{0} x_{2} \\
& +G I(D K+D L+K L) x_{1} x_{2}+E G I(J+K+L) x_{0} x_{1} x_{2}+(E(G+H) \\
& -(1-\omega) F G)(D J+D K+J K) x_{0} x_{1} \\
c_{5}= & D E I J L x_{0} x_{2}+D G I K L x_{1} x_{2}+D(E(G+H)-(1-\omega) F G) J K x_{0} x_{1} \\
& +E G I(J K+J L+K L) x_{0} x_{1} x_{2} \\
c_{6}= & E G I J K L x_{0} x_{1} x_{2} \\
\hline r_{0}= & c_{1} c_{2}-c_{3}, \quad r_{1}=c_{1} c_{4}-c_{5}, \quad r_{2}=c_{3} r_{0}-c_{1} r_{1}, \quad r_{3}=c_{5} r_{0}-c_{1}^{2} c_{6} \\
r_{4}= & r_{1} r_{2}-r_{0} r_{3}, \quad r_{5}=r_{3} r_{4}-c_{1} c_{6} r_{2}^{2}
\end{aligned}
$$

Theorem 3.1. Assume that Hypotheses (H1) to (H8) hold. The necessary and sufficient conditions of existence and local stability of the steady states are given in Table 3 .

Remark 3.2. Let's recall that in [16] all steady states, except SS4, are unique.

- If $\omega \geq 1$, when it exists, SS4 is unique. Its stability condition $\frac{\partial \Psi}{\partial s_{2}}\left(s_{2}, D\right)>0$ is always satisfied.

- If $\omega<1$, assuming also that (H8) holds, and if $(1-\omega) s_{0}^{\text {in }}+s_{1}^{\text {in }}+s_{2}^{\text {in }}>\phi_{1}(D)$, the equation defining $s_{2}$ in Table 1 has two solutions $s_{2}^{* 1}<s_{2}^{* 2}$, such that $\frac{\partial \Psi}{\partial s_{2}}\left(s_{2}^{* 1}, D\right)<$ 0 and $\frac{\partial \Psi}{\partial s_{2}}\left(s_{2}^{* 2}, D\right)>0$. We denote by SS4 ${ }^{1}$ the steady state of type SS4 corresponding to $s_{2}^{* 1}$ while $\mathrm{SS} 4^{2}$ corresponds to $s_{2}^{* 2}$. When it exists, $\mathrm{SS} 4^{1}$ is unstable. When $\mathrm{SS} 4^{2}$ exists, its stability condition $\frac{\partial \Psi}{\partial s_{2}}\left(s_{2}, D\right)>0$ is always satisfied.

- The comparison with Table 4 of [16] shows that, with the exception of SS6, the stability conditions of the steady states are the same as in the maintenance-free case. Indeed, by replacing in the stability conditions of $\operatorname{SS} j, j=1, \ldots, 8, j \neq 6$, in Table 3 the maintenance terms $a_{i}$ by zero, for $i=0,1,2$, we find the conditions given in Table 4 of [16]. Therefore, the maintenance does not destabilize these steady states. Only their regions of existence and stability, with respect to the operating parameters, can be slightly modified when maintenance is included in the model.

From Table 3, we can deduce the following result.

Proposition 3.3.

- If SS2 or SS3 or SS7 exists then, SS1 is unstable.

- If SS6 exists then, SS2, SS4, SS5 and SS8 are unstable, when they exist.

- If SS5 exists then, SS2, SS3 and SS8 are unstable, when they exist.

- If SS8 exists then, SS7 is unstable, when it exists.

4. Applications to a three-tiered microbial 'food web'. In this section, we consider the model of a chlorophenol-mineralising three-tiered microbial 'food web' in a chemostat as application of our mathematical analysis, in order to compare our findings to the numerical results in [26]. Let $S_{\mathrm{ch}}, S_{\mathrm{ph}}$ and $S_{\mathrm{H}_{2}}$ be the chlorophenol, 
TABLE 3

Existence and stability conditions of steady states of (1.1). The functions $c_{3}, c_{5}, r_{4}$ and $r_{5}$ are defined in Table 2. All other functions are given in Table 8.

\begin{tabular}{|c|c|c|}
\hline & Existence conditions & Stability conditions \\
\hline SS1 & Always exists & $\begin{array}{l}\mu_{0}\left(s_{0}^{\text {in }}, s_{2}^{\text {in }}\right)<D+a_{0}, \mu_{1}\left(s_{1}^{\text {in }}, s_{2}^{\text {in }}\right)<D+a_{1} \\
\mu_{2}\left(s_{2}^{\text {in }}\right)<D+a_{2}\end{array}$ \\
\hline SS2 & $\mu_{2}\left(s_{2}^{\text {in }}\right)>D+a_{2}$ & $s_{0}^{\text {in }}<\varphi_{0}(D), s_{1}^{\text {in }}<\varphi_{1}(D)$ \\
\hline SS3 & $\mu_{0}\left(s_{0}^{\text {in }}, s_{2}^{\text {in }}\right)>D+a_{0}$ & $\begin{array}{l}\mu_{1}\left(s_{0}^{\text {in }}+s_{1}^{\text {in }}-s_{0}, s_{2}^{\text {in }}-\omega\left(s_{0}^{\text {in }}-s_{0}\right)\right)<D+a_{1}, \\
s_{2}^{\text {in }}-\omega s_{0}^{\text {in }}<M_{2}\left(D+a_{2}\right)-\omega \varphi_{0}(D) \\
\text { with } s_{0} \text { solution of } \psi_{0}\left(s_{0}\right)=D+a_{0}\end{array}$ \\
\hline SS4 & $\begin{array}{l}(1-\omega) s_{0}^{\text {in }}+s_{1}^{\text {in }}+s_{2}^{\text {in }} \geq \phi_{1}(D), \\
s_{0}^{\text {in }}>M_{0}\left(D+a_{0}, s_{2}\right), \\
s_{0}^{\text {in }}+s_{1}^{\text {in }}>M_{0}\left(D+a_{0}, s_{2}\right) \\
\quad+M_{1}\left(D+a_{1}, s_{2}\right) \\
\quad \begin{array}{l}\text { with } s_{2} \text { solution of equation } \\
\Psi\left(s_{2}, D\right)=(1-\omega) s_{0}^{\text {in }}+s_{1}^{\text {in }}+s_{2}^{\text {in }}\end{array}\end{array}$ & $\begin{array}{l}(1-\omega) s_{0}^{\mathrm{in}}+s_{1}^{\mathrm{in}}+s_{2}^{\mathrm{in}}<\phi_{2}(D) \\
\phi_{3}(D)>0, \frac{\partial \Psi}{\partial s_{2}}\left(s_{2}, D\right)>0\end{array}$ \\
\hline SS5 & $\begin{array}{l}s_{0}^{\text {in }}>\varphi_{0}(D) \\
s_{2}^{\text {in }}-\omega s_{0}^{\text {in }}>M_{2}\left(D+a_{2}\right)-\omega \varphi_{0}(D)\end{array}$ & $s_{0}^{\text {in }}+s_{1}^{\text {in }}<\varphi_{0}(D)+\varphi_{1}(D)$ \\
\hline SS6 & $\begin{array}{l}(1-\omega) s_{0}^{\text {in }}+s_{1}^{\text {in }}+s_{2}^{\text {in }}>\phi_{2}(D) \\
s_{0}^{\text {in }}>\varphi_{0}(D) \\
s_{0}^{\text {in }}+s_{1}^{\text {in }}>\varphi_{0}(D)+\varphi_{1}(D)\end{array}$ & $c_{3}>0, c_{5}>0, r_{4}>0, r_{5}>0$ \\
\hline SS7 & $\mu_{1}\left(s_{1}^{\text {in }}, s_{2}^{\text {in }}\right)>D+a_{1}$ & $\begin{aligned} s_{1}^{\text {in }}+s_{2}^{\text {in }} & <M_{3}\left(s_{0}^{\text {in }}, D+a_{0}\right) \\
& +M_{1}\left(D+a_{1}, M_{3}\left(s_{0}^{\text {in }}, D+a_{0}\right)\right), \\
s_{1}^{\text {in }}+s_{2}^{\text {in }} & <M_{2}\left(D+a_{2}\right)+\varphi_{1}(D)\end{aligned}$ \\
\hline SS8 & $\begin{array}{l}s_{1}^{\text {in }}>\varphi_{1}(D) \\
s_{1}^{\text {in }}+s_{2}^{\text {in }}>\varphi_{1}(D)+M_{2}\left(D+a_{2}\right)\end{array}$ & $s_{0}^{\text {in }}<\varphi_{0}(D)$ \\
\hline
\end{tabular}

phenol and hydrogen substrates concentrations. The specific growth rates take the form:

$$
\begin{aligned}
& f_{0}\left(S_{\mathrm{ch}}, S_{\mathrm{H}_{2}}\right)=\frac{k_{m, \mathrm{ch}} S_{\mathrm{ch}}}{K_{S, c h}+S_{\mathrm{ch}}} \frac{S_{\mathrm{H}_{2}}}{K_{S, \mathrm{H}_{2}, \mathrm{c}}+S_{\mathrm{H}_{2}}}, \\
& f_{1}\left(S_{\mathrm{ph}}, S_{\mathrm{H}_{2}}\right)=\frac{k_{m, \mathrm{ph}} S_{\mathrm{ph}}}{K_{S, \mathrm{ph}}+S_{\mathrm{ph}}} \frac{1}{1+S_{\mathrm{H}_{2}} / K_{I, \mathrm{H}_{2}}}, \quad f_{2}\left(S_{\mathrm{H}_{2}}\right)=\frac{k_{m, \mathrm{H}_{2}} S_{\mathrm{H}_{2}}}{K_{S, \mathrm{H}_{2}}+S_{\mathrm{H}_{2}}} .
\end{aligned}
$$

Let $X_{\mathrm{ch}}, X_{\mathrm{ph}}$ and $X_{\mathrm{H}_{2}}$ be the chlorophenol, phenol and hydrogen degrader concentrations; $S_{\mathrm{ch}}^{\mathrm{in}}, S_{\mathrm{ph}}^{\mathrm{in}}$ and $S_{\mathrm{H}_{2}}^{\mathrm{in}}$ be the input concentrations; $k_{\mathrm{dec}, \mathrm{ch}}, k_{\mathrm{dec}, \mathrm{ph}}$ and $k_{\mathrm{dec}, \mathrm{H}_{2}}$ be the decay rates. This model in [26] is described by the following system of differential equations

$$
\left\{\begin{aligned}
\dot{X}_{\mathrm{ch}}= & \left(Y_{\mathrm{ch}} f_{0}\left(S_{\mathrm{ch}}, S_{\mathrm{H}_{2}}\right)-D-k_{\mathrm{dec}, \mathrm{ch}}\right) X_{\mathrm{ch}} \\
\dot{X}_{\mathrm{ph}}= & \left(Y_{\mathrm{ph}} f_{1}\left(S_{\mathrm{ph}}, S_{\mathrm{H}_{2}}\right)-D-k_{\mathrm{dec}, \mathrm{ph}}\right) X_{\mathrm{ph}} \\
\dot{X}_{\mathrm{H}_{2}}= & \left(Y_{\mathrm{H}_{2}} f_{2}\left(S_{\mathrm{H}_{2}}\right)-D-k_{\mathrm{dec}, \mathrm{H}_{2}}\right) X_{\mathrm{H}_{2}} \\
\dot{S}_{\mathrm{ch}}= & D\left(S_{\mathrm{ch}}^{\mathrm{in}}-S_{\mathrm{ch}}\right)-f_{0}\left(S_{\mathrm{ch}}, S_{\mathrm{H}_{2}}\right) X_{\mathrm{ch}} \\
\dot{S}_{\mathrm{ph}}= & D\left(S_{\mathrm{ph}}^{\mathrm{in}}-S_{\mathrm{ph}}\right)+\frac{224}{208}\left(1-Y_{\mathrm{ch}}\right) f_{0}\left(S_{\mathrm{ch}}, S_{\mathrm{H}_{2}}\right) X_{\mathrm{ch}}-f_{1}\left(S_{\mathrm{ph}}, S_{\mathrm{H}_{2}}\right) X_{\mathrm{ph}} \\
\dot{S}_{\mathrm{H}_{2}}= & D\left(S_{\mathrm{H}_{2}}^{\mathrm{in}}-S_{\mathrm{H}_{2}}\right)-\frac{16}{208} f_{0}\left(S_{\mathrm{ch}}, S_{\mathrm{H}_{2}}\right) X_{\mathrm{ch}}+\frac{32}{224}\left(1-Y_{\mathrm{ph}}\right) f_{1}\left(S_{\mathrm{ph}}, S_{\mathrm{H}_{2}}\right) X_{\mathrm{ph}} \\
& -f_{2}\left(S_{\mathrm{H}_{2}}\right) X_{\mathrm{H}_{2}},
\end{aligned}\right.
$$

where $Y_{\mathrm{ch}}, Y_{\mathrm{ph}}$ and $Y_{\mathrm{H}_{2}}$ are the yield coefficients, respectively; 224/208 (1- $\left.Y_{\mathrm{ch}}\right)$ represents the fraction of chlorophenol converted to phenol; 32/224 $\left(1-Y_{\mathrm{ph}}\right)$ represents the fraction of phenol that is transformed to hydrogen and 16/208 represents the fraction of hydrogen consumed by the chlorophenol degrader. The biological parameter 
values, used in [26], are provided in Table 15. Following [20], the rescaling of the variables (G.1) and (G.2) can reduce (4.2) to the form (1.1), that is, the yields coefficients in (4.2) are normalized to one, except one of them which is equal to $\omega \simeq 0.53$. Under this rescaling (G.1) and (G.2), the growth functions (4.1) take the form (G.3) keeping their form of a double Monod, a Monod with product inhibition, and a Monod kinetics, respectively, so that Hypotheses (H1) to (H8) are satisfied. Therefore, with $\omega<1$, Theorem 3.1 apply and give rigorous proofs for the results of [26], on existence and stability of steady states, which, for the most part, have only been obtained numerically. See Appendix $G$ for the details.

In the following, we consider $S_{\mathrm{ph}}^{\text {in }}=0$ and $S_{\mathrm{H}_{2}}^{\text {in }}=2.67 \times 10^{-5}$, corresponding to Fig. 3(a) in [26] and we fix $S_{\mathrm{ch}}^{\mathrm{in}}=0.1$. Then, we determine the bifurcation diagram, where the operating parameter $D$ is the bifurcation parameter. Our aim is to compare our results to those of [26] and to see if there are interesting phenomena that were not detected in the operating diagram depicted in Fig. 3(a) of [26], see Remark 4.2. Using Theorem 3.1, we have the following result, which is supported by numerical experimentation and is proved in Appendix D.

Proposition 4.1. Let $S_{\mathrm{ph}}^{\mathrm{in}}=0, S_{\mathrm{H}_{2}}^{\text {in }}=2.67 \times 10^{-5}$ and $S_{\mathrm{ch}}^{\text {in }}=0.1$. In this case, SS7 and SS8 do not exist. Using the biological parameter values in Table 15, the bifurcation values $\delta_{i}, i=1, \ldots, 7$ are provided in Table 4 . The bifurcation analysis of (4.2) according to $D$ is given in Table 5 . The bifurcation types at the critical values $\delta_{i}$ are defined in Table 6 .

TABLE 4

Critical parameter values $\delta_{i}$, for $i=1, \ldots, 7$ where $Y$ is defined in Appendix $G, r_{5}$ in Table 2 while all other functions are given in Table 8.

\begin{tabular}{ll} 
Definition & Value \\
\hline$\delta_{1}$ is the largest root of equation $r_{5}=0$ & 0.010412 \\
$\delta_{2}$ is the root of $\phi_{2}(D)-S_{\mathrm{H}_{2}}^{\text {in }}-(1-\omega) Y S_{\mathrm{ch}}^{\text {in }}=0$ & 0.068641 \\
$\delta_{3}$ is the root of $\phi_{1}(D)-S_{\mathrm{H}_{2}}^{\text {in }}-(1-\omega) Y S_{\mathrm{ch}}^{\text {in }}=0$ & 0.068814 \\
$\delta_{4}$ is the root of $S_{\mathrm{H}_{2}}^{\text {in }}+\omega\left(\varphi_{0}(D)-Y S_{\mathrm{ch}}^{\text {in }}\right)-M_{2}\left(D+a_{2}\right)=0$ & 0.267251 \\
$\delta_{5}$ is the root of $\varphi_{0}(D)-Y S_{\mathrm{ch}}^{\text {in }}=0$ & 0.267636 \\
$\delta_{6}=\mu_{0}\left(Y S_{\mathrm{ch}}^{\text {in }}, S_{\mathrm{H}_{2}}^{\mathrm{in}}\right)-a_{0}$ & 0.327130 \\
$\delta_{7}=\mu_{2}\left(S_{\mathrm{H}_{2}}^{\mathrm{in}}\right)-a_{2}$ & 1.064526 \\
\hline
\end{tabular}

TABLE 5

Existence and stability of steady states, with respect to $D$. The bifurcation values $\delta_{i}, i=1, \ldots, 7$ are given in Table 4. The letter $S$ (resp. U) means that the corresponding steady state is stable (resp. unstable). No letter means that the steady state does not exist.

\begin{tabular}{l|lllllll} 
Interval & SS1 & SS2 & SS3 & SS4 $^{1}$ & SS4 $^{2}$ & SS5 & SS6 \\
\hline $0<D<\delta_{1}$ & $\mathrm{U}$ & $\mathrm{U}$ & $\mathrm{S}$ & $\mathrm{U}$ & $\mathrm{U}$ & & $\mathrm{U}$ \\
$\delta_{1}<D<\delta_{2}$ & $\mathrm{U}$ & $\mathrm{U}$ & $\mathrm{S}$ & $\mathrm{U}$ & $\mathrm{U}$ & & $\mathrm{S}$ \\
$\delta_{2}<D<\delta_{3}$ & $\mathrm{U}$ & $\mathrm{U}$ & $\mathrm{S}$ & $\mathrm{U}$ & $\mathrm{S}$ & & \\
$\delta_{3}<D<\delta_{4}$ & $\mathrm{U}$ & $\mathrm{U}$ & $\mathrm{S}$ & & & & \\
$\delta_{4}<D<\delta_{5}$ & $\mathrm{U}$ & $\mathrm{U}$ & $\mathrm{U}$ & & & $\mathrm{S}$ & \\
$\delta_{5}<D<\delta_{6}$ & $\mathrm{U}$ & $\mathrm{S}$ & $\mathrm{U}$ & & & & \\
$\delta_{6}<D<\delta_{7}$ & $\mathrm{U}$ & $\mathrm{S}$ & & & & & \\
$\delta_{7}<D$ & $\mathrm{~S}$ & & & & & &
\end{tabular}


TABLE 6

Bifurcation types corresponding to the critical values of $\delta_{i}, i=1, \ldots, 7$, defined in Table 4 . There exists also a critical value $\delta^{*} \simeq 0.009879<\delta_{1}$ corresponding to the value of $D$ where the stable limit cycle disappears when $D$ is increasing.

Bifurcation types

\begin{tabular}{ll}
\hline$\delta^{*}$ & Disappearance of the stable limit cycle \\
$\delta_{1}$ & Supercritical Hopf bifurcation \\
$\delta_{2}$ & Transcritical bifurcation of $\mathrm{SS} 4^{2}$ and $\mathrm{SS} 6$ \\
$\delta_{3}$ & Saddle-node bifurcation of $\mathrm{SS} 4^{1}$ and $\mathrm{SS} 4^{2}$ \\
$\delta_{4}$ & Transcritical bifurcation of $\mathrm{SS} 3$ and SS5 \\
$\delta_{5}$ & Transcritical bifurcation of SS2 and SS5 \\
$\delta_{6}$ & Transcritical bifurcation of $\mathrm{SS} 1$ and SS3 \\
$\delta_{7}$ & Transcritical bifurcation of $\mathrm{SS} 1$ and SS2
\end{tabular}

Figure 4.1 shows the one-parameter bifurcation diagram of $X_{\mathrm{ch}}$ versus $D$ in system (4.2). The magnifications of the bifurcation diagram are illustrated in Figure 4.1(bc-d) showing the disappearance of the limit cycle at $\delta^{*}$, the Hopf bifurcation at $\delta_{1}$, the transcritical bifurcations at $\delta_{2}, \delta_{4}$ and $\delta_{5}$ and the saddle-node bifurcation at $\delta_{3}$. In Figure 4.1, SS1 and SS2 cannot be distinguished since they have both a zero $X_{\mathrm{ch}^{-}}$ component. As SS2 is stable and SS1 is unstable for $D<\delta_{7}$, the $X_{\mathrm{ch}}=0$ axis is plotted in blue as the color of SS2 in Table 7.

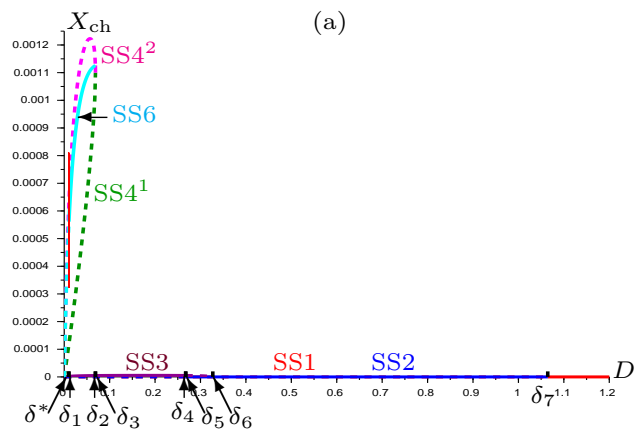

(c)

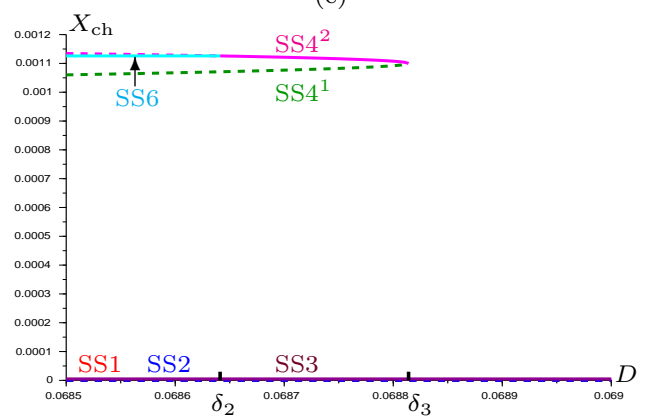

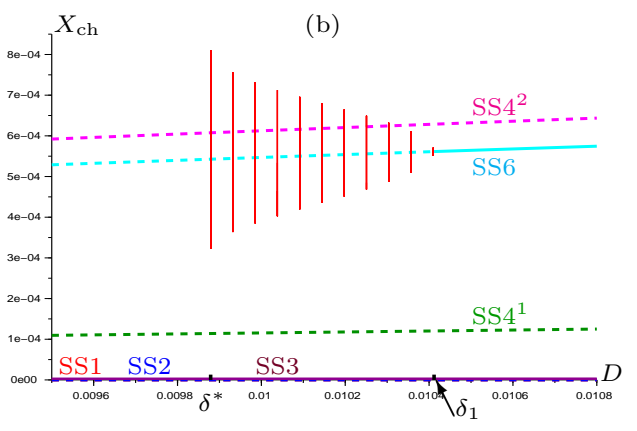

(d)

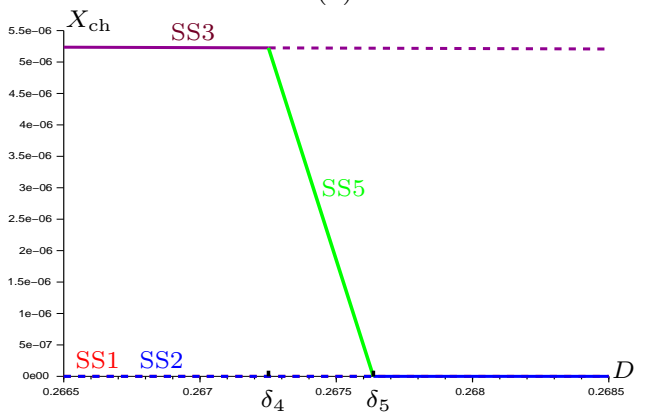

FIG. 4.1. (a) Bifurcation diagram of $X_{\mathrm{ch}}$ versus $D \in[0,1.2]$ in model (4.2). (b) Magnification on the appearance and disappearance of stable limit cycles for $D \in[0.0095,0.0108]$. (c) Magnification on the transcritical bifurcation at $D=\delta_{2}$ and the saddle-node bifurcation at $D=\delta_{3}$ for $D \in$ $[0.0685,0.069]$. (d) Magnification on the transcritical bifurcations for $D \in[0.2665,0.2685]$.

Remark 4.2. Not all of the behaviors described in Table 5 were reported in [26]. 
TABLE 7

Colors used in Figures 4.1 and E.1. The solid (resp. dashed) lines are used for stable (resp. unstable) steady states.

\begin{tabular}{lllllll} 
SS1 & SS2 & SS3 & SS4 $^{1}$ & SS4 $^{2}$ & SS5 & SS6 \\
\hline Red & Blue & Purple & Dark Green & Magenta & Green & Cyan
\end{tabular}

For $S_{\mathrm{ch}}^{\text {in }}=0.1$, the operating diagram of Fig. 3(a) in [26] predicts only three possible behaviors: the stability of SS2, the stability of SS3 and the bistability between SS3 and SS6. Note that the destabilization of SS6 via a Hopf bifurcation with emergence of a stable limit cycle has not been observed in [26]. Moreover, the region of existence and stability of SS5, which was depicted in Fig. 3(b) of [26] in the case where $S_{\mathrm{H}_{2}}^{\text {in }}=$ $2.67 \times 10^{-2}$, was not reported in Fig. 3(a) of [26]. Our results show that this region also exists when $S_{\mathrm{H}_{2}}^{\text {in }}=2.67 \times 10^{-5}$, and explain why it was not detected by the numerical analysis given in Fig. 3(a) of [26]: SS5 occurs in a very small region since, for $S_{\mathrm{ch}}^{\text {in }}=0.1$ it corresponds to $\delta_{4}<D<\delta_{5}$, where $\delta_{4} \simeq 0.267251$ and $\delta_{5} \simeq 0.267636$, with $\delta_{5}-\delta_{4}$ of order $10^{-4}$. However, while from a mathematical point of view the diagram shown in [26] is incorrectly labeled, in biological terms, such a small region of SS5 would likely not be attained.

To compare our results to those achieved in [16], we determine the bifurcation diagram in Appendix E according to the bifurcation parameter $S_{\mathrm{ch}}^{\mathrm{in}}$. Further, numerical simulations are presented in Figures 4.2 to 4.4 (see also Figures H.1 to H.4) to illustrate our findings, where the bifurcation values $\sigma_{5}, \sigma_{6}$ and $\sigma^{*}$ of $S_{\mathrm{ch}}^{\text {in }}$ are provided in Tables 10 and 12, respectively. We illustrate, in particular, the interesting three cases where the steady states $\mathrm{SS} 1, \mathrm{SS} 2, \mathrm{SS} 4^{1}$ and $\mathrm{SS} 4^{2}$ are unstable:

- For $S_{\mathrm{ch}}^{\mathrm{in}} \in\left(\sigma_{5}, \sigma^{*}\right)$, the numerical simulations done for various positive initial conditions permit to conjecture the global asymptotic stability of SS3 (see Figure 4.2).

- For $S_{\mathrm{ch}}^{\mathrm{in}} \in\left(\sigma^{*}, \sigma_{6}\right)$, the system exhibits a bistability with two basins of attraction: one toward the stable limit cycle and the second toward SS3. Figure 4.3 illustrates that the trajectories in pink and blue converge toward the stable limit cycle in red, while the green trajectory converges toward SS3. For the initial condition in Table 14, the time course in Figure H.1 illustrates the positive, periodic solution representing the coexistence of the three species. The sustained oscillations prove the stability of the limit cycle. However, Figure H.2 shows the time course of the green trajectory in Figure 4.3.

- For $S_{\mathrm{ch}}^{\text {in }}>\sigma_{6}$, the system exhibits a bistability between SS6 and SS3. Figure 4.4 shows that the blue trajectory converges to the stable focus SS6, while the green trajectory converges to SS3. Figures H.3 and H.4 illustrate the time courses corresponding to the blue and the green trajectories in Figure 4.4, respectively. Numerical simulations have shown that the stable limit cycle disappears at the critical value $\sigma^{*} \in\left(\sigma_{5}, \sigma_{6}\right)$ as $S_{\mathrm{ch}}^{\text {in }}$ decreases. Similarly to the numerical study of the bifurcation diagram with respect to the parameter $D$ in [23] in the case without maintenance and $s_{1}^{\text {in }}=s_{2}^{\text {in }}=0$, we conjecture that in our case also the stable limit cycle disappears through a saddle-node bifurcation with another unstable limit cycle when $S_{\mathrm{ch}}^{\mathrm{in}}$ decreases.

5. Conclusion. In this study, we discussed the dynamics of three interacting microbial species describing a chlorophenol-mineralising three-tiered 'food web' in the chemostat (4.2), introduced by Wade et al. [26] following previous work on a 


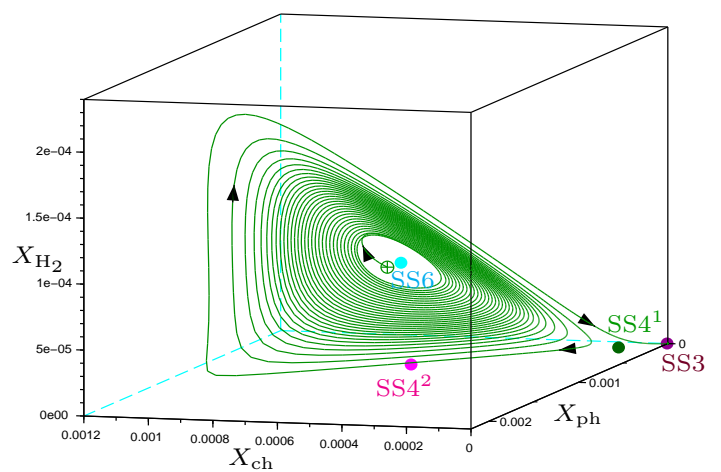

FIG. 4.2. Case $S_{\mathrm{ch}}^{\mathrm{in}}=0.098<\sigma^{*}$ : the solution of (4.2) converges to SS3.

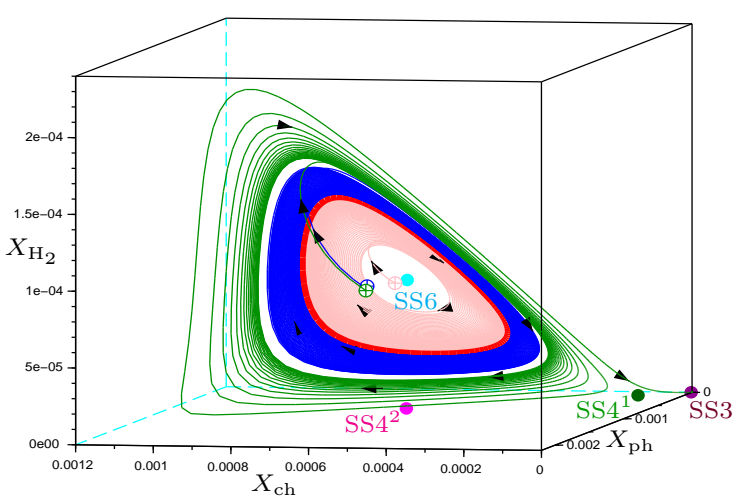

FIG. 4.3. Case $\sigma^{*}<S_{\mathrm{ch}}^{\mathrm{in}}=0.0995<\sigma_{6}:$ bistability with convergence either to the stable limit cycle (in red) or to SS3.

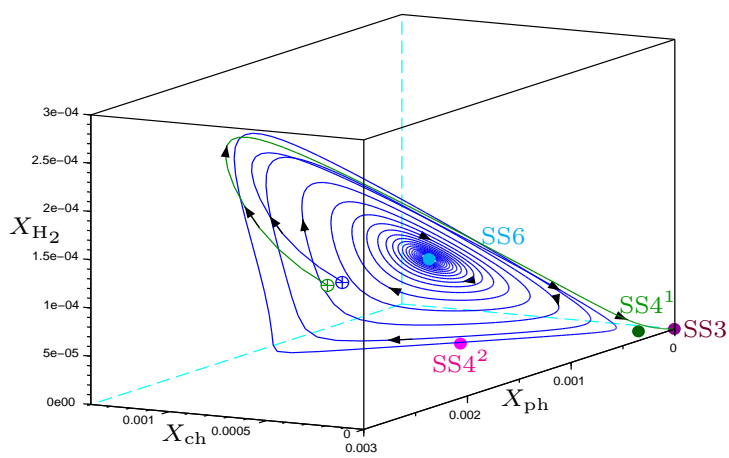

FIG. 4.4. Case $\sigma_{6}<S_{\mathrm{ch}}^{\mathrm{in}}=0.11$ : bistability with convergence either to SS6 or to SS3.

two-tiered model [29]. The existence and stability of the steady states of model (4.2) have been analyzed as a function of the operating parameters (input substrate concentrations and dilution rate), using numerical tools and specific values of the biological parameters.

In this paper, we gave a complete analysis of the dynamics of the model (1.1) which generalizes (4.2) by allowing a larger class of growth functions. The existence of the steady states was analytically characterized in [16] where it was shown that model (1.1) can have up to eight types of steady states: the washout steady state 
denoted by SS1, six types of boundary steady states where one or two degrader populations are extinct denoted by SS2, SS3, SS4, SS5, SS7 and SS8, and a positive steady state, denoted by SS6, where all microbial populations coexist. When they exist, all steady states are unique, except the steady state SS4 where chlorophenol and phenol degraders are maintained and the hydrogen degrader is eliminated.

Here, we focus on the stability of steady states. We have managed to characterize the stability in this six-dimensional system, although it is generally accepted that the Routh-Hurwitz theorem is intractable beyond five dimensions. For this, we have used the Liénard-Chipart stability criterion to simplify the mathematical analysis by reducing considerably the number of the Routh-Hurwitz conditions to check. For SS1, SS2, SS3 and SS7, the stability conditions are determined explicitly. For SS4, SS5 and SS8, we explicitly characterize the stability conditions using the Liénard-Chipart stability criterion. For SS6, the stability is given with respect to the signs of the Liénard-Chipart coefficients by using numerical experimentation (see Appendix D) to plot these coefficients, whose signs cannot be determined analytically. As shown in Appendix G, our presentation of the existence and stability issue fully clarifies the numerical study made in [26] on the three-tiered 'food web' model (4.2).

Our work extends all results on the stability of the existing literature [8, 16, 20,23], which were obtained only in the case without maintenance, where the six-dimensional system (1.1) can be reduced to a three-dimensional one. We show that for SS4, which can exist in two forms, at most one steady state can be stable, a fact that was already noticed (when maintenance is not included in the model) in the particular case without phenol and hydrogen input concentrations, studied in [20] and in the general case, where these input concentrations are added, studied in [16].

We highlighted several possible asymptotic behaviors in this six-dimensional system, including the bistability between the positive steady state and a boundary steady state, or the bistability between a positive limit cycle and a boundary steady state, so that the long term behavior depends on the initial condition. We proved that the positive steady state of coexistence of all species can be unstable and we give numerical evidence for the supercritical Hopf bifurcation, in the case including chlorophenol and hydrogen input concentrations. The possibility of the Hopf bifurcation of the positive steady state was previously observed in [20] in the case without phenol and hydrogen input concentrations.

In order to gain more insight into the behavior of the system, we give a bifurcation diagram with the dilution rate as the bifurcating parameter (see Figure 4.1) showing that one of the operating diagrams obtained numerically in [26] has omitted important transition phenomena between steady states. If the dilution rate is too low, only the chlorophenol degrader is maintained (SS3 is the only stable steady state). Increasing slightly the dilution rate $D$, the system exhibits a bistability behavior where either only the chlorophenol degrader is maintained (SS3 is stable) or the coexistence of three microbial species may occur around periodic oscillations (SS6 is unstable and a stable limit cycle exists). Increasing a little more $D$, the system exhibits a bistability behavior where either only the chlorophenol degrader is maintained or the coexistence of three microbial species occurs at the positive steady state (SS3 and SS6 are both stable). Increasing further $D$, the system exhibits a bistability between only the chlorophenol degrader and both the chlorophenol and phenol degraders (SS3 and SS4 ${ }^{2}$ are both stable). Rising a little more the value of $D$, only the chlorophenol degrader is maintained. Then, only the chlorophenol and hydrogen degraders are maintained (SS5 is the only stable steady state). Adding a little more, both the chlorophenol and phenol degraders are eliminated from the reactor and only the hydrogen degrader is 
maintained, since $S_{\mathrm{H}_{2}}^{\mathrm{in}}>0$ (SS2 is the only stable steady state). For higher dilution rate, there is washout of all three microbial populations (SS1 is the only stable steady state).

Our results show that with the exception of SS6, the maintenance does not destabilize the steady states. To make our theoretical results useful in practice, it would be necessary to have the description of the operating diagrams that give the regions of existence and stability of the steady states, in the space of the operating parameters. In a future work, we will use our results to determine analytically the operating diagrams in the cases with and without maintenance. These operating diagrams will also allow us to answer the delicate question of whether or not SS6 can be destabilized by including maintenance terms. Even without maintenance, this steady state can be stable or unstable depending on the values of the operating parameters. Does the introduction of maintenance modify the boundary between the region of stability and the region of instability, or does it make more complex phenomena appear?

Appendix A. Auxiliary functions. For the description of the steady states given in Table 1, together with the statement of their conditions of existence and stability, we need to define some auxiliary functions that are listed in Table 8. Using Hypotheses (H1) to (H7), the existence and definition domains of these functions are all relatively straightforward and can be found as in [20].

TABLE 8

Notations, intervals and auxiliary functions.

Definition

\begin{tabular}{|c|c|}
\hline $\begin{array}{l}s_{i}=M_{i}\left(y, s_{2}\right) \\
i=0,1\end{array}$ & $\begin{array}{l}\text { Let } s_{2} \geq 0 . s_{i}=M_{i}\left(y, s_{2}\right) \text { is the unique solution of } \\
\mu_{i}\left(s_{i}, s_{2}\right)=y, \text { for all } 0 \leq y<\mu_{i}\left(+\infty, s_{2}\right)\end{array}$ \\
\hline$s_{2}=M_{2}(y)$ & $\begin{array}{l}s_{2}=M_{2}(y) \text { is the unique solution of } \\
\mu_{2}\left(s_{2}\right)=y, \text { for all } 0 \leq y<\mu_{2}(+\infty)\end{array}$ \\
\hline$s_{2}=M_{3}\left(s_{0}, z\right)$ & $\begin{array}{l}\text { Let } s_{0} \geq 0 . s_{2}=M_{3}\left(s_{0}, z\right) \text { is the unique solution of } \\
\mu_{0}\left(s_{0}, s_{2}\right)=z, \text { for all } 0 \leq z<\mu_{0}\left(s_{0},+\infty\right)\end{array}$ \\
\hline $\begin{array}{l}s_{2}^{i}=s_{2}^{i}(D) \\
i=0,1\end{array}$ & $\begin{array}{l}s_{2}^{i}=s_{2}^{i}(D) \text { is the unique solution of } \mu_{i}\left(+\infty, s_{2}\right)=D+a_{i} \text {, for all } \\
D+a_{0}<\mu_{0}(+\infty,+\infty), \mu_{1}(+\infty,+\infty)<D+a_{1}<\mu_{1}(+\infty, 0) \text {, resp. }\end{array}$ \\
\hline$I_{1}, \quad I_{2}$ & $I_{1}=\left\{D \geq 0: s_{2}^{0}<s_{2}^{1}\right\}, I_{2}=\left\{D \in I_{1}: s_{2}^{0}<M_{2}\left(D+a_{2}\right)<s_{2}^{1}\right\}$ \\
\hline$\Psi\left(s_{2}, D\right)$ & $\begin{array}{l}\Psi\left(s_{2}, D\right)=(1-\omega) M_{0}\left(D+a_{0}, s_{2}\right)+M_{1}\left(D+a_{1}, s_{2}\right)+s_{2}, \\
\text { for all } D \in I_{1} \text { and } s_{2}^{0}<s_{2}<s_{2}^{1}\end{array}$ \\
\hline$\phi_{1}(D)$ & $\phi_{1}(D)=\inf _{s_{2}^{0}<s_{2}<s_{2}^{1}} \Psi\left(s_{2}, D\right), \quad$ for all $D \in I_{1}$ \\
\hline$\phi_{2}(D)$ & $\phi_{2}(D)=\Psi\left(M_{2}\left(D+a_{2}\right), D\right), \quad$ for all $D \in I_{2}$ \\
\hline$\phi_{3}(D)$ & $\phi_{3}(D)=\frac{\partial \Psi}{\partial s_{2}}\left(M_{2}\left(D+a_{2}\right), D\right), \quad$ for all $D \in I_{2}$ \\
\hline$J_{0}, \quad J_{1}$ & $J_{0}=\left(\max \left(0, s_{0}^{\text {in }}-s_{2}^{\text {in }} / \omega\right), s_{0}^{\text {in }}\right), \quad J_{1}=\left(0, s_{1}^{\text {in }}\right)$ \\
\hline$\psi_{0}\left(s_{0}\right)$ & $\psi_{0}\left(s_{0}\right)=\mu_{0}\left(s_{0}, s_{2}^{\text {in }}-\omega\left(s_{0}^{\text {in }}-s_{0}\right)\right)$, for all $s_{0} \geq \max \left(0, s_{0}^{\text {in }}-s_{2}^{\text {in }} / \omega\right)$ \\
\hline$\psi_{1}\left(s_{1}\right)$ & $\psi_{1}\left(s_{1}\right)=\mu_{1}\left(s_{1}, s_{2}^{\text {in }}+s_{1}^{\text {in }}-s_{1}\right), \quad$ for all $s_{1} \in\left[0, s_{1}^{\text {in }}+s_{2}^{\text {in }}\right]$ \\
\hline $\begin{array}{l}\varphi_{i}(D) \\
i=0,1\end{array}$ & $\begin{array}{l}\varphi_{i}(D)=M_{i}\left(D+a_{i}, M_{2}\left(D+a_{2}\right)\right), \text { resp., for all, } \\
D \in\left\{D \geq 0: s_{2}^{0}<M_{2}\left(D+a_{2}\right)\right\}, D \in\left\{D \geq 0: M_{2}\left(D+a_{2}\right)<s_{2}^{1}\right\}\end{array}$ \\
\hline
\end{tabular}

Appendix B. Liénard-Chipart stability criterion. Note that conditions in the stability criterion of Liénard and Chipart (see Gantmacher [10], Theorem 11) represent almost half that of the Routh-Hurwitz theorem which facilitates the study of asymptotic behavior of dynamic systems especially for dimensions beyond five. It is known that for a polynomial of degree four the Routh-Hurwitz conditions can be written as in the following Lemma, see, for instance, Theorem 11 [6]. 
Lemma B.1. Consider the fourth-order polynomial $P(\lambda)$ with real coefficients given by:

$$
P(\lambda)=c_{0} \lambda^{4}+c_{1} \lambda^{3}+c_{2} \lambda^{2}+c_{3} \lambda+c_{4}
$$

All of the roots of the polynomial $P(\lambda)$ have negative real part if and only if

$$
c_{i}>0, \quad \text { for } \quad i=1,3,4, \quad \text { and } \quad r_{1}=c_{3}\left(c_{1} c_{2}-c_{0} c_{3}\right)-c_{1}^{2} c_{4}>0 .
$$

The following Lemma gives the conditions of stability for a six-dimensional dynamic system.

Lemma B.2. Consider the six-order polynomial $P(\lambda)$ with real coefficients given by:

$$
P(\lambda)=c_{0} \lambda^{6}+c_{1} \lambda^{5}+c_{2} \lambda^{4}+c_{3} \lambda^{3}+c_{4} \lambda^{2}+c_{5} \lambda+c_{6} .
$$

All of the roots of the polynomial $P(\lambda)$ have negative real part if and only if

$$
c_{i}>0, \quad i=1,3,5,6, \quad r_{4}>0 \quad \text { and } \quad r_{5}>0,
$$

where $r_{4}=r_{1} r_{2}-r_{0} r_{3}$ and $r_{5}=r_{3} r_{4}-c_{1} c_{6} r_{2}^{2}$, with

$$
r_{0}=c_{1} c_{2}-c_{0} c_{3}, r_{1}=c_{1} c_{4}-c_{0} c_{5}, r_{2}=c_{3} r_{0}-c_{1} r_{1} \text { and } r_{3}=c_{5} r_{0}-c_{1}^{2} c_{6} .
$$

Proof. From the Liénard-Chipart stability criterion, all of the roots of the polynomial $P$ have negative real part if and only if

$$
c_{i}>0, \quad i=1,3,5,6, \quad \operatorname{det}\left(\Delta_{2}\right)>0, \quad \operatorname{det}\left(\Delta_{4}\right)>0 \quad \text { and } \quad \operatorname{det}\left(\Delta_{6}\right)>0,
$$

where $\Delta_{2}, \Delta_{4}$ and $\Delta_{6}$ are the Hurwitz matrices defined by:

$\Delta_{2}=\left[\begin{array}{ll}c_{1} & c_{3} \\ c_{0} & c_{2}\end{array}\right], \Delta_{4}=\left[\begin{array}{cccc}c_{1} & c_{3} & c_{5} & 0 \\ c_{0} & c_{2} & c_{4} & c_{6} \\ 0 & c_{1} & c_{3} & c_{5} \\ 0 & c_{0} & c_{2} & c_{4}\end{array}\right], \Delta_{6}=\left[\begin{array}{cccccc}c_{1} & c_{3} & c_{5} & 0 & 0 & 0 \\ c_{0} & c_{2} & c_{4} & c_{6} & 0 & 0 \\ 0 & c_{1} & c_{3} & c_{5} & 0 & 0 \\ 0 & c_{0} & c_{2} & c_{4} & c_{6} & 0 \\ 0 & 0 & c_{1} & c_{3} & c_{5} & 0 \\ 0 & 0 & c_{0} & c_{2} & c_{4} & c_{6}\end{array}\right]$.

Conditions (B.3) are equivalent to

(B.4) $\quad c_{i}>0, i=1,3,5,6, r_{0}>0, r_{4}=r_{1} r_{2}-r_{0} r_{3}>0, r_{5}=r_{3} r_{4}-c_{1} c_{6} r_{2}^{2}>0$.

When all conditions (B.4) hold, the condition $r_{5}>0$ implies that $r_{3}>0$, that is, $c_{5} r_{0}>c_{6} c_{1}^{2}$ which implies that $r_{0}>0$. Hence, conditions (B.4) are equivalent to (B.2).

\section{Appendix C. Proofs.}

C.1. Proof of Theorem 3.1. The existence of the steady states is proven in [16]. The local stability of the steady states is determined by the eigenvalues of the Jacobian matrix of system (1.1) evaluated at the steady state. The Jacobian matrix of (1.1) corresponds to the $6 \times 6$ matrix:

$382 \quad \mathcal{J}=\left[\begin{array}{cccccc}J-D-a_{0} & 0 & 0 & E x_{0} & 0 & F x_{0} \\ 0 & K-D-a_{1} & 0 & 0 & G x_{1} & -H x_{1} \\ 0 & 0 & L-D-a_{2} & 0 & 0 & I x_{2} \\ -J & 0 & 0 & -D-E x_{0} & 0 & -F x_{0} \\ J & -K & 0 & E x_{0} & -D-G x_{1} & F x_{0}+H x_{1} \\ -\omega J & K & -L & -\omega E x_{0} & G x_{1} & -D-\omega F x_{0}-H x_{1}-I x_{2}\end{array}\right]$, 
where the functions $E, F, G, H, I, J, K$ and $L$ are defined by (3.1), and are evaluated at the steady state. The stability of the steady state is investigated by analyzing the real parts of the eigenvalues of $\mathcal{J}$, which are the roots of the characteristic polynomial.

For SS1, the characteristic polynomial is

$$
P_{1}(\lambda)=\left(\lambda-\lambda_{1}\right)\left(\lambda-\lambda_{2}\right)\left(\lambda-\lambda_{3}\right)(\lambda+D)^{3},
$$

where $\lambda_{1}=\mu_{0}\left(s_{0}^{\text {in }}, s_{2}^{\text {in }}\right)-D-a_{0}, \lambda_{2}=\mu_{1}\left(s_{1}^{\text {in }}, s_{2}^{\text {in }}\right)-D-a_{1}$ and $\lambda_{3}=\mu_{2}\left(s_{2}^{\text {in }}\right)-D-a_{2}$. Therefore, SS1 is stable if and only if $\lambda_{1}<0, \lambda_{2}<0$ and $\lambda_{3}<0$, that is, the stability conditions of SS1 in Table 3 hold.

For SS2, the characteristic polynomial is

$$
P_{2}(\lambda)=\left(\lambda-\lambda_{1}\right)\left(\lambda-\lambda_{2}\right)(\lambda+D)^{2}\left(\lambda^{2}+c_{1} \lambda+c_{2}\right),
$$

where $c_{1}=D+I x_{2}, c_{2}=L I x_{2}$ and

$$
\text { (C.1) } \lambda_{1}=\mu_{0}\left(s_{0}^{\text {in }}, M_{2}\left(D+a_{2}\right)\right)-D-a_{0}, \quad \lambda_{2}=\mu_{1}\left(s_{1}^{\text {in }}, M_{2}\left(D+a_{2}\right)\right)-D-a_{1} \text {, }
$$

Since $c_{1}>0$ and $c_{2}>0$, the real parts of the roots of the quadratic factor are negative. Therefore, SS2 is stable if and only if $\lambda_{1}<0$ and $\lambda_{2}<0$. Since $M_{0}$ and $M_{1}$ are increasing, these conditions are equivalent to the stability conditions of SS2 in Table 3.

For SS3, the characteristic polynomial is

$$
P_{3}(\lambda)=\left(\lambda-\lambda_{1}\right)\left(\lambda-\lambda_{2}\right)(\lambda+D)^{2}\left(\lambda^{2}+c_{1} \lambda+c_{2}\right),
$$

where

$\lambda_{1}=\mu_{1}\left(s_{0}^{\text {in }}-s_{0}+s_{1}^{\text {in }}, s_{2}^{\text {in }}-\omega\left(s_{0}^{\text {in }}-s_{0}\right)\right)-D-a_{1}, \lambda_{2}=\mu_{2}\left(s_{2}^{\text {in }}-\omega\left(s_{0}^{\text {in }}-s_{0}\right)\right)-D-a_{2}$, $c_{1}=D+(E+\omega F) x_{0}$ and $c_{2}=J(E+\omega F) x_{0}$, where $s_{0}$ is the solution in the interval $J_{0}$ of equation $\psi_{0}\left(s_{0}\right)=D+a_{0}$. Since $c_{1}>0$ and $c_{2}>0$, the real parts of the roots of the quadratic factor are negative. Therefore, SS3 is stable if and only if $\lambda_{1}<0$ and $\lambda_{2}<0$. The condition $\lambda_{1}<0$ is the first stability condition of SS3 in Table 3. Since $M_{2}$ is increasing, the condition $\lambda_{2}<0$ is equivalent to

(C.2) $s_{2}^{\text {in }}-\omega\left(s_{0}^{\text {in }}-s_{0}\right)<M_{2}\left(D+a_{2}\right) \Longleftrightarrow s_{0}<\left(M_{2}\left(D+a_{2}\right)-s_{2}^{\text {in }}\right) / \omega+s_{0}^{\text {in }}$.

As the function $\psi_{0}$ is increasing, (C.2) is equivalent to

$$
\psi_{0}\left(s_{0}\right)<\psi_{0}\left(\left(M_{2}\left(D+a_{2}\right)-s_{2}^{\text {in }}\right) / \omega+s_{0}^{\text {in }}\right) .
$$

From the definition of the function $\psi_{0}$ together with the condition $\psi_{0}\left(s_{0}\right)=D+a_{0}$ defining $s_{0}$, we deduce that (C.3) is equivalent to

$$
D+a_{0}<\mu_{0}\left(\left(M_{2}\left(D+a_{2}\right)-s_{2}^{\text {in }}\right) / \omega+s_{0}^{\text {in }}, M_{2}\left(D+a_{2}\right)\right) .
$$

Since $M_{0}$ is increasing, this condition is equivalent to the second stability condition of SS3 in Table 3.

For SS4, the characteristic polynomial is

$$
P_{4}(\lambda)=\left(\lambda-\lambda_{1}\right)(\lambda+D)\left(\lambda^{4}+c_{1} \lambda^{3}+c_{2} \lambda^{2}+c_{3} \lambda+c_{4}\right),
$$

where $\lambda_{1}=\mu_{2}\left(s_{2}\right)-D-a_{2}$ with $s_{2}$ is defined in Table 1 and the coefficients $c_{i}$ for $i=1, \ldots, 4$ are given by

$$
\begin{aligned}
& c_{1}=2 D+(E+\omega F) x_{0}+(G+H) x_{1}, \\
& c_{2}=D^{2}+(E+\omega F)(D+J) x_{0}+(G+H)(D+K) x_{1}+(E(G+H)-(1-\omega) F G) x_{0} x_{1}, \\
& c_{3}=D(E+\omega F) J x_{0}+D(G+H) K x_{1}+(E(G+H)-(1-\omega) F G)(J+K) x_{0} x_{1}, \\
& c_{4}=(E(G+H)-(1-\omega) F G) J K x_{0} x_{1} .
\end{aligned}
$$


From Lemma B.1, all of the roots of the fourth order polynomial have negative real parts if and only if

$$
c_{i}>0, \quad \text { for } \quad i=1,3,4 \text { and } \quad r_{1}=c_{1} c_{2} c_{3}-c_{1}^{2} c_{4}-c_{3}^{2}>0 .
$$

We always have $c_{1}>0$. Moreover, $c_{3}>0$ and $c_{4}>0$ if and only if

$$
E(G+H)-(1-\omega) F G>0 .
$$

Let us denote

$$
A=G+H, \quad B=\frac{E(G+H)-(1-\omega) F G}{G+H} \quad \text { and } \quad C=\frac{G+\omega H}{G+H} F .
$$

Note that $B>0$ if and only if condition (C.5) is satisfied. Then, we can write $c_{i}$, for $i=1, \ldots, 4$ as follows:

$$
\begin{aligned}
& c_{1}=2 D+(B+C) x_{0}+A x_{1}, \\
& c_{2}=D^{2}+(B+C)(D+J) x_{0}+A(D+K) x_{1}+A B x_{0} x_{1}, \\
& c_{3}=D(B+C) J x_{0}+D A K x_{1}+A B(J+K) x_{0} x_{1}, \quad c_{4}=A B J K x_{0} x_{1} .
\end{aligned}
$$

We can write $r_{1}$ as follows:

$$
\begin{aligned}
r_{1}= & D J\left[(D+J)(B+C)^{3}-B^{3} J\right] x_{0}^{3}+D^{2} A^{3} K x_{1}^{3}+B^{2} A^{2}(B+C)(J+K) x_{0}^{3} x_{1}^{2}+B^{2} A^{3}(J+K) x_{0}^{2} x_{1}^{3} \\
& +B A\left[D(2 J+K)(B+C)^{2}+C J^{2}(2 B+C)\right] x_{0}^{3} x_{1}+D B A^{3}(J+2 K) x_{0} x_{1}^{3}+3 D^{3} A^{2} K x_{1}^{2} \\
& +D^{2} J\left[3 D(B+C)^{2}+C J(2 B+C)\right] x_{0}^{2}+B A^{2}\left[D(J+K)(5 B+3 C)+C\left(J^{2}+K^{2}\right)\right] x_{0}^{2} x_{1}^{2} \\
& +D A\left[C\left(D C(2 J+K)+C J(J+2 K)+D B(9 J+5 K)+2 B J^{2}\right)+D B^{2}(7 J+4 K)\right] x_{0}^{2} x_{1} \\
& +D A^{2}[D B(4 J+7 K)+C K(2 J+K)+D C(J+2 K)] x_{0} x_{1}^{2}+2 D^{4} J(B+C) x_{0}+2 D^{4} A K x_{1} \\
& +D^{2} A[D(J+K)(5 B+3 C)+2 C J K] x_{0} x_{1}+\left(D^{2}+D B x_{0}+D A x_{1}+B A x_{0} x_{1}\right)\left(B J x_{0}-A K x_{1}\right)^{2} .
\end{aligned}
$$

Hence, conditions (C.4) are verified if and only if (C.5) is satisfied. Let us prove that condition (C.5) is equivalent to $\frac{\partial \Psi}{\partial s_{2}}\left(s_{2}, D\right)>0$. Let $s_{2}>0$. Under (H4) and (H5), we have

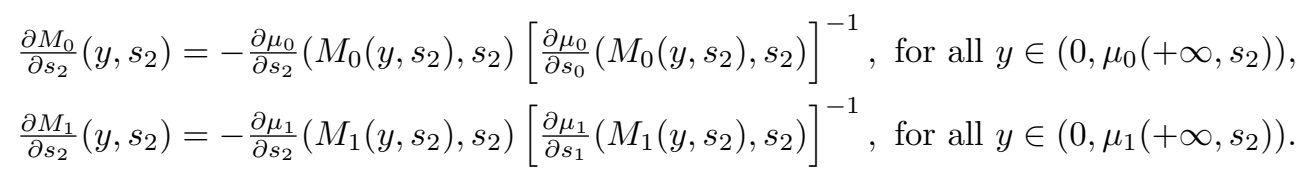

Using (3.1), we obtain

$$
\frac{\partial M_{0}}{\partial s_{2}}\left(D+a_{0}, s_{2}\right)=-\frac{F}{E} \quad \text { and } \quad \frac{\partial M_{1}}{\partial s_{2}}\left(D+a_{1}, s_{2}\right)=\frac{H}{G} .
$$

Moreover, we have for all $s_{2} \in\left(s_{2}^{0}, s_{2}^{1}\right)$ and $D \in I_{1}$,

$$
\frac{\partial \Psi}{\partial s_{2}}\left(s_{2}, D\right)=(1-\omega) \frac{\partial M_{0}}{\partial s_{2}}\left(D+a_{0}, s_{2}\right)+\frac{\partial M_{1}}{\partial s_{2}}\left(D+a_{1}, s_{2}\right)+1 .
$$

Using (C.6), it follows that

$$
\frac{\partial \Psi}{\partial s_{2}}\left(s_{2}, D\right)=-\frac{F}{E}(1-\omega)+\frac{H}{G}+1=\frac{E(G+H)-(1-\omega) F G}{E G} .
$$

Since $\mathrm{E}$ and $\mathrm{G}$ are positive, condition (C.5) is equivalent to $\frac{\partial \Psi}{\partial s_{2}}\left(s_{2}, D\right)>0$. Consequently, since $\mu_{2}$ is increasing, it follows that, SS4 is stable if and only if

$$
s_{2}<M_{2}\left(D+a_{2}\right) \text { and } \frac{\partial \Psi}{\partial s_{2}}\left(s_{2}, D\right)>0,
$$


which is equivalent to the stability condition in Table 3 because this first condition of (C.7) is equivalent the first and the second one of SS4 in Table 3 (similarly to the proof of Theorem 2 in [16]).

For SS5, the characteristic polynomial is

$$
P_{5}(\lambda)=\left(\lambda-\lambda_{1}\right)(\lambda+D)\left(\lambda^{4}+c_{1} \lambda^{3}+c_{2} \lambda^{2}+c_{3} \lambda+c_{4}\right),
$$

where $\lambda_{1}=\mu_{1}\left(s_{0}^{\text {in }}+s_{1}^{\text {in }}-M_{0}\left(D+a_{0}, M_{2}\left(D+a_{2}\right)\right), M_{2}\left(D+a_{2}\right)\right)-D-a_{1}$ and the coefficients $c_{i}$ are given by:

$$
\begin{aligned}
& c_{1}=2 D+(E+\omega F) x_{0}+I x_{2}, \\
& c_{2}=D^{2}+(E+\omega F)(D+J) x_{0}+I(D+L) x_{2}+E I x_{0} x_{2}, \\
& c_{3}=D(E+\omega F) J x_{0}+D I L x_{2}+E I(J+L) x_{0} x_{2} \text { and } c_{4}=E I J L x_{0} x_{2} .
\end{aligned}
$$

From Lemma B.1, the roots of the fourth order polynomial are of negative real parts if and only if

$$
c_{i}>0, \quad \text { for } i=1,3,4 \text { and } \quad r_{1}=c_{1} c_{2} c_{3}-c_{1}^{2} c_{4}-c_{3}^{2}>0 .
$$

We always have $c_{i}>0$ for $i=1,3,4$. We can write $r_{1}$ as follows:

$$
\begin{aligned}
r_{1}= & D J\left[(D+J)(E+\omega F)^{3}-E^{3} J\right] x_{0}^{3}+D^{2} I^{3} L x_{2}^{3}+E^{2} I^{2}(E+\omega F)(J+L) x_{0}^{3} x_{2}^{2}+D E I^{3}(J+2 L) x_{0} x_{2}^{3} \\
& +E^{2} I^{3}(J+L) x_{0}^{2} x_{2}^{3}+E I\left[D(2 J+L)(E+\omega F)^{2}+\omega F J^{2}(2 E+\omega F)\right] x_{0}^{3} x_{2}+3 D^{3} I^{2} L x_{2}^{2} \\
& +D^{2} J\left[3 D(E+\omega F)^{2}+F \omega J(2 E+\omega F)\right] x_{0}^{2}+E I^{2}\left[D(J+L)(5 E+3 \omega F)+F \omega\left(J^{2}+L^{2}\right)\right] x_{0}^{2} x_{2}^{2} \\
& +D I\left[F \omega\left(D F \omega(2 J+L)+F \omega J(J+2 L)+D E(9 J+5 L)+2 E J^{2}\right)+D E^{2}(7 J+4 L)\right] x_{0}^{2} x_{2} \\
& +D I^{2}[D E(4 J+7 L)+F \omega L(2 J+L)+D F \omega(J+2 L)] x_{0} x_{2}^{2}+2 D^{4} J(E+\omega F) x_{0}+2 D^{4} I L x_{2} \\
& +D^{2} I[D(J+L)(5 E+3 \omega F)+2 F \omega J L] x_{0} x_{2}+\left(D^{2}+D E x_{0}+D I x_{2}+E I x_{0} x_{2}\right)\left(E J x_{0}-I L x_{2}\right)^{2} .
\end{aligned}
$$

Thus, $r_{1}>0$. Consequently, the conditions (C.8) are satisfied. Therefore, SS5 is stable if and only if $\lambda_{1}<0$. Since $M_{1}$ is increasing, this condition is equivalent to the stability condition of SS5 in Table 3.

For SS6, the characteristic polynomial is given by:

$$
P_{6}(\lambda)=\lambda^{6}+c_{1} \lambda^{5}+c_{2} \lambda^{4}+c_{3} \lambda^{3}+c_{4} \lambda^{2}+c_{5} \lambda+c_{6},
$$

where $c_{i}, i=1, \ldots, 6$ are defined in Table 2. From Lemma B.2, all of the roots of the sixth order polynomial have negative real parts if and only if $c_{i}>0, i=1,3,5,6$ and $r_{j}>0, j=4,5$, where $c_{i}$ and $r_{j}$ are listed in Table 2. Since $c_{1}$ and $c_{6}$ are positive, the proof is complete.

For SS7, the characteristic polynomial is

$$
P_{7}(\lambda)=\left(\lambda-\lambda_{1}\right)\left(\lambda-\lambda_{2}\right)(\lambda+D)^{2}\left(\lambda^{2}+c_{1} \lambda+c_{2}\right),
$$

where $\lambda_{1}=\mu_{0}\left(s_{0}^{\text {in }}, s_{1}^{\text {in }}-s_{1}+s_{2}^{\text {in }}\right)-D-a_{0}, \lambda_{2}=\mu_{2}\left(s_{1}^{\text {in }}-s_{1}+s_{2}^{\text {in }}\right)-D-a_{2}, c_{1}=$ $D+(G+H) x_{1}$ and $c_{2}=K(G+H) x_{1}$ where $s_{1}$ is the solution in the interval $J_{1}$ of equation $\psi_{1}\left(s_{1}\right)=D+a_{1}$. Since $c_{1}>0$ and $c_{2}>0$, the real parts of the roots of the quadratic factor are negative. Therefore, SS7 is stable if and only if $\lambda_{1}<0$ and $\lambda_{2}<0$. Since the functions $M_{2}$ and $M_{3}$ are increasing, the conditions $\lambda_{1}<0$ and $\lambda_{2}<0$ are equivalent to

$$
s_{1}>s_{1}^{\text {in }}+s_{2}^{\text {in }}-M_{3}\left(s_{0}^{\text {in }}, D+a_{0}\right) \quad \text { and } \quad s_{1}>s_{1}^{\text {in }}+s_{2}^{\text {in }}-M_{2}\left(D+a_{2}\right) .
$$


Since the function $\psi_{1}$ is increasing, (C.9) is equivalent to

$$
\psi_{1}\left(s_{1}\right)>\psi_{1}\left(s_{1}^{\text {in }}+s_{2}^{\text {in }}-M_{3}\left(s_{0}^{\text {in }}, D+a_{0}\right)\right), \quad \psi_{1}\left(s_{1}\right)>\psi_{1}\left(s_{1}^{\text {in }}+s_{2}^{\text {in }}-M_{2}\left(D+a_{2}\right)\right) .
$$

From the definition of the function $\psi_{1}$ together with the condition $\psi_{1}\left(s_{1}\right)=D+a_{1}$ which defines $s_{1}$, the preceding conditions are equivalent to

$$
\begin{array}{r}
\mu_{1}\left(s_{1}^{\text {in }}+s_{2}^{\text {in }}-M_{3}\left(s_{0}^{\text {in }}, D+a_{0}\right), M_{3}\left(s_{0}^{\text {in }}, D+a_{0}\right)\right)<D+a_{1}, \\
\mu_{1}\left(s_{1}^{\text {in }}+s_{2}^{\text {in }}-M_{2}\left(D+a_{2}\right), M_{2}\left(D+a_{2}\right)\right)<D+a_{1} .
\end{array}
$$

Since $M_{1}$ is increasing, these conditions are equivalent to the stability conditions of SS7 in Table 3.

For SS8, the characteristic polynomial is

$$
P_{8}(\lambda)=\left(\lambda-\lambda_{1}\right)(\lambda+D)\left(\lambda^{4}+c_{1} \lambda^{3}+c_{2} \lambda^{2}+c_{3} \lambda+c_{4}\right),
$$

where $\lambda_{1}=\mu_{0}\left(s_{0}^{\text {in }}, M_{2}\left(D+a_{2}\right)\right)-D-a_{0}$ and the coefficients $c_{i}$ are given by:

$$
\begin{aligned}
& c_{1}=2 D+(G+H) x_{1}+I x_{2}, \\
& c_{2}=D^{2}+(G+H)(D+K) x_{1}+I(D+L) x_{2}+G I x_{1} x_{2}, \\
& c_{3}=D(G+H) K x_{1}+D I L x_{2}+G I(K+L) x_{1} x_{2} \text { and } c_{4}=G I K L x_{1} x_{2} .
\end{aligned}
$$

From Lemma B.1, the roots of the fourth order polynomial are of negative real parts if and only if

$$
c_{i}>0, \quad \text { for } \quad i=1,3,4 \text { and } \quad r_{1}=c_{1} c_{2} c_{3}-c_{1}^{2} c_{4}-c_{3}^{2}>0 .
$$

We always have $c_{i}>0$, for $i=1,3,4$. We can write $r_{1}$ as follows:

$$
\begin{aligned}
r_{1}= & D K\left[(D+K)(G+H)^{3}-G^{3} K\right] x_{1}^{3}+D^{2} I^{3} L x_{2}^{3}+G^{2} I^{2}(G+H)(K+L) x_{1}^{3} x_{2}^{2}+G^{2} I^{3}(K+L) x_{1}^{2} x_{2}^{3} \\
& +G I\left[D(2 K+L)(G+H)^{2}+H K^{2}(2 G+H)\right] x_{1}^{3} x_{2}+D G I^{3}(K+2 L) x_{1} x_{2}^{3}+3 D^{3} I^{2} L x_{2}^{2} \\
& +D^{2} K\left[3 D(G+H)^{2}+H K(2 G+H)\right] x_{1}^{2}+G I^{2}\left[D(K+L)(5 G+3 H)+H\left(K^{2}+L^{2}\right)\right] x_{1}^{2} x_{2}^{2} \\
& +D I\left[H\left(D H(2 K+L)+H K(K+2 L)+D G(9 K+5 L)+2 G K^{2}\right)+D G^{2}(7 K+4 L)\right] x_{1}^{2} x_{2} \\
& +D I^{2}[D G(4 K+7 L)+H L(2 K+L)+D H(K+2 L)] x_{1} x_{2}^{2}+2 D^{4} K(G+H) x_{1}+2 D^{4} I L x_{2} \\
& +D^{2} I[D(K+L)(5 G+3 H)+2 H K L] x_{1} x_{2}+\left(D^{2}+D G x_{1}+D I x_{2}+G I x_{1} x_{2}\right)\left(G K x_{1}-I L x_{2}\right)^{2} .
\end{aligned}
$$

Thus, $r_{1}>0$. Consequently, the conditions (C.10) are satisfied. Finally, SS8 is stable if and only if $\lambda_{1}<0$, that is to say $\mu_{0}\left(s_{0}^{\text {in }}, M_{2}\left(D+a_{2}\right)\right)<D+a_{0}$. Since $M_{0}$ is increasing, this condition is equivalent to the stability condition of SS8 in Table 3.

C.2. Proof of Proposition 3.3. If SS2 exists then, its condition of existence $\mu_{2}\left(s_{2}^{\text {in }}\right)>D+a_{2}$ holds. Therefore, the condition $\mu_{2}\left(s_{2}^{\text {in }}\right)<D+a_{2}$ of stability of $\mathrm{SS} 1$ is not satisfied.

If SS3 exists then, its condition of existence $\mu_{0}\left(s_{0}^{\text {in }}, s_{2}^{\text {in }}\right)>D+a_{0}$ holds. Therefore, the condition $\mu_{0}\left(s_{0}^{\text {in }}, s_{2}^{\text {in }}\right)<D+a_{0}$ of stability of SS1 is not satisfied.

If SS7 exists then, its condition of existence $\mu_{1}\left(s_{1}^{\text {in }}, s_{2}^{\text {in }}\right)>D+a_{1}$ holds. Therefore, the condition $\mu_{1}\left(s_{1}^{\text {in }}, s_{2}^{\text {in }}\right)<D+a_{1}$ of stability of SS1 is not satisfied.

If SS6 exists then, the conditions

$$
(1-\omega) s_{0}^{\text {in }}+s_{1}^{\text {in }}+s_{2}^{\text {in }}>\phi_{2}(D), s_{0}^{\text {in }}>\varphi_{0}(D), s_{0}^{\text {in }}+s_{1}^{\text {in }}>\varphi_{0}(D)+\varphi_{1}(D)
$$


hold. Therefore, the condition $s_{0}^{\text {in }}<\varphi_{0}(D)$ of stability of SS2 or SS8 is not satisfied, the condition $(1-\omega) s_{0}^{\text {in }}+s_{1}^{\text {in }}+s_{2}^{\text {in }}<\phi_{2}(D)$ of stability of SS4 is not satisfied, and the condition $s_{0}^{\text {in }}+s_{1}^{\text {in }}<\varphi_{0}(D)+\varphi_{1}(D)$ of stability of SS5 is not satisfied.

If SS5 exists then, its conditions of existence

$$
s_{0}^{\text {in }}>\varphi_{0}(D) \quad \text { and } \quad s_{2}^{\text {in }}-\omega s_{0}^{\text {in }}>M_{2}\left(D+a_{2}\right)-\omega \varphi_{0}(D)
$$

hold. Therefore, the condition $s_{0}^{\text {in }}<\varphi_{0}(D)$ of stability of SS2 or SS8 is not satisfied and the condition $s_{2}^{\text {in }}-\omega s_{0}^{\text {in }}<M_{2}\left(D+a_{2}\right)-\omega \varphi_{0}(D)$ of stability of SS3 is not satisfied.

If SS8 exists then, its conditions of existence $s_{1}^{\text {in }}+s_{2}^{\text {in }}>\varphi_{1}(D)+M_{2}\left(D+a_{2}\right)$ holds. Therefore, the condition $s_{1}^{\text {in }}+s_{2}^{\text {in }}<\varphi_{1}(D)+M_{2}\left(D+a_{2}\right)$ of stability of SS7 is not satisfied.

Appendix D. Proof of Proposition 4.1. We assume that the biological parameter values in model (4.2) are provided in Table 15 . We assume that $S_{\mathrm{ph}}^{\mathrm{in}}=0$, $S_{\mathrm{H}_{2}}^{\mathrm{in}}=2.67 \times 10^{-5}$ as in Fig. $3(\mathrm{a})$ of [26]. We assume that $S_{\mathrm{ch}}^{\mathrm{in}}=0.1$. As said in Section 4, Theorem 3.1 applies to model (4.2). Using the change of variables (G.2) and Table 3, SS7 and SS8 do not exist when $S_{\mathrm{ph}}^{\text {in }}=0$. Moreover, the necessary and sufficient existence and stability conditions of steady states of (4.2) are summarized in Table 9.

TABLE 9

Existence and local stability conditions of steady states of $(4.2)$, when $S_{\mathrm{ph}}^{\mathrm{in}}=0$. The functions $\mu_{i}$ are given in (G.3) while $c_{3}, c_{5}, r_{4}$ and $r_{5}$ are defined in Table 2. All other functions are given in Table 8 and Table 16.

Existence conditions Stability conditions

\begin{tabular}{|c|c|c|}
\hline SS1 & Always exists & $\mu_{0}\left(Y S_{\mathrm{ch}}^{\text {in }}, S_{\mathrm{H}_{2}}^{\text {in }}\right)<D+a_{0}, \mu_{2}\left(S_{\mathrm{H}_{2}}^{\text {in }}\right)<D+a_{2}$ \\
\hline $\mathrm{SS} 2$ & $\mu_{2}\left(S_{\mathrm{H}_{2}}^{\mathrm{in}}\right)>D+a_{2}$ & $Y S_{\mathrm{ch}}^{\text {in }}<\varphi_{0}(D)$ \\
\hline SS3 & $\mu_{0}\left(Y S_{\mathrm{ch}}^{\mathrm{in}}, S_{\mathrm{H}_{2}}^{\mathrm{in}}\right)>D+a_{0}$ & $\begin{array}{l}\mu_{1}\left(Y S_{\mathrm{ch}}^{\mathrm{in}}-s_{0}, S_{\mathrm{H}_{2}}^{\mathrm{in}}-\omega\left(Y S_{\mathrm{ch}}^{\mathrm{in}}-s_{0}\right)\right)<D+a_{1} \\
S_{\mathrm{H}_{2}}^{\text {in }}-\omega Y S_{\mathrm{ch}}^{\mathrm{in}}<M_{2}\left(D+a_{2}\right)-\omega \varphi_{0}(D) \\
\text { with } s_{0} \text { solution of } \psi_{0}\left(s_{0}\right)=D+a_{0}\end{array}$ \\
\hline SS4 & $\begin{array}{l}(1-\omega) Y S_{\mathrm{ch}}^{\mathrm{in}}+S_{\mathrm{H}_{2}}^{\mathrm{in}} \geq \phi_{1}(D) \\
Y S_{\mathrm{ch}}^{\mathrm{in}}>M_{0}\left(D+a_{0}, s_{2}\right)+M_{1}\left(D+a_{1}, s_{2}\right) \\
\text { with } s_{2} \text { solution of } \\
\Psi\left(s_{2}, D\right)=(1-\omega) Y S_{\mathrm{ch}}^{\mathrm{in}}+S_{\mathrm{H}_{2}}^{\text {in }}\end{array}$ & $\begin{array}{l}(1-\omega) Y S_{\mathrm{ch}}^{\mathrm{in}}+S_{\mathrm{H}_{2}}^{\mathrm{in}}<\phi_{2}(D), \phi_{3}(D)>0 \\
\frac{\partial \Psi}{\partial s_{2}}\left(s_{2}, D\right)>0\end{array}$ \\
\hline SS5 & $\begin{array}{l}Y S_{\mathrm{ch}}^{\mathrm{in}}>\varphi_{0}(D) \\
S_{\mathrm{H}_{2}}^{\mathrm{in}}-\omega Y S_{\mathrm{ch}}^{\mathrm{in}}>M_{2}\left(D+a_{2}\right)-\omega \varphi_{0}(D)\end{array}$ & $Y S_{\mathrm{ch}}^{\mathrm{in}}<\varphi_{0}(D)+\varphi_{1}(D)$ \\
\hline SS6 & $\begin{array}{l}(1-\omega) Y S_{\mathrm{ch}}^{\mathrm{in}}+S_{\mathrm{H}_{2}}^{\mathrm{in}}>\phi_{2}(D) \\
Y S_{\mathrm{ch}}^{\mathrm{in}}>\varphi_{0}(D)+\varphi_{1}(D)\end{array}$ & $c_{3}>0, c_{5}>0, r_{4}>0, r_{5}>0$ \\
\hline
\end{tabular}

SS1 always exists and it is stable if and only if

$$
D>\mu_{0}\left(Y S_{\mathrm{ch}}^{\mathrm{in}}, S_{\mathrm{H}_{2}}^{\mathrm{in}}\right)-a_{0}:=\delta_{6} \quad \text { and } \quad D>\mu_{2}\left(S_{\mathrm{H}_{2}}^{\mathrm{in}}\right)-a_{2}:=\delta_{7} .
$$

Thus, SS1 is stable if and only if $D>\max \left(\delta_{6}, \delta_{7}\right)=\delta_{7}$ (see Table 4 for all critical parameter values $\left.\delta_{i}, i=1, \ldots, 7\right)$. From Table 9, SS2 exists if and only if $D<\delta_{7}$. From the eigenvalues $\lambda_{1}$ and $\lambda_{2}$ defined by (C.1), we deduce that SS2 is stable if and only if

$$
F_{1}(D):=\mu_{0}\left(Y S_{\mathrm{ch}}^{\mathrm{in}}, M_{2}\left(D+a_{2}\right)\right)-D-a_{0}<0 \quad \Longleftrightarrow \quad \delta_{5}<D<\delta_{7}
$$

where $\delta_{5}$ is the solution of equation $F_{1}(D)=0$ (see Figure D.1). SS3 exists if and 


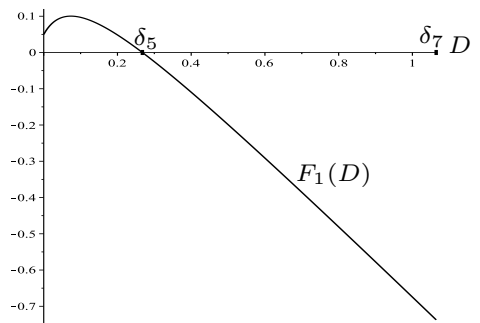

FIG. D.1. Stability of SS2 for all $D \in\left(\delta_{5}, \delta_{7}\right)$ : change of sign of the function $F_{1}(D)$.

only if $D<\delta_{6}$ and it is stable if and only if

$$
\begin{aligned}
& F_{2}(D):=\mu_{1}\left(S_{\mathrm{ch}}^{\mathrm{in}} Y-s_{0}, S_{\mathrm{H}_{2}}^{\mathrm{in}}-\omega\left(S_{\mathrm{ch}}^{\mathrm{in}} Y-s_{0}\right)\right)-D-a_{1}<0, \\
& F_{3}(D):=S_{\mathrm{H}_{2}}^{\mathrm{in}}+\omega\left(\varphi_{0}(D)-Y S_{\mathrm{ch}}^{\mathrm{in}}\right)-M_{2}\left(D+a_{2}\right)<0
\end{aligned}
$$

that is, $D<\delta_{4}$, where $\delta_{4}$ is the solution of equation $F_{3}(D)=0$ (see Figure D.2). From Remark 3.2, the system can have at most two steady states of the form SS4
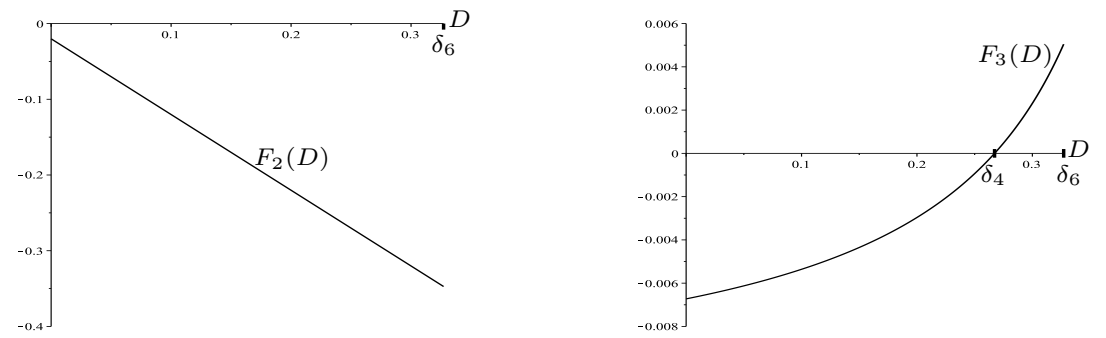

FIG. D.2. Stability of SS3 for all $D<\delta_{4}$ : signs of the functions $F_{2}(D)$ and $F_{3}(D)$.

denoted by $\mathrm{SS}^{1}$ and $\mathrm{SS} 4^{2}$ as $\omega \simeq 0.53<1$. Their first existence condition in Table 9 holds if and only if

$$
F_{4}(D):=\phi_{1}(D)-S_{\mathrm{H}_{2}}^{\text {in }}-(1-\omega) Y S_{\mathrm{ch}}^{\mathrm{in}} \leq 0 \quad \Longleftrightarrow \quad D \leq \delta_{3}
$$

where $\delta_{3}$ is the solution of equation $F_{4}(D)=0$ (see Figure D.3(a)). Their second existence condition holds for all $D \leq \delta_{3}$, since the straight line of equation $y=Y S_{\mathrm{ch}}^{\mathrm{in}}$ is above the curve of the function $y=M_{0}\left(D+a_{0}, s_{2}^{* i}\right)+M_{1}\left(D+a_{1}, s_{2}^{* i}\right)$, for $i=$ 1,2, which correspond to $\mathrm{SS}_{4}{ }^{1}$ and $\mathrm{SS}_{4}^{2}$, respectively, (see Figure D.3(b)). From
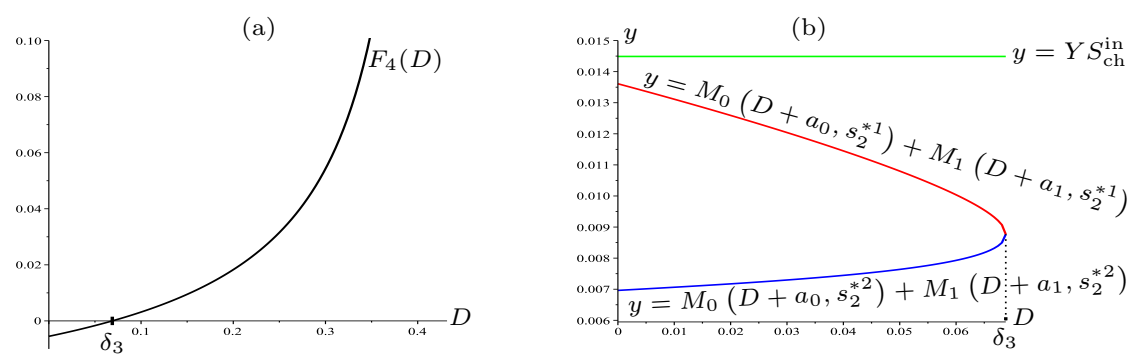

FIG. D.3. Existence of $S_{4} 4$ for all $D \leq \delta_{3}$ : (a) change of sign of the function $F_{4}(D)$, (b) the green line of equation $y=Y S_{\mathrm{ch}}^{\mathrm{in}}$ is above the red and blue curves of the functions $M_{0}\left(D+a_{0}, s_{2}^{* i}\right)+$ $M_{1}\left(D+a_{1}, s_{2}^{* i}\right), i=1,2$, respectively.

Remark 3.2 and Table $9, \mathrm{SS}^{1}$ is unstable for all $0<D<\delta_{3}$ while $\mathrm{SS}^{2}{ }^{2}$ is stable if 
and only if

$$
F_{5}(D):=\phi_{2}(D)-S_{\mathrm{H}_{2}}^{\mathrm{in}}-(1-\omega) Y S_{\mathrm{ch}}^{\mathrm{in}}>0 \quad \text { and } \quad \phi_{3}(D)>0
$$
Indeed, $F_{5}(D)>0$ for all $D \in\left(\delta_{2}, \delta_{3}\right)$ and $\phi_{3}(D)>0$ for all $D \in\left(\delta_{2}^{\prime}, \delta_{3}\right)$ where
$\delta_{2}^{\prime} \simeq 0.057865$ is the solution of equation $\phi_{3}(D)=0$ such that $\delta_{2}^{\prime}<\delta_{2}$.

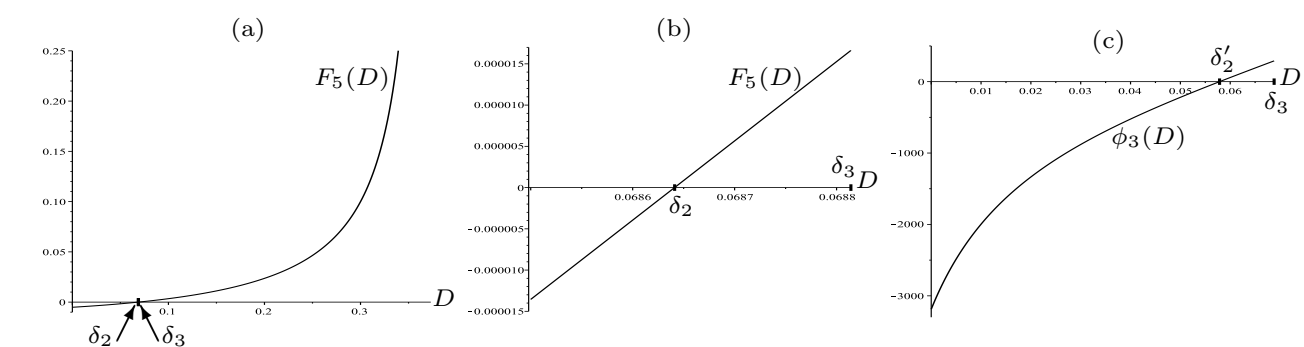

FIG. D.4. Stability of $S_{4}$ for all $D \in\left(\delta_{2}, \delta_{3}\right)$ : (a) Curve of the function $F_{5}(D)$. (b) Magnification of $F_{5}(D)$ for $D \in[0.0685,0.0688]$. (c) Curve of the function $\phi_{3}(D)$.

SS5 exists if and only if $F_{1}(D)>0$ and $F_{3}(D)>0$, that is, $\delta_{4}<D<\delta_{5}$. SS5 is stable if and only if

$$
F_{6}(D):=\varphi_{0}(D)+\varphi_{1}(D)-Y S_{\mathrm{ch}}^{\mathrm{in}}>0
$$

that is, for all $D \in\left(\delta_{4}, \delta_{5}\right)$ (see Figure D.5).
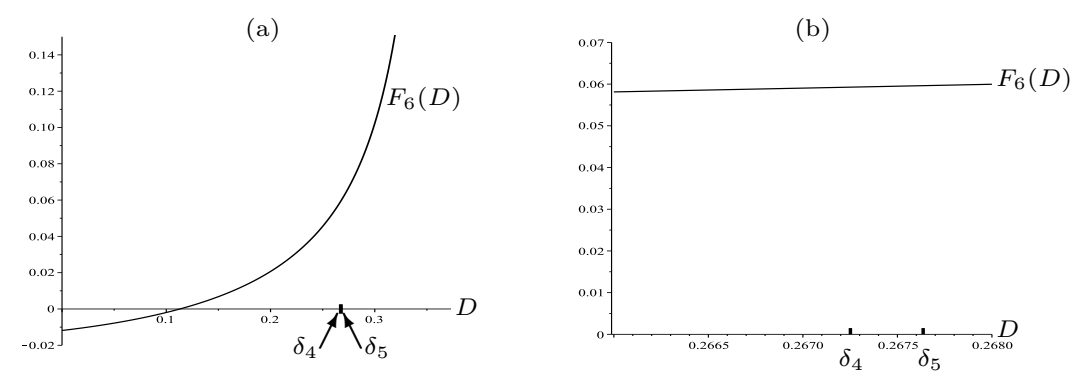

FIG. D.5. Stability of SS5 for all $D \in\left(\delta_{4}, \delta_{5}\right)$ and existence of SS6 for all $D<\delta_{2}$ : (a) curve of the function $F_{6}(D)$. (b) Magnification of $F_{6}(D)$ for $D \in[0.266,0.268]$.

SS6 exists if and only if $F_{5}(D)<0$ and $F_{6}(D)<0$, that is, for all $D<\delta_{2}$ where $\delta_{2}$ is the solution of the equation $F_{5}(D)=0$ (see Figure D.4(a-b) and Figure D.5). Indeed, $F_{5}(D)<0$ for all $D<\delta_{2}$ and $F_{6}(D)<0$ for all $D<\delta_{2}^{\prime \prime}$ where $\delta_{2}^{\prime \prime} \simeq 0.113033$ is the solution of equation $F_{6}(D)=0$ such that $\delta_{2}<\delta_{2}^{\prime \prime}$. To determine the stability of SS6, the functions $c_{3}, c_{5}, r_{4}$ and $r_{5}$ are plotted with respect to $D<\delta_{2}$. Figure D.6 shows that $c_{3}(D), c_{5}(D), r_{4}(D)$ and $r_{5}(D)$ are all positive if and only if $\delta_{1}<D<\delta_{2}$ where $\delta_{1} \simeq 0.010412$ is the solution of equation $r_{5}(D)=0$.

To give a numerical evidence of the Hopf bifurcation occurring for $D=\delta_{1}$, we determine numerically the eigenvalues of the Jacobian matrix of system (4.2) at SS6 and we plot them with respect to $D$. Figure D.7(a-b) shows that two eigenvalues denoted by $\lambda_{1}(D)$ and $\lambda_{2}(D)$ are real and remain negative for all $D \in\left[0, \delta_{2}\right)$. Figure D.7(c) shows that the two other eigenvalues $\lambda_{3}(D)$ and $\lambda_{4}(D)$ form a complex-conjugate pair denoted by

$$
\lambda_{3,4}(D)=\alpha_{3,4}(D) \pm i \beta_{3,4}(D), \quad \text { for all } \quad D \in\left[0, \delta^{\star}\right),
$$


(a)
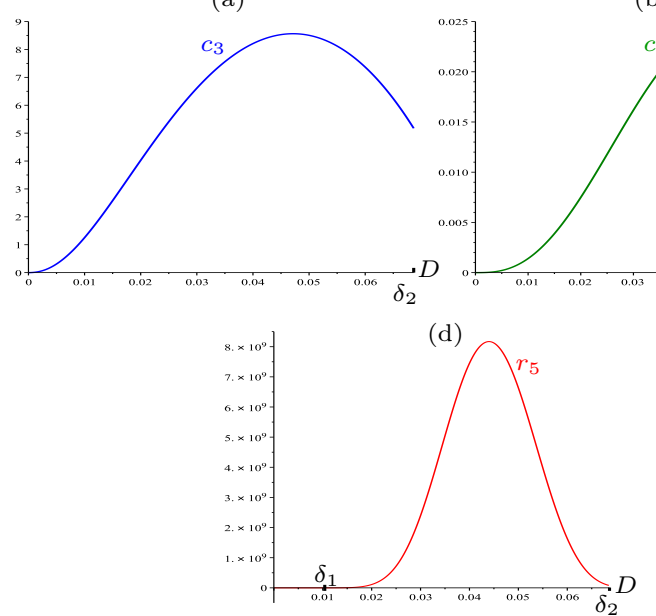

(b)

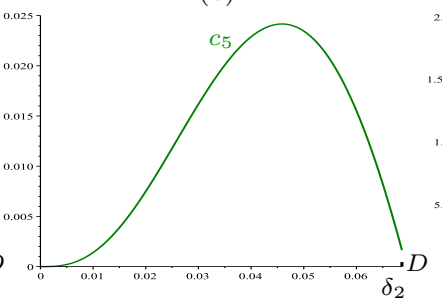

(c)

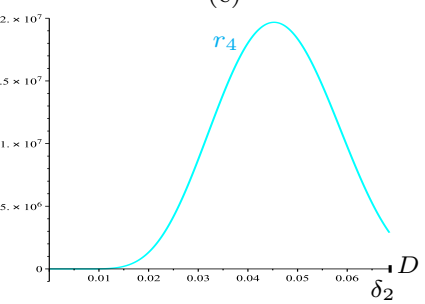

(e)

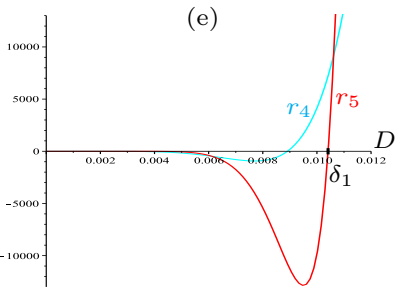

Fig. D.6. (a-b-c-d) Curves of the functions $c_{3}(D), c_{5}(D), r_{4}(D)$ and $r_{5}(D)$ for $0<D<\delta_{2}$. (e) Magnification of the curve of $r_{4}$ and $r_{5}$ for $D \in[0,0.02]$.

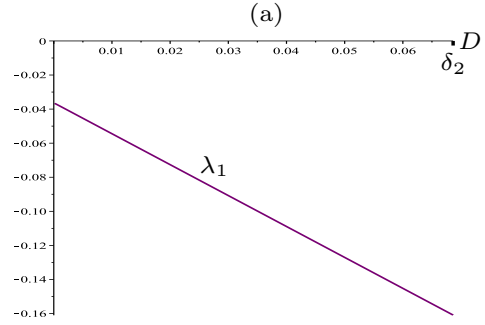

(c)

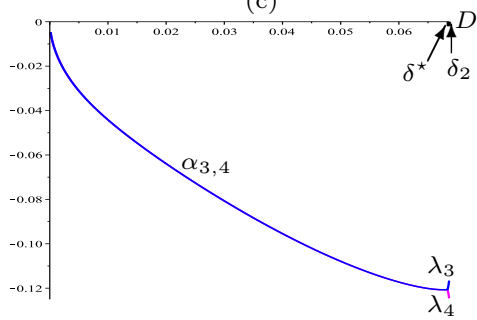

(b)

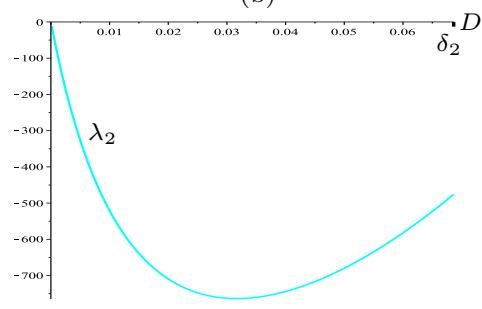

(d)

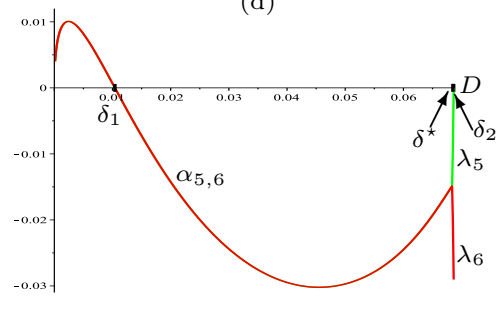

FIG. D.7. The eigenvalues of the Jacobian matrix at SS6 as a function of $D$, when $S_{\mathrm{ch}}^{\mathrm{in}}=0.1$, $S_{\mathrm{ph}}^{\mathrm{in}}=0$ and $S_{\mathrm{H}_{2}}^{\mathrm{in}}=2.67 \times 10^{-5}$. (c-d) The real parts $\alpha_{3,4}$ and $\alpha_{5,6}$ for $D \in\left[0, \delta^{\star}\right)$.

where the real part $\alpha_{3,4}$ remains negative and $\delta^{\star} \simeq 0.068504$. Then, they become real, negative and distinct for all $D \in\left(\delta^{\star}, \delta_{2}\right)$. Similarly, Figure D.7(d) shows that the two last eigenvalues $\lambda_{5}(D)$ and $\lambda_{6}(D)$ form a complex-conjugate pair denoted by

$$
\lambda_{5,6}(D)=\alpha_{5,6}(D) \pm i \beta_{5,6}(D), \quad \text { for all } D \in\left[0, \delta^{\star}\right),
$$

where the real part $\alpha_{5,6}$ is positive for all $D \in\left[0, \delta_{1}\right)$ and negative for all $D \in\left(\delta_{1}, \delta^{\star}\right)$. Then, for all $D \in\left(\delta^{\star}, \delta_{2}\right)$, they become real, negative and distinct. At the particular value $D=\delta_{1}$, the pair $\lambda_{5,6}(D)$ is purely imaginary such that $\alpha_{5,6}\left(\delta_{1}\right)=0$, with $\beta_{5,6}\left(\delta_{1}\right) \neq 0$. Moreover, one has

$$
\frac{d \alpha_{5,6}}{d D}\left(\delta_{1}\right)<0 .
$$

This is consistent with Figure 4.1(b) showing that, as $D$ decreases and crosses $\delta_{1}$, 
leading to the appearance, from the steady state SS6, of small-amplitude periodic oscillations.

Appendix E. Bifurcation diagram with respect to $S_{\mathrm{ch}}^{\mathrm{in}}$. In the following, we consider $S_{\mathrm{ph}}^{\mathrm{in}}=0$ and $S_{\mathrm{H}_{2}}^{\text {in }}=2.67 \times 10^{-5}$, corresponding to Fig. 3(a) in [26] and we fix $D=0.01$. Then, we determine the bifurcation diagram, where the input concentration $S_{\mathrm{ch}}^{\text {in }}$ is the bifurcation parameter. This choice for the operating parameters is identical to that in [16] excepted that we have added the microbial decay terms, as in [26]. Our aim is to compare our results to those of [26] and to see if there are interesting phenomena that were not detected in the operating diagram depicted in Fig. 3(a) of [26], see Remark E.2. Our aim is also to see the effects of mortality on the behavior of the process and to compare our bifurcation diagram to the one depicted in [16], see Remark E.3 below. Using Theorem 3.1, we have the following result, which is supported by numerical experimentation and is proved in Appendix F.

Proposition E.1. Let $S_{\mathrm{ph}}^{\mathrm{in}}=0, S_{\mathrm{H}_{2}}^{\mathrm{in}}=2.67 \times 10^{-5}$ and $D=0.01$. In this case, SS7 and SS8 do not exist. Using the biological parameter values in Table 15, the bifurcation values $\sigma_{i}, i=1, \ldots, 6$ are provided in Table 10. The bifurcation analysis of (4.2) according to $S_{\mathrm{ch}}^{\text {in }}$ is given in Table 11. The bifurcation types at the critical values $\sigma_{i}$ are defined in Table 12 .

TABLE 10

Critical parameter values $\sigma_{i}$, for $i=1, \ldots, 6$ where $Y$ is defined in Appendix $\mathrm{G}, r_{5}$ in Table 2 while all other functions are given in Table 8. Note that $\sigma_{1}<\sigma_{3}<\sigma_{4}<\sigma_{2}<\sigma_{5}<\sigma_{6}$, compare with Table 5 in [16].

\begin{tabular}{ll} 
Definition & Value \\
\hline$\sigma_{1}=M_{0}\left(D+a_{0}, S_{\mathrm{H}_{2}}^{\text {in }}\right) / Y$ & 0.003173 \\
$\sigma_{2}=\left(\phi_{1}(D)-S_{\mathrm{H}_{2}}^{\mathrm{in}}\right) /((1-\omega) Y)$ & 0.029402 \\
$\sigma_{3}=\varphi_{0}(D) / Y$ & 0.013643 \\
$\sigma_{4}=\left(S_{\mathrm{H}_{2}}^{\text {in }}-M_{2}\left(D+a_{2}\right)+\omega \varphi_{0}(D)\right) /(\omega Y)$ & 0.013985 \\
$\sigma_{5}=\left(\phi_{2}(D)-S_{\mathrm{H}_{2}}^{\mathrm{in}}\right) /((1-\omega) Y)$ & 0.033292 \\
$\sigma_{6}$ is the largest root of equation $r_{5}=0$ & 0.1025 \\
\hline
\end{tabular}

TABLE 11

Existence and stability of steady states, with respect to $S_{\mathrm{ch}}^{\mathrm{in}}$. The bifurcation values $\sigma_{i}, i=$ $1, \ldots, 6$ are given in Table 10.

\begin{tabular}{l|lllllll} 
Interval & $\mathrm{SS} 1$ & $\mathrm{SS} 2$ & $\mathrm{SS} 3$ & $\mathrm{SS}^{1}$ & $\mathrm{SS}^{2}$ & $\mathrm{SS} 5$ & $\mathrm{SS} 6$ \\
\hline $0<S_{\mathrm{ch}}^{\text {in }}<\sigma_{1}$ & $\mathrm{U}$ & $\mathrm{S}$ & & & & & \\
$\sigma_{1}<S_{\mathrm{ch}}^{\text {in }}<\sigma_{3}$ & $\mathrm{U}$ & $\mathrm{S}$ & $\mathrm{U}$ & & & & \\
$\sigma_{3}<S_{\mathrm{ch}}^{\text {in }}<\sigma_{4}$ & $\mathrm{U}$ & $\mathrm{U}$ & $\mathrm{U}$ & & & $\mathrm{S}$ & \\
$\sigma_{4}<S_{\mathrm{ch}}^{\text {in }}<\sigma_{2}$ & $\mathrm{U}$ & $\mathrm{U}$ & $\mathrm{S}$ & & & & \\
$\sigma_{2}<S_{\mathrm{ch}}^{\text {in }}<\sigma_{5}$ & $\mathrm{U}$ & $\mathrm{U}$ & $\mathrm{S}$ & $\mathrm{U}$ & $\mathrm{U}$ & & \\
$\sigma_{5}<S_{\mathrm{ch}}^{\text {in }}<\sigma_{6}$ & $\mathrm{U}$ & $\mathrm{U}$ & $\mathrm{S}$ & $\mathrm{U}$ & $\mathrm{U}$ & & $\mathrm{U}$ \\
$\sigma_{6}<S_{\mathrm{ch}}^{\text {in }}$ & $\mathrm{U}$ & $\mathrm{U}$ & $\mathrm{S}$ & $\mathrm{U}$ & $\mathrm{U}$ & & $\mathrm{S}$
\end{tabular}

Remark E.2. As explained in Remark 4.2, the operating diagram of Fig. 3(a) in [26] for $D=0.01$ does not accurately describe the transition from the region labeled SS2 (corresponding to the stability of SS2) to the SS3 region (corresponding to the stability of SS3). Our results show that this transition is via a SS5 region, which is very thin, since it corresponds to $\sigma_{3}<S_{\mathrm{ch}}^{\text {in }}<\sigma_{4}$, where $\sigma_{3} \simeq 0.013643$ and $\sigma_{4} \simeq 0.013985$. 
TABLE 12

Bifurcation types corresponding to the critical values of $\sigma_{i}, i=1, \ldots, 6$, defined in Table 10 . There exists also a critical value $\sigma^{*} \simeq 0.099295 \in\left(\sigma_{5}, \sigma_{6}\right)$ corresponding to the value of $S_{\mathrm{ch}}^{\mathrm{in}}$ where the stable limit cycle disappears when $S_{\mathrm{ch}}^{\mathrm{in}}$ is decreasing.

\section{Bifurcation types}

\begin{tabular}{ll}
\hline$\sigma_{1}$ & Transcritical bifurcation of SS1 and SS3 \\
$\sigma_{2}$ & Saddle-node bifurcation of SS4 ${ }^{1}$ and SS4 \\
$\sigma_{3}$ & Transcritical bifurcation of SS2 and SS5 \\
$\sigma_{4}$ & Transcritical bifurcation of SS3 and SS5 \\
$\sigma_{5}$ & Transcritical bifurcation of SS4 ${ }^{1}$ and SS6 \\
$\sigma_{6}$ & Supercritical Hopf bifurcation \\
$\sigma^{*}$ & Disappearance of the stable limit cycle
\end{tabular}

This region was missing in Fig. 3(a) in [26], since $\sigma_{4}-\sigma_{3}$ is of order $10^{-4}$. Indeed, the limitations of the operating diagram in Fig. 3(a) in [26] are due to the numerical resolution: the stability of SS5 occurs in a very small region and may not be detected if the step size was for example an order of magnitude greater than $\sigma_{4}-\sigma_{3}$.

Figures E.1 and E.2 show the one-parameter bifurcation diagrams of $X_{\mathrm{ch}}$ and $X_{\mathrm{H}_{2}}$ versus $S_{\mathrm{ch}}^{\text {in }}$ in system (4.2), respectively. The magnifications of the bifurcation diagrams are illustrated in Figure E.1(b), Figure E.2(b) and Figure E.3 showing the transcritical bifurcations at $\sigma_{1}, \sigma_{3}, \sigma_{4}$ and $\sigma_{5}$, the saddle-node bifurcation at $\sigma_{2}$, the Hopf bifurcation at $\sigma_{6}$ and the disappearance of the cycle at $\sigma^{*}$. In Figure E.1(b), SS1 and SS2 cannot be distinguished since they have both a zero $X_{\mathrm{ch}}$-component. As SS2 is stable and SS1 is unstable for $S_{\mathrm{ch}}^{\mathrm{in}}<\sigma_{3}$, the $X_{\mathrm{ch}}=0$ axis is plotted in blue as the color of SS2 in Table 7. In Figure E.2(b), SS1 and SS2 are distinguished but it is not the case for SS1 and SS3, since they have both a zero $X_{\mathrm{H}_{2}}$-component. As SS3 is stable and SS1 is unstable for $S_{\mathrm{ch}}^{\mathrm{in}}>\sigma_{4}$, the $X_{\mathrm{H}_{2}}=0$ axis is plotted in purple as the color of SS3 in Table 7.
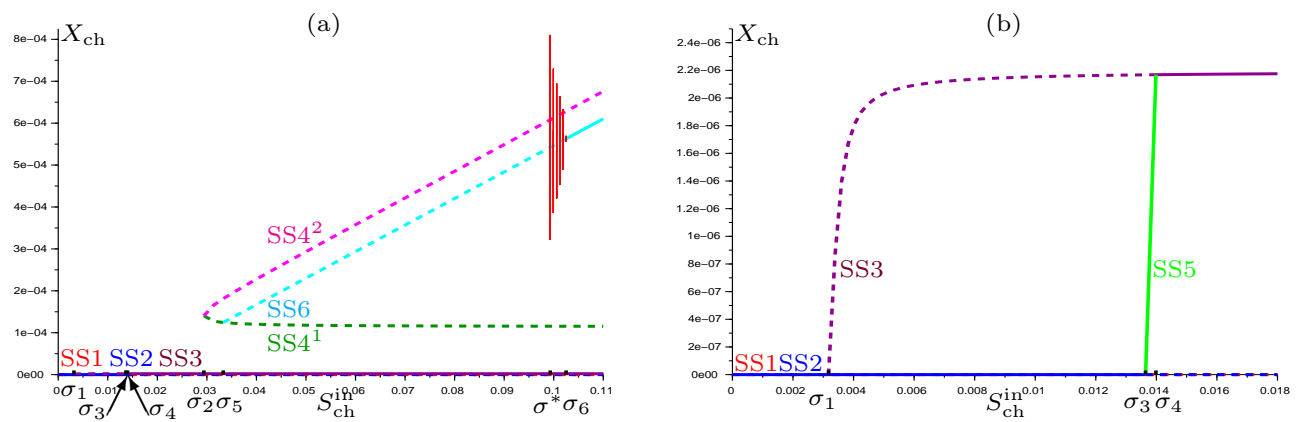

FIG. E.1. (a) Bifurcation diagram of $X_{\mathrm{ch}}$ versus $S_{\mathrm{ch}}^{\mathrm{in}} \in[0,0.11]$ in model (4.2) showing the appearance and disappearance of stable limit cycles. (b) Magnification on the transcritical bifurcations for $S_{\mathrm{ch}}^{\mathrm{in}} \in[0,0.018]$.

Remark E.3. As explained in Remark 3.2, with the exception of SS6, the maintenance does not destabilize the steady states. Only their regions of existence and stability, with respect to the operating parameters, can be modified. For SS6, it is more difficult to answer the question of whether or not it can be destabilized by including maintenance terms. The bifurcations diagrams depicted in Figures E.1 to E.3, and the results given in Proposition E.1, permit to answer this question at least for the following values of the operating parameters $S_{\mathrm{ph}}^{\text {in }}=0, S_{\mathrm{H}_{2}}^{\text {in }}=2.67 \times 10^{-5}, D=0.01$ 

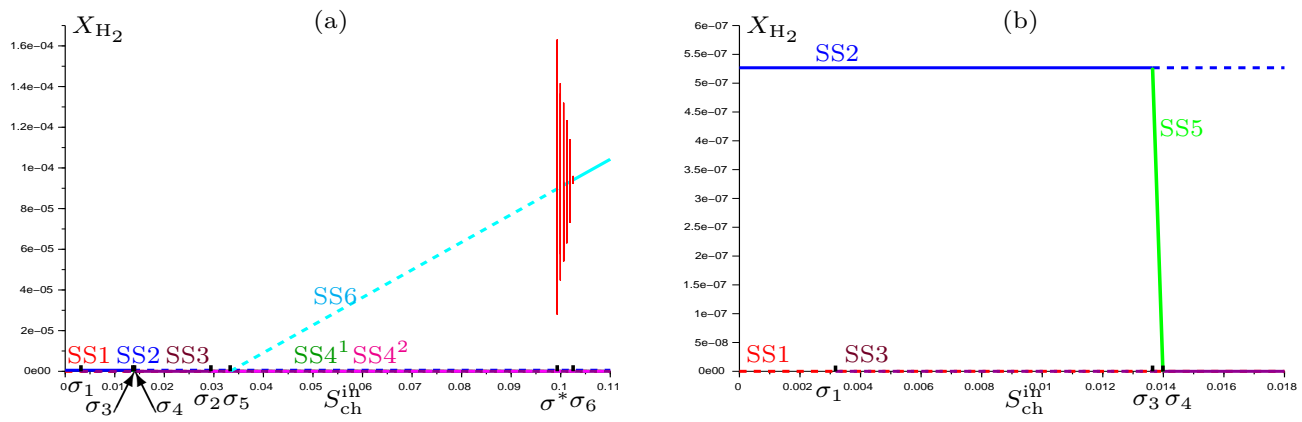

FIG. E.2. (a) Bifurcation diagram of $X_{\mathrm{H}_{2}}$ versus $S_{\mathrm{ch}}^{\mathrm{in}} \in[0,0.11]$ in model (4.2). (b) Magnification on the transcritical bifurcations for $S_{\mathrm{ch}}^{\mathrm{in}} \in[0,0.018]$.
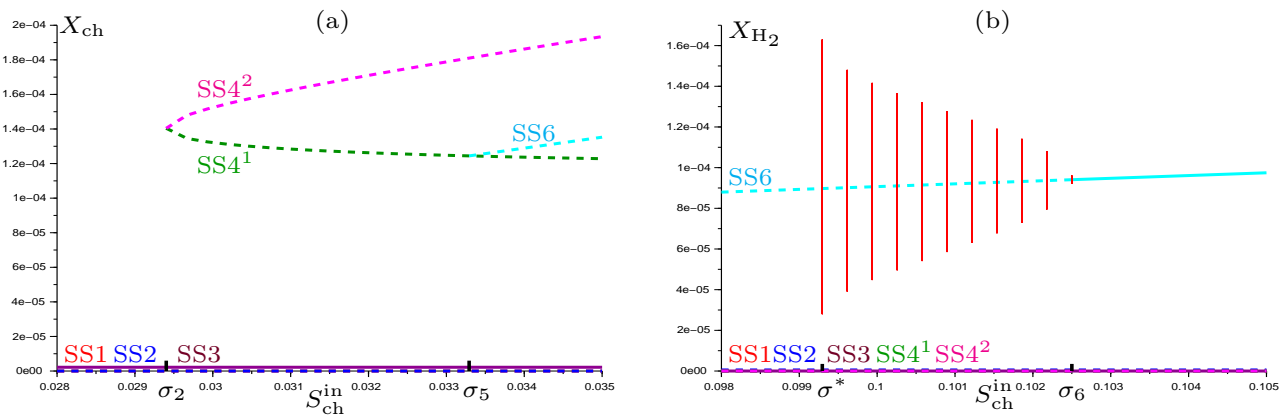

FIG. E.3. (a) Magnification on the saddle-node bifurcation at $S_{\mathrm{ch}}^{\mathrm{in}}=\sigma_{2}$ and the transcritical bifurcation at $S_{\mathrm{ch}}^{\mathrm{in}}=\sigma_{5}$ for $S_{\mathrm{ch}}^{\mathrm{in}} \in[0.028,0.035]$. (b) Magnification on the limit cycles for $S_{\mathrm{ch}}^{\mathrm{in}} \in$ $[0.098,0.105]$.

and $S_{\mathrm{ch}}^{\mathrm{in}} \geq 0$. The comparison of the results obtained in Table 11 with those given in Table 6 of [16] shows only minor changes in the bifurcation values $\sigma_{i}, i=1, \ldots, 6$. Therefore, even for SS6, the maintenance does not destabilize the system: only the regions of stability, with respect to the operating parameters, are slightly modified. Note that the change of the bifurcation values $\sigma_{i}$ is predictable since their formulas in Table 10 involve the added decay terms. However, the saddle-node bifurcation at $\sigma_{2}$ arises after and not before the transcritical bifurcations at $\sigma_{3}$ and $\sigma_{4}$ as in [16].

Appendix F. Proof of Proposition E.1. As said in Section 4, Theorem 3.1 applies to model (4.2). From Theorem 3.1 and the change of variables (G.2), SS7 and SS8 do not exist since $S_{\mathrm{ph}}^{\text {in }}=0$. The necessary and sufficient existence and stability conditions of all other steady states are summarized in Table 9. Since the second stability condition of SS1 in Table 9 does not hold

$$
\mu_{2}\left(S_{\mathrm{H}_{2}}^{\mathrm{in}}\right) \simeq 1.0845>D+a_{2}=0.03
$$

SS1 always exists and is unstable. Since the existence condition of SS2 in Table 9 holds (see inequality (F.1)), SS2 exists and is stable if and only if

$$
S_{\mathrm{ch}}^{\text {in }}<\varphi_{0}(D) / Y=: \sigma_{3} .
$$

SS3 exists if and only if

$$
S_{\mathrm{ch}}^{\text {in }}>M_{0}\left(D+a_{0}, S_{\mathrm{H}_{2}}^{\text {in }}\right) / Y=: \sigma_{1} .
$$


Let $F\left(S_{\mathrm{ch}}^{\mathrm{in}}\right)$ be the function defined by

The first stability condition of SS3 in Table 9 holds for all $S_{\mathrm{ch}}^{\mathrm{in}}>\sigma_{1}$, that is, $F\left(S_{\mathrm{ch}}^{\mathrm{in}}\right)<$ $D+a_{1}$ since the maximum of $F$ is smaller than 0.0013 while $D+a_{1}=0.03$ (see Figure F.1). From the second stability condition in Table 9, SS3 is stable if and only if

$$
S_{\mathrm{ch}}^{\mathrm{in}}>\left(S_{\mathrm{H}_{2}}^{\mathrm{in}}-M_{2}\left(D+a_{2}\right)+\omega \varphi_{0}(D)\right) /(\omega Y)=: \sigma_{4} .
$$
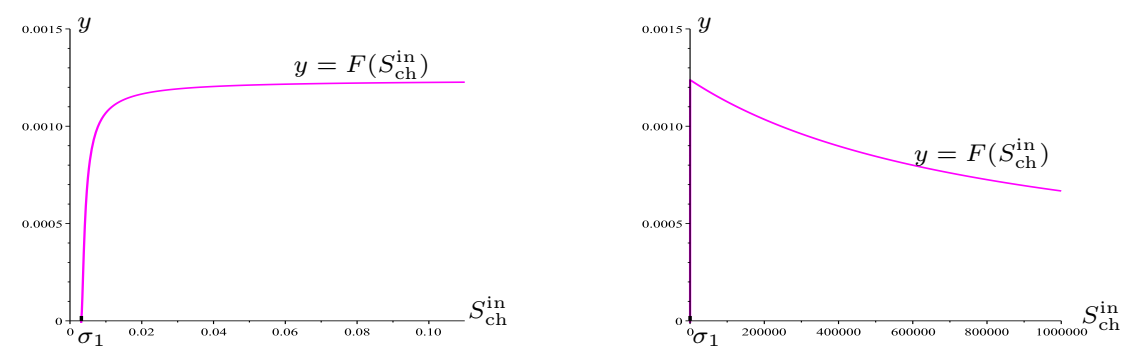

FIG. F.1. Curve of the function $y=F\left(S_{\mathrm{ch}}^{\mathrm{in}}\right)$ defined by (F.2).

From Theorem 3.1, the system can have at most two steady states of the form $\mathrm{SS} 4$ denoted by $\mathrm{SS}^{1}$ and $\mathrm{SS}^{2}$ as $\omega \simeq 0.53<1$. Their first existence condition in Table 9 holds if and only if

$$
S_{\mathrm{ch}}^{\mathrm{in}} \geq\left(\phi_{1}(D)-S_{\mathrm{H}_{2}}^{\mathrm{in}}\right) /((1-\omega) Y)=: \sigma_{2} .
$$

Their second existence condition holds, for all $S_{\mathrm{ch}}^{\mathrm{in}} \in\left[\sigma_{2}, 0.11\right]$, since the straight line of equation $y=S_{\mathrm{ch}}^{\mathrm{in}} Y$ is above the curves of the functions $y=M_{0}\left(D+a_{0}, s_{2}^{* i}\right)+$ $M_{1}\left(D+a_{1}, s_{2}^{* i}\right)$, for $i=1,2$, respectively (see Figure F.2). Thus, SS4 ${ }^{1}$ and SS4 ${ }^{2}$ exist and are unstable for all $S_{\mathrm{ch}}^{\mathrm{in}}>\sigma_{2}$ since the second stability condition does not hold where $\phi_{3}(D) \simeq-1996.917<0$.

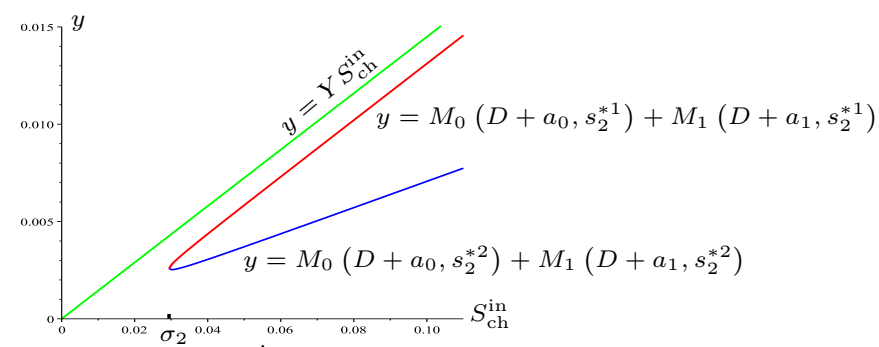

FIG. F.2. The green line of equation $y=Y S_{\mathrm{ch}}^{\mathrm{in}}$ is above the red and blue curves of the functions $M_{0}\left(D+a_{0}, s_{2}^{* i}\right)+M_{1}\left(D+a_{1}, s_{2}^{* i}\right)$, for $i=1,2$, which correspond to $\mathrm{SS}^{1}$ and $\mathrm{SS}^{2}$, respectively.

SS5 exists if and only if $\sigma_{3}:=\varphi_{0}(D) / Y<S_{\mathrm{ch}}^{\mathrm{in}}<\sigma_{4}$. When it exists, SS5 is stable since

$$
S_{\mathrm{ch}}^{\text {in }}<\sigma_{4} \simeq 0.013985<\left(\varphi_{0}(D)+\varphi_{1}(D)\right) / Y \simeq 0.02304 .
$$

SS6 exists if and only if

$$
S_{\mathrm{ch}}^{\text {in }}>\frac{\phi_{2}(D)-S_{\mathrm{H}_{2}}^{\text {in }}}{(1-\omega) Y}=: \sigma_{5} \simeq 0.033292, \quad S_{\mathrm{ch}}^{\text {in }}>\frac{\varphi_{0}(D)+\varphi_{1}(D)}{Y} \simeq 0.02304
$$


(a)

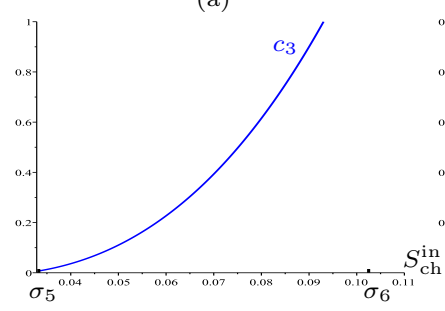

(d)

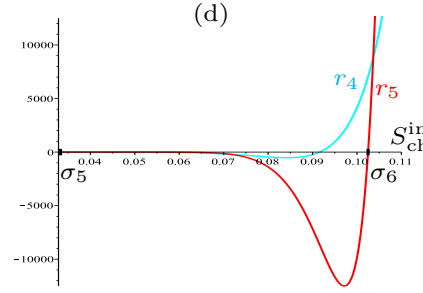

(b)

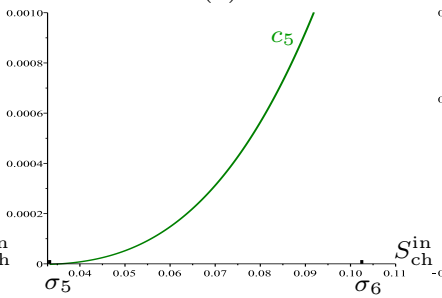

(e) $\quad S_{\text {in }}^{\text {in }}$

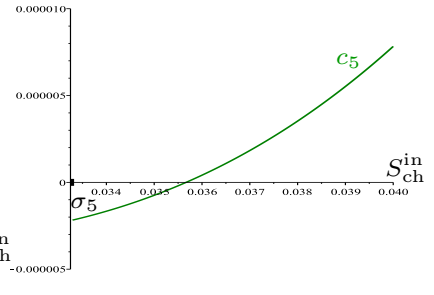

(f)

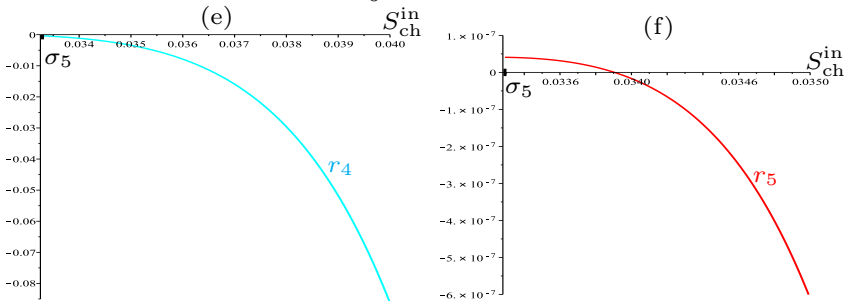

FIG. F.3. ( $a-b-d)$ Curves of the functions $c_{3}\left(S_{\mathrm{ch}}^{\mathrm{in}}\right), c_{5}\left(S_{\mathrm{ch}}^{\mathrm{in}}\right), r_{4}\left(S_{\mathrm{ch}}^{\mathrm{in}}\right)$ and $r_{5}\left(S_{\mathrm{ch}}^{\mathrm{in}}\right)$ for $S_{\mathrm{ch}}^{\mathrm{in}}>\sigma_{5}$. (c-e-f) Magnifications of the curves $c_{5}$ and $r_{4}$ for $S_{\mathrm{ch}}^{\mathrm{in}} \in\left[\sigma_{5}, 0.04\right]$ and of $r_{5}$ for $S_{\mathrm{ch}}^{\mathrm{in}} \in\left[\sigma_{5}, 0.035\right]$.
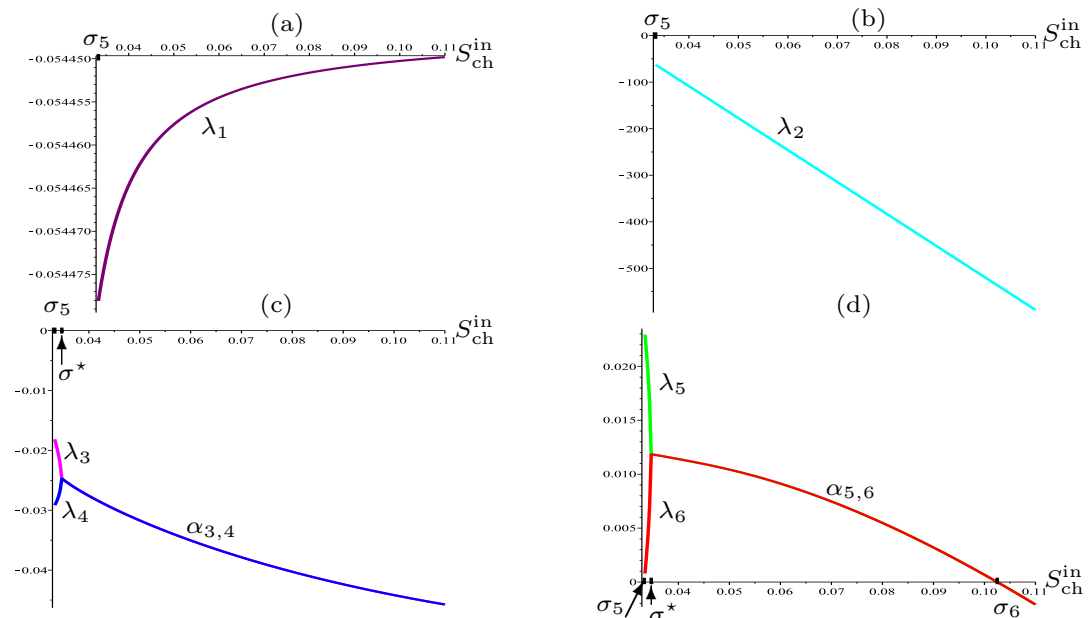

FIG. F.4. The eigenvalues of the Jacobian matrix at SS6 as a function of $S_{\mathrm{ch}}^{\mathrm{in}}$, when $D=0.01$, $S_{\mathrm{ph}}^{\mathrm{in}}=0$ and $S_{\mathrm{H}_{2}}^{\mathrm{in}}=2.67 \times 10^{-5}$. (c-d) The real parts $\alpha_{3,4}$ and $\alpha_{5,6}$ for $S_{\mathrm{ch}}^{\mathrm{in}} \in\left(\sigma^{\star}, 0.11\right]$.

Hence, SS6 exists if and only if $S_{\mathrm{ch}}^{\mathrm{in}}>\sigma_{5}$. To determine the stability of SS6, the functions $c_{3}, c_{5}, r_{4}$ and $r_{5}$ are plotted with respect to $S_{\mathrm{ch}}^{\text {in }}>\sigma_{5}$. Figure F.3 shows that $c_{3}\left(S_{\mathrm{ch}}^{\mathrm{in}}\right), c_{5}\left(S_{\mathrm{ch}}^{\mathrm{in}}\right), r_{4}\left(S_{\mathrm{ch}}^{\mathrm{in}}\right)$ and $r_{5}\left(S_{\mathrm{ch}}^{\mathrm{in}}\right)$ are all positive if and only if $S_{\mathrm{ch}}^{\mathrm{in}}>\sigma_{6}$ where $\sigma_{6} \simeq 0.1025$ is the largest root of equation $r_{5}\left(S_{\mathrm{ch}}^{\mathrm{in}}\right)=0$. To give a numerical evidence of the Hopf bifurcation occurring for $S_{\mathrm{ch}}^{\text {in }}=\sigma_{6}$, we determine numerically the eigenvalues of the Jacobian matrix of system (4.2) at SS6 and we plot them with respect to $S_{\mathrm{ch}}^{\mathrm{in}}$. Figure F.4(a-b) shows that two eigenvalues denoted by $\lambda_{1}\left(S_{\mathrm{ch}}^{\mathrm{in}}\right)$ and $\lambda_{2}\left(S_{\mathrm{ch}}^{\mathrm{in}}\right)$ are real and remain negative for all $S_{\mathrm{ch}}^{\text {in }} \in\left(\sigma_{5}, 0.11\right]$. Figure F.4(c) shows that the two other eigenvalues $\lambda_{3}\left(S_{\mathrm{ch}}^{\mathrm{in}}\right)$ and $\lambda_{4}\left(S_{\mathrm{ch}}^{\mathrm{in}}\right)$ are real, negative and distinct for all $S_{\mathrm{ch}}^{\text {in }} \in\left(\sigma_{5}, \sigma^{\star}\right)$ where $\sigma^{\star} \simeq 0.03467$. Then, they become a complex-conjugate pair denoted by

$$
\lambda_{3,4}\left(S_{\mathrm{ch}}^{\mathrm{in}}\right)=\alpha_{3,4}\left(S_{\mathrm{ch}}^{\mathrm{in}}\right) \pm i \beta_{3,4}\left(S_{\mathrm{ch}}^{\mathrm{in}}\right), \quad \text { for all } \quad S_{\mathrm{ch}}^{\mathrm{in}} \in\left(\sigma^{\star}, 0.11\right]
$$

where the real part $\alpha_{3,4}$ remains negative. 
Figure F.4(d) shows that the two last eigenvalues $\lambda_{5}\left(S_{\mathrm{ch}}^{\mathrm{in}}\right)$ and $\lambda_{6}\left(S_{\mathrm{ch}}^{\mathrm{in}}\right)$ are real, positive and distinct for all $S_{\mathrm{ch}}^{\text {in }} \in\left(\sigma_{5}, \sigma^{\star}\right]$. Then, they become a complex-conjugate pair denoted by

$$
\lambda_{5,6}\left(S_{\mathrm{ch}}^{\mathrm{in}}\right)=\alpha_{5,6}\left(S_{\mathrm{ch}}^{\mathrm{in}}\right) \pm i \beta_{5,6}\left(S_{\mathrm{ch}}^{\mathrm{in}}\right), \quad \text { for all } \quad S_{\mathrm{ch}}^{\mathrm{in}} \in\left(\sigma^{\star}, 0.11\right],
$$

so that the real part $\alpha_{5,6}$ is positive for all $S_{\mathrm{ch}}^{\mathrm{in}} \in\left(\sigma^{\star}, \sigma_{6}\right)$ and negative for all $S_{\mathrm{ch}}^{\mathrm{in}} \in$ $\left(\sigma_{6}, 0.11\right]$. At the particular value $S_{\mathrm{ch}}^{\mathrm{in}}=\sigma_{6}$, the pair $\lambda_{5,6}\left(S_{\mathrm{ch}}^{\mathrm{in}}\right)$ is purely imaginary such that $\alpha_{5,6}\left(\sigma_{6}\right)=0$, with $\beta_{5,6}\left(\sigma_{6}\right) \neq 0$. Moreover, one has

$$
\frac{d \alpha_{5,6}}{d S_{\mathrm{ch}}^{\text {in }}}\left(\sigma_{6}\right)<0 .
$$

This is consistent with Figures E.1 to E.3, showing that, as $S_{\mathrm{ch}}^{\mathrm{in}}$ decreases and crosses $\sigma_{6}$, the steady state SS6 changes its stability through a supercritical Hopf bifurcation with the emergence of a stable limit cycle that we illustrate in Figures 4.3 and H.1.

Remark F.1. Note that Figures F.1 and F.2 showing the stability of SS3 and the existence of two steady states of type SS4 are similar to Figures 6 and 7 in [16], respectively. But, on the contrary, Figure F.3 which concerns the stability of SS6 is completely different from Figure 8 in [16], since the conditions of stability of SS6 require to consider the signs of the Liénard-Chipart coefficients $c_{3}, c_{5}, r_{4}$ and $r_{5}$.

Appendix G. A chlorophenol-mineralising three-tiered microbial 'food web'. Following [20], model (4.2) can be written in the form of model (1.1), using the following change of variables:

$$
x_{0}=\frac{Y}{Y_{0}} X_{\mathrm{ch}}, x_{1}=\frac{Y_{4}}{Y_{1}} X_{\mathrm{ph}}, x_{2}=\frac{1}{Y_{2}} X_{\mathrm{H}_{2}}, s_{0}=Y S_{\mathrm{ch}}, s_{1}=Y_{4} S_{\mathrm{ph}}, s_{2}=S_{\mathrm{H}_{2}},
$$

where $Y=Y_{3} Y_{4}$. The input concentrations are given by:

$$
s_{0}^{\text {in }}=Y S_{\mathrm{ch}}^{\mathrm{in}}, \quad s_{1}^{\mathrm{in}}=Y_{4} S_{\mathrm{ph}}^{\mathrm{in}}, \quad s_{2}^{\text {in }}=S_{\mathrm{H}_{2}}^{\mathrm{in}},
$$

the death rates are $a_{0}=k_{\mathrm{dec}, \mathrm{ch}}, a_{1}=k_{\mathrm{dec}, \mathrm{ph}}, a_{2}=k_{\mathrm{dec}, \mathrm{H}_{2}}$, and the yield coefficients are

$$
Y_{0}=Y_{\mathrm{ch}}, \quad Y_{1}=Y_{\mathrm{ph}}, \quad Y_{2}=Y_{\mathrm{H}_{2}}, \quad Y_{3}=224 / 208\left(1-Y_{0}\right), \quad Y_{4}=32 / 224\left(1-Y_{1}\right)
$$

with $\omega=\frac{16}{208 Y}=\frac{1}{2\left(1-Y_{\mathrm{ch}}\right)\left(1-Y_{\mathrm{ph}}\right)}$. The specific growth functions (4.1) become the following functions satisfying Hypotheses (H1) to (H8):

$$
\mu_{0}\left(s_{0}, s_{2}\right)=\frac{m_{0} s_{0}}{K_{0}+s_{0}} \frac{s_{2}}{L_{0}+s_{2}}, \quad \mu_{1}\left(s_{1}, s_{2}\right)=\frac{m_{1} s_{1}}{K_{1}+s_{1}} \frac{1}{1+s_{2} / K_{I}}, \quad \mu_{2}\left(s_{2}\right)=\frac{m_{2} s_{2}}{K_{2}+s_{2}},
$$

where

$$
\begin{aligned}
& m_{0}=Y_{0} k_{m, \mathrm{ch}}, \quad K_{0}=Y K_{S, \mathrm{ch}}, \quad L_{0}=K_{S, \mathrm{H}_{2}, \mathrm{c}}, \quad m_{1}=Y_{1} k_{m, \mathrm{ph}} \\
& K_{1}=Y_{4} K_{S, \mathrm{ph}}, \quad K_{I}=K_{I, \mathrm{H}_{2}}, \quad m_{2}=Y_{2} k_{m, \mathrm{H}_{2}}, \quad K_{2}=K_{S, \mathrm{H}_{2}} .
\end{aligned}
$$

For these specific kinetics (G.3), the various functions defined in Table 8 are listed in Table 16. Using the linear change of variable given by (G.1) and (G.2), the yield coefficients in (4.2) are normalized to one except one of them, which is equal to $\omega \simeq 0.53$, when the yield coefficients are those given in Table 15. Therefore, (4.2) is of the form (1.1), with $\omega<1$ and the results of our paper apply to (4.2).

The aim of this section is to give rigorous proofs for the results of [26] on existence and stability of the steady states of model (4.2). Notice that the results in [26] were 
given with respect to the dimensionless form (H.2) of (4.2) by using the variables (H.1) and the growth functions (H.3). The variables (H.1) are related to our variables (G.1) by the formulas

$x_{0}=X_{0} K_{0}, x_{1}=X_{1} K_{1}, x_{2}=X_{2} K_{2}, s_{0}=S_{0} K_{0}, s_{1}=S_{1} K_{1}, s_{2}=S_{2} K_{2}, t=\tau / m_{0}$.

Hence, results given in variables (H.1) can be easily translated into results given in variables (G.1) and vice versa.

From Theorem 3.1, the existence and stability of steady states of model (4.2) can be determine for the specific growth functions (G.3). Using the functions and notations given in Table 16, we have the following results:

$\mathrm{SS} 1=\left(0,0,0, s_{0}^{\text {in }}, s_{1}^{\text {in }}, s_{2}^{\text {in }}\right)$ always exists. It is stable if and only if

$$
\mu_{0}\left(s_{0}^{\text {in }}, s_{2}^{\text {in }}\right)<D+a_{0}, \quad \mu_{1}\left(s_{1}^{\text {in }}, s_{2}^{\text {in }}\right)<D+a_{1} \quad \text { and } \quad \mu_{2}\left(s_{2}^{\text {in }}\right)<D+a_{2} .
$$

These conditions are equivalent to the conditions of [26], section C1, given in terms of variables (H.1) and growth functions (H.3).

$\mathrm{SS} 2=\left(0,0, x_{2}, s_{0}, s_{1}, s_{2}\right)$ is given by:

$$
s_{0}=s_{0}^{\text {in }}, \quad s_{1}=s_{1}^{\text {in }}, \quad s_{2}=\frac{K_{2}\left(D+a_{2}\right)}{m_{2}-D-a_{2}}, \quad x_{2}=\frac{D}{D+a_{2}}\left(s_{2}^{\text {in }}-s_{2}\right) .
$$

It exists if and only if $s_{2}^{\text {in }}>s_{2}$, where $s_{2}$ is given by (G.4). It is stable if and only if

$$
\mu_{0}\left(s_{0}^{\text {in }}, s_{2}\right)<D+a_{0} \quad \text { and } \quad \mu_{1}\left(s_{1}^{\text {in }}, s_{2}\right)<D+a_{1} .
$$

Formulas (G.4) together with the conditions of existence and stability of SS2 were established in [26], section C2, using variables (H.1) and growth functions (H.3).

$\mathrm{SS} 3=\left(x_{0}, 0,0, s_{0}, s_{1}, s_{2}\right)$ is given by:

$$
x_{0}=\frac{D}{D+a_{0}}\left(s_{0}^{\text {in }}-s_{0}\right), \quad s_{1}=s_{1}^{\text {in }}+s_{0}^{\text {in }}-s_{0}, \quad s_{2}=s_{2}^{\text {in }}-\omega\left(s_{0}^{\text {in }}-s_{0}\right),
$$

where $s_{0}$ is a solution of equation

$$
\frac{m_{0} s_{0}\left(s_{2}^{\text {in }}-\omega\left(s_{0}^{\text {in }}-s_{0}\right)\right)}{\left(K_{0}+s_{0}\right)\left(L_{0}+s_{2}^{\text {in }}-\omega\left(s_{0}^{\text {in }}-s_{0}\right)\right)}=D+a_{0} .
$$

Notice that (G.6) is a quadratic equation. Only its solution in the interval

$$
J_{0}=\left[\max \left(0, s_{0}^{\text {in }}-s_{2}^{\text {in }} / \omega\right), s_{0}^{\text {in }}\right)
$$

is to be considered. SS3 exists if and only if the following condition holds

$$
\mu_{0}\left(s_{0}^{\text {in }}, s_{2}^{\text {in }}\right)>D+a_{0} .
$$

It is stable if and only if

$$
\begin{aligned}
& \mu_{1}\left(s_{0}^{\text {in }}-s_{0}+s_{1}^{\text {in }}, s_{2}^{\text {in }}-\omega\left(s_{0}^{\text {in }}-s_{0}\right)\right)<D+a_{1}, \\
& s_{2}^{\text {in }}-\omega s_{0}^{\text {in }}<M_{2}\left(D+a_{2}\right)-\omega M_{0}\left(D+a_{0}, M_{2}\left(D+a_{2}\right)\right),
\end{aligned}
$$

where $s_{0}$ is the solution in the interval $J_{0}$ of equation (G.6). Formulas (G.5) together with equation (G.6) giving $s_{0}$ and the stability condition (G.8) were established in [26], section C3, using variables (H.1) and growth functions (H.3). However, neither condition (G.7) of existence of SS3 nor the signs of other eigenvalues of the Jacobian 
matrix were explicitly established in [26], section C3, where the existence and stability of SS3 were checked only numerically by considering the roots of polynomials of degrees 2 and 3, respectively, see formulas (C.3) and (C.7) in [26].

$\mathrm{SS} 4=\left(x_{0}, x_{1}, 0, s_{0}, s_{1}, s_{2}\right)$ is given by:

$$
\begin{aligned}
& s_{0}=\frac{\left(D+a_{0}\right) K_{0}\left(L_{0}+s_{2}\right)}{m_{0} s_{2}-\left(D+a_{0}\right)\left(L_{0}+s_{2}\right)}, \quad s_{1}=\frac{\left(D+a_{1}\right) K_{1}\left(K_{I}+s_{2}\right)}{m_{1} K_{I}-\left(D+a_{1}\right)\left(K_{I}+s_{2}\right)}, \\
& x_{0}=\frac{D}{D+a_{0}}\left(s_{0}^{\text {in }}-s_{0}\right), \quad x_{1}=\frac{D}{D+a_{1}}\left(s_{0}^{\text {in }}-s_{0}+s_{1}^{\text {in }}-s_{1}\right),
\end{aligned}
$$

where $s_{2}$ is a solution of equation

$$
\begin{aligned}
& (1-\omega) \frac{\left(D+a_{0}\right) K_{0}\left(L_{0}+s_{2}\right)}{m_{0} s_{2}-\left(D+a_{0}\right)\left(L_{0}+s_{2}\right)}+\frac{\left(D+a_{1}\right) K_{1}\left(K_{I}+s_{2}\right)}{m_{1} K_{I}-\left(D+a_{1}\right)\left(K_{I}+s_{2}\right)}+s_{2} \\
& =(1-\omega) s_{0}^{\text {in }}+s_{1}^{\text {in }}+s_{2}^{\text {in }} .
\end{aligned}
$$

Notice that (G.10) reduces to a cubic equation in $s_{2}$. Only its solutions in the interval $\left(s_{2}^{0}, s_{2}^{1}\right)$ are to be considered. The steady states $\mathrm{SS}_{4}{ }^{1}$ and $\mathrm{SS} 4^{2}$ exist if and only if the following conditions hold

$$
s_{0}^{\text {in }}>s_{0}, \quad s_{0}^{\text {in }}+s_{1}^{\text {in }}>s_{0}+s_{1} \quad \text { and } \quad(1-\omega) s_{0}^{\text {in }}+s_{1}^{\text {in }}+s_{2}^{\text {in }} \geq \phi_{1}(D),
$$

where $s_{0}$ and $s_{1}$ are defined by (G.9). SS4 $4^{1}$ is unstable whenever it exists and SS4 ${ }^{2}$ is stable if and only if

$$
(1-\omega) s_{0}^{\text {in }}+s_{1}^{\text {in }}+s_{2}^{\text {in }}<\phi_{2}(D), \text { and } \phi_{3}(D)>0 .
$$

Here $\phi_{2}$ and $\phi_{3}$ are defined in Table 8. Formulas (G.9) together with equation (G.10) giving $s_{2}$ were established in [26], section C4, using variables (H.1) and growth functions (H.3). However, neither condition (G.11) of existence of SS4 nor its condition of stability (G.12) have been established explicitly in [26], section C4, where the existence and stability of SS4 were checked only numerically by considering the roots of a polynomial of degree 5 , see formula (C.20) in [26].

$\mathrm{SS} 5=\left(x_{0}, 0, x_{2}, s_{0}, s_{1}, s_{2}\right)$ is given by:

$$
\begin{aligned}
& s_{2}=\frac{\left(D+a_{2}\right) K_{2}}{m_{2}-D-a_{2}}, \quad s_{0}=\frac{\left(D+a_{0}\right) K_{0}\left(L_{0}+s_{2}\right)}{m_{0} s_{2}-\left(D+a_{0}\right)\left(L_{0}+s_{2}\right)}, \quad s_{1}=s_{0}^{\text {in }}-s_{0}+s_{1}^{\text {in }}, \\
& x_{0}=\frac{D}{D+a_{0}}\left(s_{0}^{\text {in }}-s_{0}\right), \quad x_{2}=\frac{D}{D+a_{2}}\left(s_{2}^{\text {in }}-s_{2}-\omega\left(s_{0}^{\text {in }}-s_{0}\right)\right) .
\end{aligned}
$$

It exists if and only if the following conditions hold

$$
s_{0}^{\text {in }}>s_{0}, \quad s_{2}^{\text {in }}-\omega s_{0}^{\text {in }}>s_{2}-\omega s_{0} .
$$

where $s_{0}$ and $s_{2}$ are given by (G.13). SS5 is stable if and only if

$$
s_{0}^{\text {in }}+s_{1}^{\text {in }}<M_{0}\left(D+a_{0}, M_{2}\left(D+a_{2}\right)\right)+M_{1}\left(D+a_{1}, M_{2}\left(D+a_{2}\right)\right) .
$$

Formulas (G.13) together with conditions (G.14) of existence and (G.15) of stability of SS5 were established in [26], section C5, using variables (H.1) and growth functions (H.3). However, the signs of other eigenvalues of the Jacobian matrix have been checked only numerically by considering the roots of a polynomial of degree 4 , see formula (C.31) in [26].

$\mathrm{SS} 6=\left(x_{0}, x_{1}, x_{2}, s_{0}, s_{1}, s_{2}\right)$ is given by:

$$
\begin{aligned}
& s_{2}=\frac{\left(D+a_{2}\right) K_{2}}{m_{2}-D-a_{2}}, \quad s_{0}=\frac{\left(D+a_{0}\right) K_{0}\left(L_{0}+s_{2}\right)}{m_{0} s_{2}-\left(D+a_{0}\right)\left(L_{0}+s_{2}\right)}, \quad s_{1}=\frac{\left(D+a_{1}\right) K_{1}\left(K_{I}+s_{2}\right)}{m_{1} K_{I}-\left(D+a_{1}\right)\left(K_{I}+s_{2}\right)}, \\
& x_{0}=\frac{D}{D+a_{0}}\left(s_{0}^{\text {in }}-s_{0}\right), \quad x_{1}=\frac{D}{D+a_{1}}\left(s_{0}^{\text {in }}-s_{0}+s_{1}^{\text {in }}-s_{1}\right), \\
& x_{2}=\frac{D}{D+a_{2}}\left((1-\omega)\left(s_{0}^{\text {in }}-s_{0}\right)+s_{1}^{\text {in }}-s_{1}+s_{2}^{\text {in }}-s_{2}\right) .
\end{aligned}
$$


It exists if and only if the following conditions hold

$$
s_{0}^{\text {in }}>s_{0}, \quad s_{0}^{\text {in }}+s_{1}^{\text {in }}>s_{0}+s_{1}, \quad(1-\omega) s_{0}^{\text {in }}+s_{1}^{\text {in }}+s_{2}^{\text {in }}>\phi_{2}(D),
$$

where $s_{0}$ and $s_{1}$ are given by (G.16). SS6 is stable if and only if

$$
c_{i}>0, \quad i=3,5, \quad \text { and } \quad r_{j}>0, \quad j=4,5,
$$

where $c_{i}$ and $r_{j}$ are defined in Table 2. Formulas (G.16) together with conditions (G.17) of existence of SS6 were established in [26], section C6, using variables (H.1) and growth functions (H.3). However, the Liénard-Chipart stability conditions (G.18) were not considered in [26], where the stability of SS6 was checked only numerically by considering the roots of a polynomial of degree 6, see formula (C.42) in [26].

$\mathrm{SS} 7=\left(0, x_{1}, 0, s_{0}, s_{1}, s_{2}\right)$ is given by:

$$
s_{0}=s_{0}^{\text {in }}, \quad x_{1}=\frac{D}{D+a_{1}}\left(s_{1}^{\text {in }}-s_{1}\right), \quad s_{2}=s_{1}^{\text {in }}-s_{1}+s_{2}^{\text {in }},
$$

where $s_{1}$ is a unique solution of equation

$$
\frac{m_{1} s_{1} K_{I}}{\left(K_{1}+s_{1}\right)\left(K_{I}+s_{1}^{\text {in }}+s_{2}^{\text {in }}-s_{1}\right)}=D+a_{1} .
$$

Notice that (G.20) is a quadratic equation. Only its solution in the interval

$$
J_{1}=\left[0, s_{1}^{\text {in }}\right)
$$

is to be considered. SS7 exists if and only if the following condition holds

$$
\mu_{1}\left(s_{1}^{\text {in }}, s_{2}^{\text {in }}\right)>D+a_{1} .
$$

SS7 is stable if and only if

$$
\begin{aligned}
& s_{1}^{\text {in }}+s_{2}^{\text {in }}<M_{1}\left(D+a_{1}, M_{3}\left(s_{0}^{\text {in }}, D+a_{0}\right)\right)+M_{3}\left(s_{0}^{\text {in }}, D+a_{0}\right), \\
& s_{1}^{\text {in }}+s_{2}^{\text {in }}<M_{1}\left(D+a_{1}, M_{2}\left(D+a_{2}\right)\right)+M_{2}\left(D+a_{2}\right) .
\end{aligned}
$$

Formulas (G.19) together with equation (G.20) giving $s_{1}$ and stability condition (G.22) were established in [26], section C7, using variables (H.1) and growth functions (H.3). However, condition (G.21) of existence of SS7 has not been established explicitly in [26], section C7, where the existence of SS7 and the signs of other eigenvalues of the Jacobian matrix were checked only numerically by considering the roots of a polynomial of degree 3 , see formula (C.53) in [26].

$\mathrm{SS} 8=\left(0, x_{1}, x_{2}, s_{0}, s_{1}, s_{2}\right)$ is given by:

$$
\begin{aligned}
& s_{0}=s_{0}^{\text {in }}, \quad s_{2}=\frac{\left(D+a_{2}\right) K_{2}}{m_{2}-D-a_{2}}, \quad s_{1}=\frac{\left(D+a_{1}\right) K_{1}\left(K_{I}+s_{2}\right)}{m_{1} K_{I}-\left(D+a_{1}\right)\left(K_{I}+s_{2}\right)}, \\
& x_{1}=\frac{D}{D+a_{1}}\left(s_{1}^{\text {in }}-s_{1}\right), \quad x_{2}=\frac{D}{D+a_{2}}\left(s_{1}^{\text {in }}-s_{1}+s_{2}^{\text {in }}-s_{2}\right) .
\end{aligned}
$$

It exists if and only if the following conditions hold

$$
s_{1}^{\text {in }}>s_{1}, \quad s_{1}^{\text {in }}+s_{2}^{\text {in }}>s_{1}+s_{2},
$$

where $s_{1}$ and $s_{2}$ are given by (G.23). SS8 is stable if and only if

$$
s_{0}^{\text {in }}<M_{0}\left(D+a_{0}, M_{2}\left(D+a_{2}\right)\right) .
$$


Formulas (G.23) together with conditions (G.24) of existence and (G.25) of stability of SS8 were established in [26], section C8, using variables (H.1) and growth functions (H.3). However, the signs of other eigenvalues of the Jacobian matrix have been checked only numerically by considering the roots of a polynomial of degree 4 , see formula (C.62) in [26].

Appendix H. Numerical simulations. The plots of Figures F.1 to F.4 were performed with Maple [11], which is used in particular for the computations of coefficients $c_{3}, c_{5}, r_{4}$ and $r_{5}$, evaluated at SS6, and the computations of the eigenvalues of the Jacobian matrix evaluated at SS6. The plots of Figures E.1 to E.3 were performed with Scilab [22] by using the formulas of the steady state components given in Table 1. The various functions appearing in these formulas are given in Table 16 . The plots of Figures 4.2 to 4.4 and H.1 to H.4 were performed with Scilab [22]. The numerical simulations presented in Figures 4.2 to 4.4, F.4, and H.1 to H.4 were performed on the dimensionless form of (4.2) used in [26]. Indeed, in the original form (4.2), numerical instabilities arise in numerical schemes. To reduce the number of parameters describing the dynamics and facilitate numerical simulations, the following rescaling of the variables was used in [26]:

$$
\begin{aligned}
& X_{0}=\frac{X_{\mathrm{ch}}}{K_{\mathrm{S}, \mathrm{ch}} Y_{\mathrm{ch}}}, \quad X_{1}=\frac{X_{\mathrm{ph}}}{K_{\mathrm{S}, \mathrm{ph}} Y_{\mathrm{ph}}}, \quad X_{2}=\frac{X_{\mathrm{H}_{2}}}{K_{\mathrm{S}, \mathrm{H}_{2}} Y_{\mathrm{H}_{2}}}, \\
& S_{0}=\frac{S_{\mathrm{ch}}}{K_{\mathrm{S}, \mathrm{ch}}}, \quad S_{1}=\frac{S_{\mathrm{ph}}}{K_{\mathrm{S}, \mathrm{ph}}}, \quad S_{2}=\frac{S_{\mathrm{H}_{2}}}{K_{\mathrm{S}, \mathrm{H}_{2}}}, \quad \tau=k_{\mathrm{m}, \mathrm{ch}} Y_{\mathrm{ch}} t .
\end{aligned}
$$

Then, with these changes of variables the system given in (4.2) reduced to system

$$
\left\{\begin{aligned}
\frac{d X_{0}}{d \tau} & =\left(\nu_{0}\left(S_{0}, S_{2}\right)-\alpha-k_{0}\right) X_{0} \\
\frac{d X_{1}}{d \tau} & =\left(\nu_{1}\left(S_{1}, S_{2}\right)-\alpha-k_{1}\right) X_{1} \\
\frac{d X_{2}}{d \tau} & =\left(\nu_{2}\left(S_{2}\right)-\alpha-k_{2}\right) X_{2} \\
\frac{d S_{0}}{d \tau} & =\alpha\left(u_{0}-S_{0}\right)-\nu_{0}\left(S_{0}, S_{2}\right) X_{0} \\
\frac{d S_{1}}{d \tau} & =\alpha\left(u_{1}-S_{1}\right)+\omega_{0} \nu_{0}\left(S_{0}, S_{2}\right) X_{0}-\nu_{1}\left(S_{1}, S_{2}\right) X_{1} \\
\frac{d S_{2}}{d \tau} & =\alpha\left(u_{2}-S_{2}\right)-\omega_{2} \nu_{0}\left(S_{0}, S_{2}\right) X_{0}+\omega_{1} \nu_{1}\left(S_{1}, S_{2}\right) X_{1}-\nu_{2}\left(S_{2}\right) X_{2}
\end{aligned}\right.
$$

The operating parameters are

$$
\alpha=\frac{D}{k_{\mathrm{m}, \mathrm{ch}} Y_{\mathrm{ch}}}, \quad u_{0}=\frac{S_{\mathrm{ch}}^{\mathrm{in}}}{K_{\mathrm{S}, \mathrm{ch}}}, \quad u_{1}=\frac{S_{\mathrm{ph}}^{\mathrm{in}}}{K_{\mathrm{S}, \mathrm{ph}}}, \quad u_{2}=\frac{S_{\mathrm{H}_{2}}^{\mathrm{in}}}{K_{\mathrm{S}, \mathrm{H}_{2}}} .
$$

The yield coefficients are

$$
\omega_{0}=\frac{K_{\mathrm{S}, \mathrm{ch}}}{K_{\mathrm{S}, \mathrm{ph}}} \frac{224}{208}\left(1-Y_{\mathrm{ch}}\right), \quad \omega_{1}=\frac{K_{\mathrm{S}, \mathrm{ph}}}{K_{\mathrm{S}, \mathrm{H}_{2}}} \frac{32}{224}\left(1-Y_{\mathrm{ph}}\right), \quad \omega_{2}=\frac{16}{208} \frac{K_{\mathrm{S}, \mathrm{ch}}}{K_{\mathrm{S}, \mathrm{H}_{2}}} .
$$

The death rates are

$$
k_{0}=\frac{k_{\mathrm{dec}, \mathrm{ch}}}{k_{\mathrm{m}, \mathrm{ch}} Y_{\mathrm{ch}}}, \quad k_{1}=\frac{k_{\mathrm{dec}, \mathrm{ph}}}{k_{\mathrm{m}, \mathrm{ch}} Y_{\mathrm{ch}}}, \quad k_{2}=\frac{k_{\mathrm{dec}, \mathrm{H}_{2}}}{k_{\mathrm{m}, \mathrm{ch}} Y_{\mathrm{ch}}} .
$$

The growth functions are

$$
\nu_{0}\left(S_{0}, S_{2}\right)=\frac{S_{0}}{1+S_{0}} \frac{S_{2}}{K_{P}+S_{2}}, \quad \nu_{1}\left(S_{1}, S_{2}\right)=\frac{\phi_{1} S_{1}}{1+S_{1}} \frac{1}{1+K_{I} S_{2}}, \quad \nu_{2}\left(S_{2}\right)=\frac{\phi_{2} S_{2}}{1+S_{2}},
$$

where the biological parameters are given by

$$
\phi_{1}=\frac{k_{\mathrm{m}, \mathrm{ph}} Y_{\mathrm{ph}}}{k_{\mathrm{m}, \mathrm{ch}} Y_{\mathrm{ch}}}, \quad \phi_{2}=\frac{k_{\mathrm{m}, \mathrm{H}_{2}} Y_{\mathrm{H}_{2}}}{k_{\mathrm{m}, \mathrm{ch}} Y_{\mathrm{ch}}}, \quad K_{P}=\frac{K_{\mathrm{S}, \mathrm{H}_{2}, \mathrm{C}}}{K_{\mathrm{S}, \mathrm{H}_{2}}}, \quad K_{I}=\frac{K_{\mathrm{S}, \mathrm{H}_{2}}}{K_{\mathrm{I}, \mathrm{H}_{2}}} .
$$



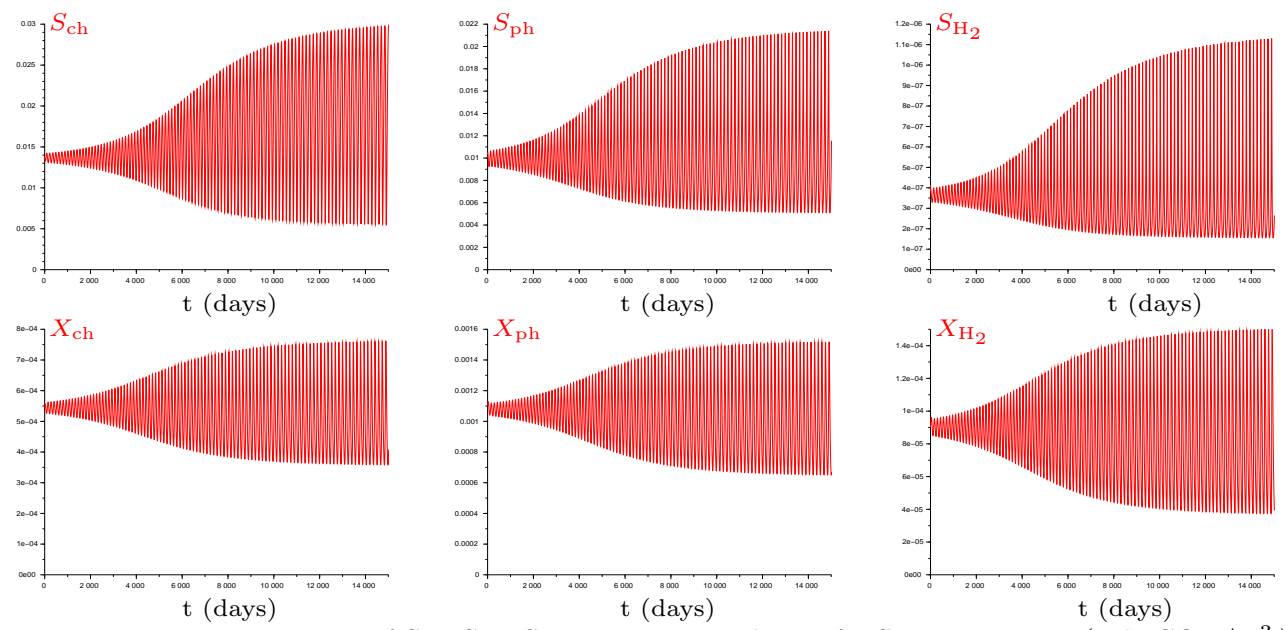

FIG. H.1. Trajectories of $S_{\mathrm{ch}}, S_{\mathrm{ph}}, S_{\mathrm{H}_{2}}, X_{\mathrm{ch}}, X_{\mathrm{ph}}$ and $X_{\mathrm{H}_{2}}$ for $\mathrm{S}_{\mathrm{ch}, \text { in }}=0.0995\left(\right.$ in $\left.\mathrm{kgCOD} / \mathrm{m}^{3}\right)$ : Convergence to the stable limit cycle.
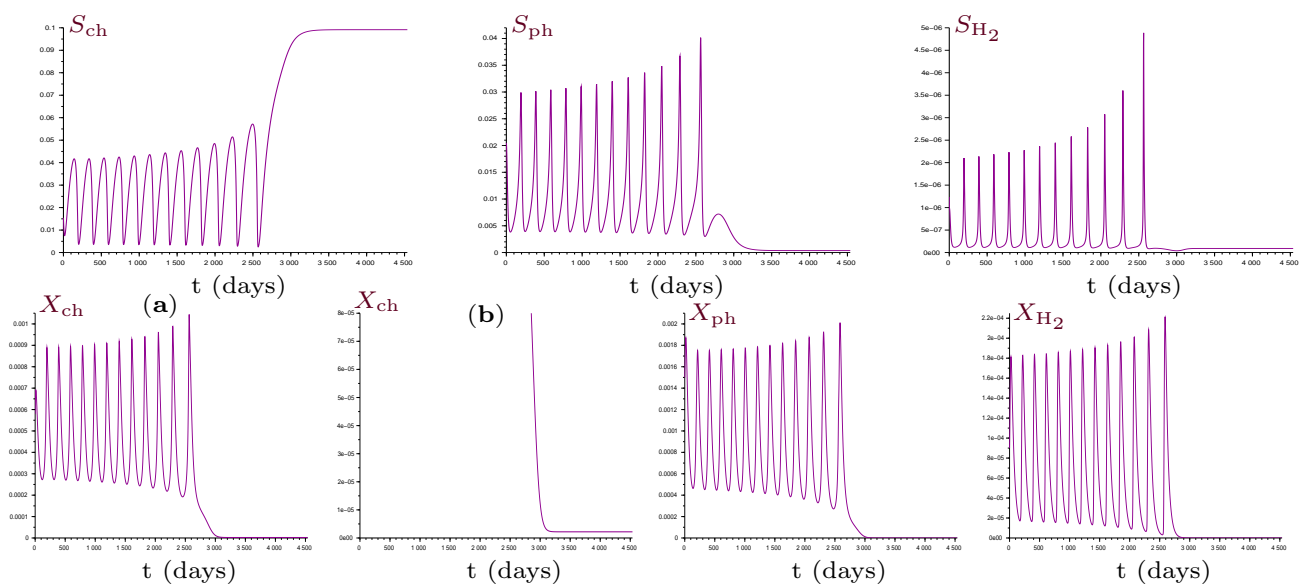

FIG. H.2. Trajectories of $S_{\mathrm{ch}}, S_{\mathrm{ph}}, S_{\mathrm{H}_{2}}, X_{\mathrm{ch}}, X_{\mathrm{ph}}$ and $X_{\mathrm{H}_{2}}$ for $\mathrm{S}_{\mathrm{ch}, \text { in }}=0.0995\left(\right.$ in $\left.\mathrm{kgCOD} / \mathrm{m}^{3}\right)$ : Convergence to the stable steady state SS3. (b) A magnification of (a) showing that the solution of (4.2) converges to the nonzero $X_{\mathrm{ch}}$-component of SS3.

The trajectories in Figures 4.2 to 4.4 and H.1 to H.4 were presented according to the variables of model (4.2) using the change of variables (H.1). In Figures 4.2 to 4.4, the projections of the orbits of the six-dimensional phase space into the threedimensional space $\left(X_{\mathrm{ch}}, X_{\mathrm{ph}}, X_{\mathrm{H}_{2}}\right)$ shows the appearance and disappearance of a stable limit cycle for different values of $S_{\mathrm{ch}}^{\mathrm{in}}>\sigma_{5}$. The plot of the limit cycle was obtained by solving the ordinary differential equations using the default solver "lsoda" from the ODEPACK package in Scilab. Tables 13 and 14 present the components of the stable steady states SS3 and SS6, and all the initial conditions chosen to trace the different trajectories of model (4.2) in Figures 4.2 to 4.4 and H.1 to H.4.

Appendix I. Tables. In this section, the biological parameter values are provided in Table 15. In Table 16, we present the auxiliary functions in the case of the growth functions given by (G.3).

Acknowledgments. The authors thank the Editor and the two anonymous reviewers for their constructive comments which have greatly improved this work. 

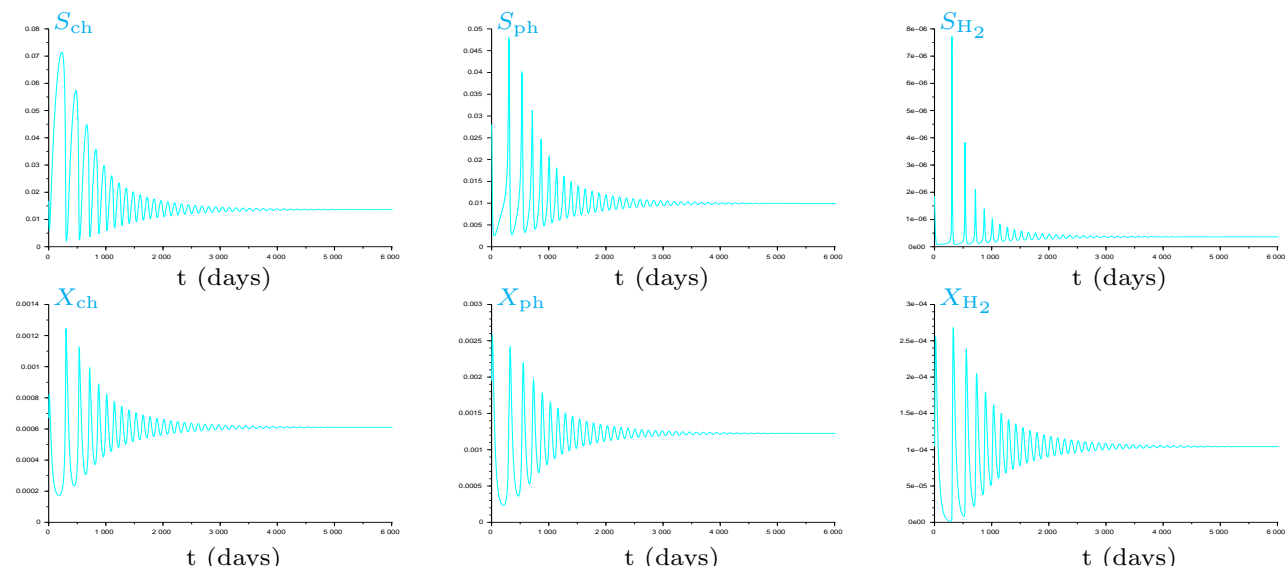

$\mathrm{t}$ (days)

$\mathrm{t}$ (days)

FIG. H.3. Trajectories of $S_{\mathrm{ch}}, S_{\mathrm{ph}}, S_{\mathrm{H}_{2}}, X_{\mathrm{ch}}, X_{\mathrm{ph}}$ and $X_{\mathrm{H}_{2}}$ for $\mathrm{S}_{\mathrm{ch}, \mathrm{in}}=0.11\left(\right.$ in $\left.\mathrm{kgCOD} / \mathrm{m}^{3}\right)$ : Convergence to the positive steady state $\mathrm{SS} 6$.
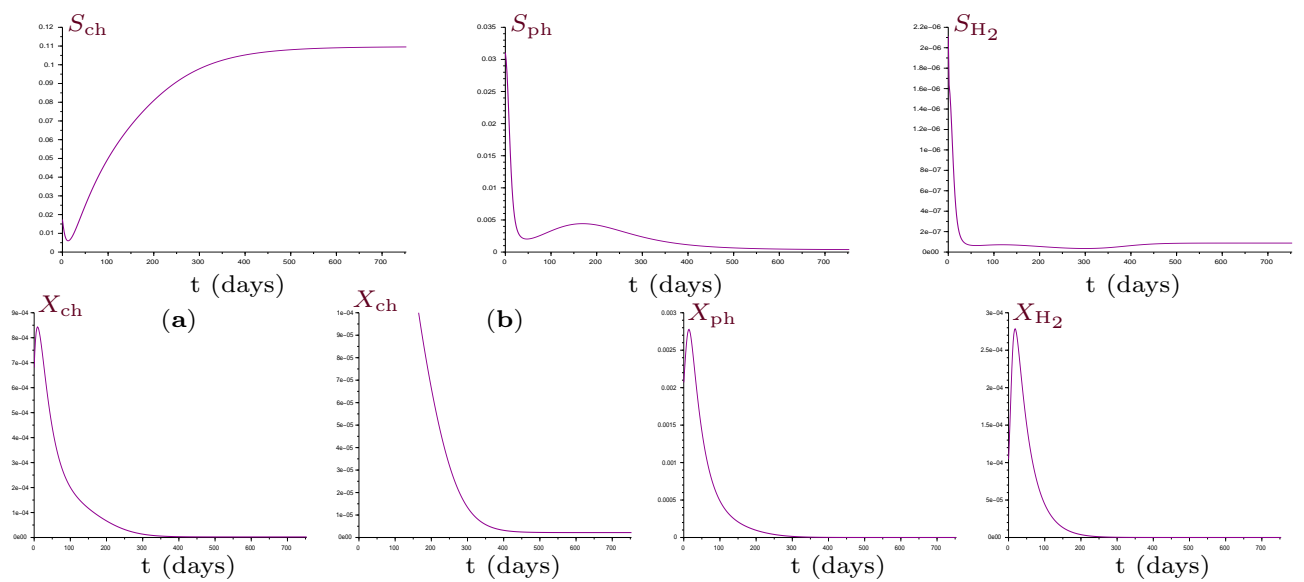

FIG. H.4. Trajectories of $S_{\mathrm{ch}}, S_{\mathrm{ph}}, S_{\mathrm{H}_{2}}, X_{\mathrm{ch}}, X_{\mathrm{ph}}$ and $X_{\mathrm{H}_{2}}$ for $\mathrm{S}_{\mathrm{ch}, \text { in }}=0.11$ (in $\left.\mathrm{kgCOD} / \mathrm{m}^{3}\right)$ : Convergence to the stable steady state SS3. (b) A magnification of (a) showing that the solution of (4.2) converges to the nonzero $X_{\mathrm{ch}}$-component of SS3.
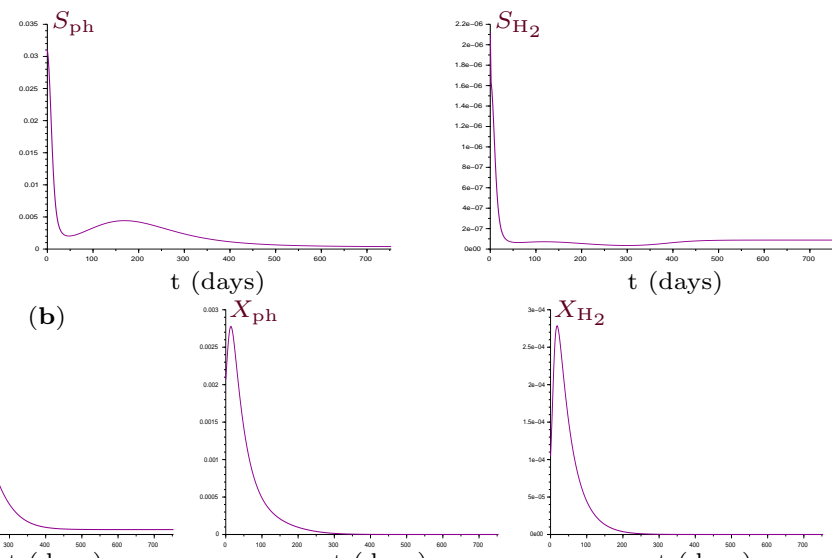

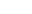

[1] D. Batstone, J. Keller, I. Angelidaki, S. Kalyuzhnyi, S. Pavlostathis, A. Rozzi, W. Sanders, H. Siegrist, and V. Vavilin, The IWA Anaerobic Digestion Model No 1 (ADM1), Water Sci Technol., 45 (2002), pp. 65-73, https://doi.org/10.2166/wst.2002.0292.

[2] B. Benyahia, T. Sari, B. Cherki, and J. Harmand, Bifurcation and stability analysis of a two step model for monitoring anaerobic digestion processes, J. Proc. Control, 22 (2012), pp. 10081019, https://doi.org/10.1016/j.jprocont.2012.04.012.

[3] O. Bernard, Z. Hadj-Sadok, D. Dochain, A. Genovesi, and J.-P. Steyer, Dynamical model development and parameter identification for an anaerobic wastewater treatment process, Biotechnol. Bioeng., 75 (2001), pp. 424-438, https://doi.org/10.1002/bit.10036.

[4] A. Bornhöft, R. Hanke-Rauschenbach, and K. Sundmacher, Steady-state analysis of the Anaerobic Digestion Model No. 1 (ADM1), J. Nonlinear Dyn., 73 (2013), pp. 535-549, https://doi. org/10.1007/s11071-013-0807-x.

[5] A. Burchard, Substrate degradation by a mutualistic association of two species in the chemostat, J. Math. Biol., 32 (1994), pp. 465-489, https://doi.org/10.1007/BF00160169.

[6] W. A. Coppel, Stability and Asymptotic Behavior of Differential Equations, D.C. Heath, Boston, 1965. 
TABLE 13

Steady states SS3 and SS6 of model (4.2) corresponding to Figures 4.2 to 4.4 and H.1 to H.4. The biological parameters are provided in Table 15 . The operating parameters are $D=0.01, S_{\mathrm{ph}}^{\mathrm{in}}=0$, $S_{\mathrm{H}_{2}}^{\mathrm{in}}=2.67 \times 10^{-5}$ and $S_{\mathrm{ch}}^{\mathrm{in}}$ given in the second column.

\begin{tabular}{lll} 
Figure & $S_{\mathrm{ch}}^{\mathrm{in}}$ & $\begin{array}{l}\mathrm{SS} 3=\left(X_{\mathrm{ch}}, 0,0, S_{\mathrm{ch}}, S_{\mathrm{ph}}, S_{\mathrm{H}_{2}}\right) \\
\mathrm{SS} 6=\left(X_{\mathrm{ch}}, X_{\mathrm{ph}}, X_{\mathrm{H}_{2}}, S_{\mathrm{ch}}, S_{\mathrm{ph}}, S_{\mathrm{H}_{2}}\right)\end{array}$ \\
\hline 4.2 & \multirow{2}{*}{0.098} & $\begin{array}{l}\left(2.1910^{-6}, 0,0,9.7710^{-2}, 3.6510^{-4}, 9.1710^{-8}\right) \\
\left(5.3410^{-4}, 1.0610^{-3}, 8.8010^{-5}, 1.3610^{-2}, 9.9310^{-3}, 3.6210^{-7}\right)\end{array}$ \\
\hline 4.3 & & $\left(2.1910^{-6}, 0,0,9.9210^{-2}, 3.6510^{-4}, 9.1210^{-8}\right)$ \\
H.1 & 0.0995 & $\left(5.4410^{-4}, 1.0810^{-3}, 9.0010^{-5}, 1.3610^{-2}, 9.9310^{-3}, 3.6210^{-7}\right)$ \\
H.2 & & \\
\hline 4.4 & & $\left(2.1910^{-6}, 0,0,1.1010^{-1}, 3.6510^{-4}, 8.7910^{-8}\right)$ \\
H.3 & 0.11 & $\left(6.1010^{-4}, 1.2210^{-3}, 1.0410^{-4}, 1.3610^{-2}, 9.9310^{-3}, 3.6210^{-7}\right)$ \\
H.4 & &
\end{tabular}

TABLE 14

The initial conditions of solutions of model (4.2) in Figures 4.2 to 4.4 and H.1 to H.4 are obtained from the initial conditions of the solutions of model (H.2) by using the change of variables (H.1). The initial conditions of (H.2) are given by $X_{i}(0)=X_{i}^{*}+\varepsilon$ and $S_{i}(0)=S_{i}^{*}+\varepsilon, i=0,1,2$ where $X_{i}^{*}$ and $S_{i}^{*}$ are the components of SS6 and $\varepsilon$ is given in the second column. When there is more than one trajectory in the figure, its color is indicated in the first column.

\begin{tabular}{lll}
$\begin{array}{l}\text { Figure } \\
\text { Color }\end{array}$ & $\varepsilon$ & $\left(X_{\mathrm{ch}}(0), X_{\mathrm{ph}}(0), X_{\mathrm{H}_{2}}(0), S_{\mathrm{ch}}(0), S_{\mathrm{ph}}(0), S_{\mathrm{H}_{2}}(0)\right)$ \\
\hline 4.2 & $9.710^{-3}$ & $\left(5.4410^{-4}, 1.1710^{-3}, 8.8010^{-5}, 1.4210^{-2}, 1.2910^{-2}, 6.0510^{-7}\right)$ \\
\hline 4.3 & & \\
Pink & $10^{-2}$ & $\left(5.5410^{-4}, 1.2010^{-3}, 9.0010^{-5}, 1.4210^{-2}, 1.2910^{-2}, 6.1210^{-7}\right)$ \\
Blue & $3.210^{-2}$ & $\left(5.7610^{-4}, 1.4610^{-3}, 9.0010^{-5}, 1.5310^{-2}, 1.9610^{-2}, 1.1610^{-6}\right)$ \\
Green & $3.510^{-2}$ & $\left(5.7910^{-4}, 1.5010^{-3}, 9.0010^{-5}, 1.5510^{-2}, 2.0510^{-2}, 1.2410^{-6}\right)$ \\
\hline 4.4 & & \\
Blue & $610^{-2}$ & $\left(6.7110^{-4}, 1.9510^{-3}, 1.0410^{-4}, 1.6810^{-2}, 2.8010^{-2}, 1.8610^{-6}\right)$ \\
Green & $710^{-2}$ & $\left(6.8110^{-4}, 2.0710^{-3}, 1.0410^{-4}, 1.7410^{-2}, 3.1110^{-2}, 2.1110^{-6}\right)$ \\
\hline H.1 & $210^{-3}$ & $\left(5.4610^{-4}, 1.1010^{-3}, 9.0010^{-5}, 1.3710^{-2}, 1.0510^{-2}, 4.1210^{-7}\right)$ \\
H.2 & $3.510^{-2}$ & $\left(5.7910^{-4}, 1.5010^{-3}, 9.0010^{-5}, 1.5510^{-2}, 2.0510^{-2}, 1.2410^{-6}\right)$ \\
H.3 & $610^{-2}$ & $\left(6.7110^{-4}, 1.9510^{-3}, 1.0410^{-4}, 1.6810^{-2}, 2.8010^{-2}, 1.8610^{-6}\right)$ \\
H.4 & $710^{-2}$ & $\left(6.8110^{-4}, 2.0710^{-3}, 1.0410^{-4}, 1.7410^{-2}, 3.1110^{-2}, 2.1110^{-6}\right)$
\end{tabular}

[7] Y. Daoud, N. Abdellatif, T. Sari, and J. Harmand, Steady state analysis of a syntrophic model: The effect of a new input substrate concentration, Math. Model. Nat. Phenom., 13 (2018), pp. 1-22, https://doi.org/10.1051/mmnp/2018037.

[8] M. El Hajji, N. Chorfi, and M. Jleli, Mathematical modelling and analysis for a three-tiered microbial food web in a chemostat, Electron. J. Differ. Equ., 255 (2017), pp. 1-13.

[9] M. El Hajji, F. Mazenc, and J. Harmand, A mathematical study of a syntrophic relationship of a model of anaerobic digestion process, Math. Biosci. Eng., 7 (2010), pp. 641-656, https://doi.org/10.3934/mbe.2010.7.641.

[10] F. Gantmacher, Application of the theory of matrices, Interscience Publishers, INC. New York, 2004.

[11] MAPLE, version 17.0.0.0, Waterloo Maple Inc., Waterloo, Ontario, 2018.

[12] C. Martinez, A. Ávila, F. Mairet, L. Meier, and D. Jeison, Modeling and analysis of an absorption column connected to a microalgae culture, SIAM J. Appl. Math., 80 (2020), pp. 772-791, https://doi.org/10.1137/18M1225641.

[13] J. Mata-Alvarez, S. Macè, and P. Llabrès, Anaerobic digestion of organic solid wastes. an overview of research achievements and perspectives, Bioresour. Technol., 74 (2000), pp. 3-16, https: 
TABLE 15

Nominal parameter values, where $i=\mathrm{ch}, \mathrm{ph}, \mathrm{H}_{2}$. Units are expressed in Chemical Oxygen Demand (COD).

\begin{tabular}{lll} 
Parameters & Nominal values & Units \\
\hline$k_{m, \mathrm{ch}}$ & 29 & $\mathrm{kgCOD} / \mathrm{kgCOD}_{\mathrm{X}} / \mathrm{d}$ \\
$K_{S, \mathrm{ch}}$ & 0.053 & $\mathrm{kgCOD} / \mathrm{m}^{3}$ \\
$Y_{\mathrm{ch}}$ & 0.019 & $\mathrm{kgCOD} \times$ \\
$k_{m, \mathrm{ph}}$ & 26 & $\mathrm{kgCOD}$ \\
$K_{S, \mathrm{ph}}$ & 0.302 & $\mathrm{kgCOD}$ \\
$Y_{\mathrm{ph}} / \mathrm{kgCOD}_{\mathrm{X}} / \mathrm{d}$ \\
$k_{m, \mathrm{H}_{2}}$ & 0.04 & $\mathrm{kgCOD} / \mathrm{m}^{3}$ \\
$K_{S, \mathrm{H}_{2}}$ & 35 & $\mathrm{kgCOD}$ \\
$K_{S, \mathrm{H}_{2}, \mathrm{c}}$ & $2.5 \times 10^{-5}$ & $\mathrm{kgCOD} / \mathrm{m}^{3}$ \\
$Y_{\mathrm{H}_{2}}$ & $0.0 \times 10^{-6}$ & $\mathrm{kgCOD} / \mathrm{m}^{3}$ \\
$k_{\mathrm{dec}, \mathrm{i}}$ & 0.02 & $\mathrm{kgCOD}$ \\
$K_{I, \mathrm{H}_{2}}$ & $3.5 \times 10^{-6}$ & $\mathrm{kgCOD}_{\mathrm{S}}$ \\
& & $\mathrm{kgCOD} / \mathrm{m}^{3}$
\end{tabular}

TABLE 16

Auxiliary functions in the case of growth functions given by (G.3).

Auxiliary function

Definition domain

$\begin{array}{ll}M_{0}\left(y, s_{2}\right)=\frac{y K_{0}\left(L_{0}+s_{2}\right)}{m_{0} s_{2}-y\left(L_{0}+s_{2}\right)} & 0 \leq y<\frac{m_{0} s_{2}}{L_{0}+s_{2}} \\ M_{1}\left(y, s_{2}\right)=\frac{y K_{1}\left(K_{I}+s_{2}\right)}{m_{1} K_{I}-y\left(K_{I}+s_{2}\right)} & 0 \leq y<\frac{m_{1} K_{I}}{K_{I}+s_{2}} \\ M_{2}(y)=\frac{y K_{2}}{m_{2}-y} & 0 \leq y<m_{2} \\ M_{3}\left(s_{0}, z\right)=\frac{z L_{0}\left(K_{0}+s_{0}\right)}{m_{0} s_{0}-z\left(K_{0}+s_{0}\right)} & 0 \leq z<\frac{m_{0} s_{0}}{K_{0}+s_{0}} \\ s_{2}^{0}(D)=\frac{L_{0}\left(D+a_{0}\right)}{m_{0}-D-a_{0}} & D+a_{0}<m_{0} \\ s_{2}^{1}(D)=\frac{K_{I}\left(m_{1}-D-a_{1}\right)}{D+a_{1}} & D+a_{1}<m_{1} \\ \Psi\left(s_{2}, D\right)=(1-\omega) \frac{\left(D+a_{0}\right) K_{0}\left(L_{0}+s_{2}\right)}{m_{0} s_{2}-\left(D+a_{0}\right)\left(L_{0}+s_{2}\right)} & \left\{D \in I_{1}: s_{2}^{0}<s_{2}<s_{2}^{1}\right\} \\ \quad+\frac{\left(D+a_{1}\right) K_{1}\left(K_{I}+s_{2}\right)}{m_{1} K_{I}-\left(D+a_{1}\right)\left(K_{I}+s_{2}\right)}+s_{2} & \\ \psi_{0}\left(s_{0}\right)=\frac{m_{0} s_{0}\left(s_{2}^{\text {in }}-\omega\left(s_{0}^{\text {in }}-s_{0}\right)\right)}{\left(K_{0}+s_{0}\right)\left(L_{0}+s_{2}^{\text {in }}-\omega\left(s_{0}^{\text {in }}-s_{0}\right)\right)} & s_{0} \in\left[\max \left(0, s_{0}^{\text {in }}-s_{2}^{\text {in }} / \omega\right),+\infty\right) \\ \psi_{1}\left(s_{1}\right)=\frac{m_{1} s_{1} K_{I}}{\left(K_{1}+s_{1}\right)\left(K_{I}+s_{2}^{\text {in }}+s_{1}^{\text {in }}-s_{1}\right)} & s_{1} \in\left[0, s_{1}^{\text {in }}+s_{2}^{\text {in }}\right]\end{array}$

//doi.org/10.1016/S0960-8524(00)00023-7.

[14] R. May, Stability and Complexity in Model Ecosystems, Princeton Univ. Press, NJ., USA, 1973.

[15] T. Meadows, M. Weedermann, and G. Wolkowicz, Global analysis of a simplified model of anaerobic digestion and a new result for the chemostat, SIAM J. Appl. Math., 79 (2019), pp. 668689, https://doi.org/10.1137/18M1198788.

[16] S. Nouaoura, R. Fekih-Salem, N. Abdellatif, and T. Sari, Mathematical analysis of a three-tiered food-web in the chemostat, Discrete \& Continuous Dyn. Syst. - B, (2020), https://doi.org/ 10.3934/dcdsb.2020369.

[17] P. J. Reilly, Stability of commensalistic systems, Biotechnol. Bioeng., 16 (1974), pp. 1373-1392, https://doi.org/10.1002/bit.260161006.

[18] T. Sari, M. El Hajji, and J. Harmand, The mathematical analysis of a syntrophic relationship between two microbial species in a chemostat, Math. Biosci. Eng., 9 (2012), pp. 627-645, https://doi.org/10.3934/mbe.2012.9.627.

[19] T. Sari and J. Harmand, A model of a syntrophic relationship between two microbial species in a chemostat including maintenance, Math. Biosci., 275 (2016), pp. 1-9, https://doi.org/ 10.1016/j.mbs.2016.02.008.

[20] T. Sari and M. J. Wade, Generalised approach to modelling a three-tiered microbial food-web, Math. Biosci., 291 (2017), pp. 21-37, https://doi.org/10.1016/j.mbs.2017.07.005. 
[21] M. Sbarciog, M. Loccufier, and E. Noldus, Determination of appropriate operating strategies for anaerobic digestion systems, Biochem. Eng. J., 51 (2010), pp. 180-188, https://doi.org/ 10.1016/j.bej.2010.06.016.

[22] SCILAB, version 6.0.1(64-bit), Scilab Enterprises SAS, 2018.

[23] S. Sobieszek, M. J. Wade, and G. S. K. Wolkowicz, Rich dynamics of a three-tiered anaerobic food-web in a chemostat with multiple substrate inflow, Math. Biosci. Eng., 17 (2020), pp. 7045-7073, https://doi.org/10.3934/mbe.2020363.

[24] E. I. P. Volcke, M. Sbarciog, E. J. L. Noldus, B. De Baets, and M. Loccufier, Steady state multiplicity of two-step biological conversion systems with general kinetics, Math. Biosci., 228 (2010), pp. 160-170, https://doi.org/10.1016/j.mbs.2010.09.004.

[25] M. Wade, Not just numbers: Mathematical modelling and its contribution to anaerobic digestion processes, Processes, 8 (2020), p. 888, https://doi.org/10.3390/pr8080888.

[26] M. J. Wade, R. W. Pattinson, N. G. Parker, and J. Dolfing, Emergent behaviour in a chlorophenolmineralising three-tiered microbial 'food web', J. Theoret. Biol., 389 (2016), pp. 171-186, https://doi.org/10.1016/j.jtbi.2015.10.032.

[27] M. Weedermann, G. Seo, and G. S. K. Wolkowicz, Mathematical model of anaerobic digestion in a chemostat: Effects of syntrophy and inhibition, J. Biol. Dyn., 7 (2013), pp. 59-85, https://doi.org/10.1080/17513758.2012.755573.

[28] M. Weedermann, G. S. K. Wolkowicz, and J. Sasara, Optimal biogas production in a model for anaerobic digestion, J. Nonlinear Dyn., 81 (2015), pp. 1097-1112, https://doi.org/10.1007/ s11071-015-2051-z.

[29] A. Xu, J. Dolfing, T. P. Curtis, G. Montague, and E. Martin, Maintenance affects the stability of a two-tiered microbial 'food chain'?, J. Theoret. Biol., 276 (2011), pp. 35-41, https: //doi.org/10.1016/j.jtbi.2011.01.026. 